\title{
ON FACTORIZATION ALGEBRAS ARISING IN THE QUANTUM GEOMETRIC LANGLANDS THEORY
}

\author{
D. GAITSGORY
}

\begin{abstract}
We study factorization algebras on configuration spaces of points on a curve, colored by elements of the root lattice. Our main result says that the factorization algebra attached to Lusztig's quantum group can be obtained as a direct image of a twisted Whittaker sheaf on the Zastava space.
\end{abstract}

\section{Contents}

Introduction

0.1. Framework for this paper: the FLE

0.2 . What is actually done in this paper?

0.3. Strategy of proof: quantum groups creep in

0.4. Organization of the paper

0.5. Notations and conventions

0.6. Acknowledgements

1. Factorization algebras attached to quadratic forms

1.1. The parameters

1.2. The configuration space

1.3. Gerbes on the configuration space

1.4. Factorization algebras

1.5. The factorization algebra on the open part

1.6. Construction of the "small" factorization algebra $\Omega_{q}^{\mathrm{sml}}$

1.7. Construction of the DeConcini-Kac and Lusztig versions

2. Properties of the DeConcini-Kac version

2.1. First properties of $\Omega_{q}^{\mathrm{DK}}$

2.2. Properties of $\Omega_{q}^{\mathrm{DK}}$ in characteristic 0

2.3. Inductive construction of $\Omega_{q}^{\mathrm{DK}}$

2.4. Further properties of $\Omega_{q}^{\mathrm{DK}}$ in characteristic 0

2.5. A sharper estimate

3. Factorization algebras via quantum groups

3.1. Quantum Hopf algebras

3.2. The free, cofree and small versions of the positive part of the quantum group

3.3. The DeConcini-Kac and Luztig's versions of the positive part of the quantum group

3.4. From Hopf algebras to factorization algebras

3.5. Factorization algebras attached to quantum groups

3.6. The first comparison: $\Omega_{q, \text { Quant }}^{\mathrm{KD}}$ vs abstract $\Omega_{q}^{\mathrm{KD}}$

3.7. Proof of the properties of $\Omega_{q}^{\mathrm{KD}}$ in characteristic 0

4. The quantum Frobenius

4.1. The quantum Frobenius lattice

4.2. Convolution factorization algebras

4.3. The classical factorization algebra $\Omega^{\sharp, c l}$

4.4. Relationship of $\Omega_{q}^{\mathrm{Lus}}$ with $\Omega_{q}^{\mathrm{sml}}$ via $\Omega^{\mathrm{cl}}$ 
4.5. The quantum Frobenius map for quantum groups 45

4.6. Quantum Frobenius for factorization algebras $\quad 48$

5. Zastava spaces and Whittaker/semi-infinite categories $\quad 48$

5.1. Affine Grassmannian over the configuration space $\quad 48$

5.2. Semi-infinite orbits and their closures $\quad 49$

5.3. Zastava spaces $\quad 52$

5.4. Sheaves on Zastava spaces $\quad 53$

\begin{tabular}{ll|} 
5.5. The Whittaker category & 56 \\
\hline 5.6.
\end{tabular}

5.6. The semi-infinite category $\quad 57$

5.7. Gauss objects via the Whittaker and the semi-infinite category $\quad 60$

6. Factorization algebras arising from the Whittaker sheaf $\quad 61$

6.1. Factorization algebras $\Omega_{q, \text { Whit }}^{\mathrm{sml}}, \Omega_{q, \text { Whit }}^{\mathrm{Lus}}$ and $\Omega_{q, \text { Whit }}^{\mathrm{DK}} \quad 61$

6.2. Statement of the main results $\quad 62$

\begin{tabular}{lll|}
6.3. & Interpretation as sub-top cohomology vanishing & 63 \\
\hline
\end{tabular}

6.4. The non-torsion valued case $\quad 65$

6.5. Adding the formal parameter $\quad 65$

6.6. Quantum Frobenius for $\Omega_{q, \text { Whit }}^{\text {Lus }} \quad 67$

6.7. Geometric construction of the quantum Frobenius $\quad 68$

\begin{tabular}{lll}
7. & Geometry and combinatorics of Theorem 6.3.8 & 69 \\
\hline
\end{tabular}

7.1. The goal $\quad 69$

\begin{tabular}{llr|} 
7.2. & Scrutinizing irreducible components & 69 \\
\hline
\end{tabular}

\begin{tabular}{ll} 
7.3. & The suspects \\
\hline 7.4.
\end{tabular}

\begin{tabular}{ll} 
7.4. Interlude: coordinates on the irreducible components & 72 \\
\hline
\end{tabular}

\begin{tabular}{ll}
7.5. & 75 \\
\hline 7.6. & Indictment
\end{tabular}

\begin{tabular}{ll} 
7.6. Conviction & 76 \\
\hline 7.7.
\end{tabular}

7.7. The verdict $\quad 77$

Appendix A. Sheaves with a formal parameter $\quad 77$

A.1. Digression: sheaves with a formal parameter $\quad 77$

$\begin{array}{lll}\text { A.2. The t-structure } & 78\end{array}$

\begin{tabular}{lll} 
A.3. Gerbes with a formal parameter & 79 \\
\hline
\end{tabular}

References

\section{INTRODUCTION}

0.1. Framework for this paper: the FLE. This paper is a step towards the proof of the Fundamental Local Equivalence (FLE), which is a (still conjectura 1]) equivalence between the twisted Whittaker category on the affine Grassmannian of a reductive group $G$ and the Kazhdan-Lusztig category of its Langlands dual $\check{G}$.

In this subsection we will recall what FLE is and what role this paper plays in our strategy to prove it.

0.1.1. We start with a datum of level for $G$, which is an $\operatorname{Ad}_{G}$-invariant symmetric bilinear form $\kappa$ on the Lie algebra $\mathfrak{g}$ of $G$. Given $\kappa$, we can consider the twisted category of D-modules D- $\bmod _{\kappa}\left(\mathrm{Gr}_{G}\right)$ on the affine Grassmannian $\operatorname{Gr}_{G}=G((t)) / G \llbracket t \rrbracket$ of $G$, and the subcategory

$$
\text { Whit }_{\kappa}\left(\mathrm{Gr}_{G}\right) \subset \mathrm{D}-\bmod _{\kappa}\left(\mathrm{Gr}_{G}\right) \text {, }
$$

obtained by imposing the condition of $N((t))$-equivariance against a non-degenerate character.

Suppose now that $\kappa$ is such that the form

$$
\kappa+\left.\frac{\kappa_{\text {Kil }}}{2}\right|_{\mathfrak{t}}
$$

\footnotetext{
${ }^{1}$ Recently, a proof by methods different than what we envisaged, was obtained by J. Campbell, G. Dhillon and S. Raskin.
} 
on the Cartan subalgebra $\mathfrak{t} \subset \mathfrak{g}$ is non-degenerate. Then to $\kappa$ one can attach its dual level for $\check{G}$, denoted $\check{\kappa}$, so that the forms

$$
\kappa+\left.\frac{\kappa_{\mathrm{Kil}}}{2}\right|_{\mathfrak{t}} \text { and } \check{\kappa}+\left.\frac{\check{\kappa}_{\mathrm{Kil}}}{2}\right|_{\check{\mathfrak{t}}}
$$

are each other's duals under the identification

$$
\check{\mathfrak{t}} \simeq \mathfrak{t}^{\vee} .
$$

We will consider the affine Kac-Moody Lie algebra

$$
0 \rightarrow k \rightarrow \widehat{\hat{\mathfrak{g}}}_{\check{\kappa}} \rightarrow \check{\mathfrak{g}}((t)) \rightarrow 0
$$

attached to $\check{\mathfrak{g}}$ and $\check{\kappa}$ (here $k$ is the ground field), and the category, denoted $\operatorname{KL}_{\check{\kappa}}(\check{G})$, of representations of the Harish-Chandra pair $\left(\widehat{\mathfrak{g}}_{\check{\kappa}}, \check{G} \llbracket t \rrbracket\right)$.

The Fundamental Local Equivalence says that there exists a (canonically defined) equivalence of categories

$$
\operatorname{Whit}_{\kappa}\left(\operatorname{Gr}_{G}\right) \simeq \operatorname{KL}_{\check{\kappa}}(\check{G}) \text {. }
$$

The equivalence (0.2) is not an easy statement to prove because it involves two geometrically defined categories, one for $G$ and another for $\check{G}$, while the relationship between $G$ and $\check{G}$ is combinatorial in nature (involution on the root data). Our method of attacking the FLE consists of expressing both sides in terms of objects that are combinatorially attached to the root datum and then comparing these directly.

0.1.2. The point of departure is that when $G$ is a torus $T$, the corresponding equivalence

$$
\operatorname{Whit}_{\kappa}\left(\operatorname{Gr}_{T}\right) \simeq \operatorname{KL}_{\check{\kappa}}(\check{T}),
$$

is relatively easy to construct (note that for a torus Whit ${ }_{\kappa}\left(\operatorname{Gr}_{T}\right)=\mathrm{D}-\bmod _{\kappa}\left(\operatorname{Gr}_{T}\right)$ ).

Hence, the natural desire is to describe Whit ${ }_{\kappa}\left(\operatorname{Gr}_{G}\right)$ (resp., $\mathrm{KL}_{\breve{\kappa}}(\check{G})$ ) in terms of Whit ${ }_{\kappa}\left(\mathrm{Gr}_{T}\right)$ (resp., $\left.\mathrm{KL}_{\check{\kappa}}(\check{T})\right)$ and to show that these descriptions match up under the equivalence (0.3).

Next comes the crucial observation that the two sides of (0.2) are not just plain (DG) categories; rather they naturally extend to factorization categories over any smooth curve (we are thinking of the variable $t$ in $k \llbracket t \rrbracket \subset k((t))$ as a coordinate at a point $x$ on a curve $X)$. Moreover, the conjectural equivalence (0.2) is supposed to be an equivalence as factorization categories. In particular, the equivalence in (0.3) does have this structure.

The categories Whit ${ }_{\kappa}\left(\mathrm{Gr}_{G}\right)$ and $\mathrm{KL}_{\check{\kappa}}(\check{G})$ are equipped with naturally defined Jacquet functors

$$
J_{\text {Whit }}: \text { Whit }_{\kappa}\left(\operatorname{Gr}_{G}\right) \rightarrow \text { Whit }_{\kappa}\left(\operatorname{Gr}_{T}\right) \text { and } J_{\mathrm{KL}}: \operatorname{KL}_{\check{\kappa}}(\check{G}) \rightarrow \mathrm{KL}_{\check{\kappa}}(\check{T})
$$

and each of these functors has a naturally defined factorization structure.

Define

$$
\Omega_{\kappa, \text { Whit }}:=J_{\text {Whit }}\left(\mathbf{1}_{\text {Whit }_{\kappa}\left(\operatorname{Gr}_{G}\right)}\right) \in \text { Whit }_{\kappa}\left(\operatorname{Gr}_{T}\right) \text { and } \Omega_{\check{\kappa}, \mathrm{KL}}:=J_{\mathrm{KL}}\left(\mathbf{1}_{\mathrm{KL}_{\check{\kappa}}(\check{G})}\right) \in \operatorname{KL}_{\check{\kappa}}(\check{T}),
$$

where

$$
\mathbf{1}_{\mathrm{Whit}_{\kappa}\left(\mathrm{Gr}_{G}\right)} \in \mathrm{Whit}_{\kappa}\left(\mathrm{Gr}_{T}\right) \text { and } \mathbf{1}_{\mathrm{KL}_{\check{\kappa}}(\check{G})} \in \mathrm{KL}_{\check{\kappa}}(\check{T})
$$

are the unit (a.k.a. vacuum) objects.

The factorization structure on the functor $J_{\text {Whit }}$ (resp., $J_{\mathrm{KL}}$ ) defines on $\Omega_{\kappa}$,Whit $\left(\right.$ resp., $\Omega_{\check{\kappa}, \mathrm{KL}}$ ) a structure of factorization algebra in the corresponding factorization category, i.e., Whit ${ }_{\kappa}\left(\operatorname{Gr}_{T}\right)($ resp., $\mathrm{KL}_{\check{\kappa}}(\check{T})$ ). Furthermore, the functors $J_{\text {Whit }}$ and $J_{\mathrm{KL}}$ upgrade to functors

$$
J_{\text {Whit }}^{\text {enh }}: \text { Whit }_{\kappa}\left(\operatorname{Gr}_{G}\right) \rightarrow \Omega_{\kappa, \text { Whit }}-\text { FactMod }\left(\text { Whit }_{\kappa}\left(\operatorname{Gr}_{T}\right)\right)
$$

and

$$
J_{\mathrm{KL}}^{\mathrm{enh}}: \mathrm{KL}_{\check{\kappa}}(\check{G}) \rightarrow \Omega_{\check{\kappa}, \mathrm{KL}}-\operatorname{FactMod}\left(\mathrm{KL}_{\check{\kappa}}(\check{T})\right),
$$

respectively, where the notation

$$
\mathcal{A} \text {-FactMod(e) }
$$


stands for the category of factorization modules for a factorization algebra $\mathcal{A}$ in a given factorization category $\mathcal{C}$.

In the best possible scenario, the functors (0.4) and (0.5) would be equivalences of categories. However, in our situation, they are not such. Yet, there exists an explicit procedure that allows to express the LHS in (0.4) (resp., (0.5) via the RHS, and we will discuss it in a subsequent publication.

Hence, for now we would like to match up the right-hand sides in (0.4) and (0.5). This amounts to constructing an isomorphism of factorization algebras

$$
\Omega_{\kappa, \text { Whit }} \simeq \Omega_{\check{\kappa}, \mathrm{KL}}
$$

with respect to the equivalence of factorization categories in 0.3 .

Remark 0.1.3. A similar strategy is supposed to lead to a new proof of the Kazhdan-Lusztig equivalence

$$
\operatorname{KL}_{\check{\kappa}}(\check{G}) \simeq \operatorname{Rep}_{q}(\check{G})
$$

where the latter is the category of modules over Lusztig's version of the quantum group, and $q$ is the quadratic form on the weight lattice of $\check{G}$ (=coweight lattice of $G$ ) with values in $\mathbb{C}^{\times}$obtained by exponentiating the form that we denote $q_{k}$ (here the ground field $k$ is $\mathbb{C}$ ), see Sect. 0.2 .2

The composite equivalence

$$
\text { Whit }_{\kappa}\left(\operatorname{Gr}_{G}\right) \simeq \operatorname{Rep}_{q}(\check{G})
$$

was the original form of the FLE suggested by J. Lurie and the author a number of years ago.

0.1.4. What we do in this paper is prove "a half" of (0.6). Right below we will explain what we mean by this in terms of the constructions Sect. 0.1 .2 (in Sect. 0.2 .1 we will translate this into the language adopted in the main body of the paper):

What we do is give an a priori construction of a certain factorization algebra in $\mathrm{Whit}_{\kappa}\left(\mathrm{Gr}_{T}\right)$, denote it $\Omega_{\kappa}$, and show that it is isomorphic to $\Omega_{\kappa}$,Whit .

The main feature of $\Omega_{\kappa}$ is that it is combinatorial in nature, in that its construction only involves the coweight lattice $\Lambda$ of $G$ and the form (0.1).

In a subsequent publication we will establish an isomorphism

$$
\Omega_{\kappa} \simeq \Omega_{\check{\kappa}, \mathrm{KL}}
$$

That would be the "second half" of (0.6).

\section{2 . What is actually done in this paper?}

0.2.1. We will now describe the passage from what we just explained in Sect. 0.1.4 to the actual contents of the paper.

We observe that the factorization algebra $\Omega_{\kappa}$, Whit belongs to the full factorization subcategory of Whit ${ }_{\kappa}\left(\mathrm{Gr}_{T}\right)$ where we restrict the coweights of $T$ to belong to $\Lambda^{\text {neg }} \subset \Lambda$ (i.e., linear combinations of positive simple coroots with non-positive integral coefficients) 2 .

Now, by GLys2, 4.6], the category of factorization algebras within this subcategory can be identified with the category of factorization algebras in the category

$$
\mathrm{D}-\bmod _{\mathcal{G}_{\kappa} \text { Conf }}(\text { Conf })
$$

where Conf is the configuration space of points on $X$ colored by negative coweights (see Sect. 1.2) and $\mathcal{G}_{\kappa}^{\text {Conf }}$ is a (factorization) gerbe on Conf attached to the form (0.1), see Sect. 1.3.

So, in the main body of the paper we:

- Construct explicitly a factorization algebra $\Omega_{\kappa}$ in $D-\bmod _{\mathcal{G}_{\kappa} \text { Conf }}($ Conf $)$, see Sect. 1.7 .

- Construct the factorization algebra $\Omega_{\kappa}$,Whit in $\mathrm{D}-\bmod _{\mathcal{G} \text { Conf }}(\mathrm{Conf})$, using the geometry of Whittaker sheaves on the affine Grassmannian, see Sect. 6.1]

\footnotetext{
${ }^{2}$ Technically, we also observe that the coweight 0 component of $\Omega_{\kappa}$,Whit is the unit $\mathbf{1}_{\mathrm{Whit}}\left(\mathrm{Gr}_{T}\right)$, so $\Omega_{\kappa, \mathrm{Whit}}$ is naturally augmented, and we will actually work with its augmentation ideal rather than $\Omega_{\kappa}$, Whit itself.
} 
- Establish a (canonical) isomorphism of factorization algebras

$$
\Omega_{\kappa, \text { Whit }} \simeq \Omega_{\kappa}
$$

this is our main result, Theorem 6.2.5

Notational remark: In the main body of the paper, the above factorization algebras carry a superscript "Lus", to distinguish them from two other versions, which carry superscripts "DK" and "sml", respectively. All three versions are the same when $\kappa$ is irrational.

0.2.2. The advantage of interpreting $\Omega_{\kappa}$, whit as an object of $\mathrm{D}$ - $\bmod _{\mathcal{G}_{\kappa} \text { Conf }}($ Conf $)$ is that Conf is a scheme of finite type, so we find ourselves in the realm of usual algebraic geometry.

A crucial feature of the factorization algebras $\Omega_{\kappa}$ and $\Omega_{\kappa}$, whit is that, when viewed as objects of D- $\bmod _{\mathcal{G} \text { Conf }}(\mathrm{Conf})$, they are perverse, i.e., lie in the heart of the t-structure. It is this fact that will eventually allow us to construct an isomorphism between them.

We will now supply details as to how $\Omega_{\kappa}$ and $\Omega_{\kappa}$, whit are constructed and how the isomorphism (0.9) is established.

However, before we proceed any further, we make the following observation:

The two factorization algebras appearing in (0.9) are of geometric nature, i.e., they can be made sense of in a more general sheaf theory (see Sect. 0.5.4) and not just D-modules. Let explain what replaces the role of the form (0.1).

We note that we can interpret the form (0.1) as a symmetric bilinear form on $\Lambda$ with coefficients in $k$, denote it by $b_{k}$. Consider the quadratic form

$$
q_{k}(\lambda):=\frac{b_{k}(\lambda, \lambda)}{2}
$$

and let $q$ be the composition of $q_{k}$ with the projection $k \rightarrow k / \mathbb{Z}$. One can show that the gerbe $\mathcal{G}_{\kappa}^{\text {Conf }}$ only depends on the latter form $q$.

When we work with another sheaf theory

$$
Y \mapsto \operatorname{Shv}(Y),
$$

with a field of coefficients e, let $\mathfrak{Z}$ be the abelian group introduced in Sect. 0.5.6 i.e., elements $\zeta \in \mathfrak{Z}$ parameterize Kummer local systems on $\mathbb{G}_{m}$

$$
\zeta \mapsto \Psi_{\zeta}
$$

so for D-modules we have $\mathfrak{Z}=k / \mathbb{Z}$, and for constructible sheaves $\mathfrak{Z}=\mathrm{e}^{\times}$.

So for a general sheaf theory, our quantum parameter is a quadratic form

$$
q: \Lambda \rightarrow \mathfrak{Z}
$$

see Sect. 1.1.1 We denote the corresponding (factorization) gerbe on Conf by $\mathcal{G}_{q}^{\text {Conf }}$ (see Sect. 1.3), and the corresponding factorization algebras in $\operatorname{Shv}_{\mathcal{G}_{q}^{\text {Conf }}}($ Conf) will be denoted

$$
\Omega_{q}^{?} \text { and } \Omega_{q, \text { Whit }}^{?}
$$

where $?=$ Lus, DK, sml. 
0.2.3. We now explain how the factorization algebra $\Omega_{q}^{\mathrm{Lus}}$ is constructed.

The initial observation is that the gerbe $\mathcal{G}_{q}^{\text {Conf }}$ is canonically trivialized over the open subscheme

$$
\text { Conf } \stackrel{j}{\hookrightarrow} \text { Conf }
$$

consisting of simple divisors (pairwise distinct points of $X$ each carrying a negative simple coroot).

We let $\stackrel{\circ}{\Omega} \in \operatorname{Perv}($ Conf $)$ be the sign local system, which is the simplest kind of factorization algebra on

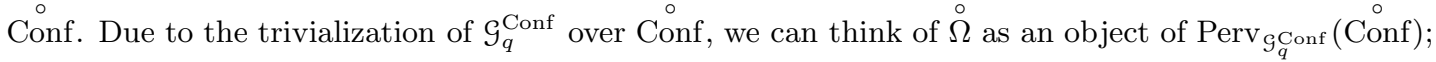
when thought of in this capacity we will denote it by $\stackrel{\circ}{\Omega}_{q}$.

For any value of $q$ we let $\Omega_{q}^{\text {sml }}$ denote the Goresky-MacPherson extension of $\stackrel{\circ}{\Omega}_{q}$ along the open embedding $j$ of (0.10). We emphasize that the above GM-extension is taking place in the category of $\mathcal{G}_{q}^{\text {Conf }}$-twisted sheaves (and not plain sheaves).

We now proceed to the definition of $\Omega_{q}^{\mathrm{DK}}$ and $\Omega_{q}^{\mathrm{Lus}}$.

When the values of $q$ in $\mathfrak{Z}$ are non-torsion we have

$$
\Omega_{q}^{\mathrm{DK}} \simeq \Omega_{q}^{\mathrm{sml}} \simeq \Omega_{q}^{\mathrm{Lus}}
$$

we denote the resulting factorization algebra simply by $\Omega_{q}$.

For $q$ that does take torsion values, we introduce a deformation of $q$ over a formal power series ring $\mathrm{e} \llbracket \hbar \rrbracket$, so that the image of $q$ over $\mathrm{e}((\hbar))$ is non-torsion valued. Hence, the factorization algebra $\Omega_{q_{((\hbar))}}$ is well-defined (see the paragraph above).

We set

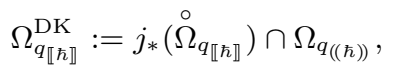

where the intersection is taking place in $j_{*}\left(\stackrel{\circ}{\Omega}_{q_{((\hbar))}}\right)$.

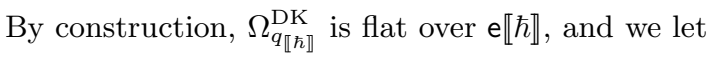

$$
\Omega_{q}^{\mathrm{DK}}:=\Omega_{q_{\llbracket \hbar \rrbracket}^{\mathrm{DK}}}^{\mathrm{e} \llbracket \hbar \rrbracket} \underset{\mathrm{e}}{\otimes} \in \operatorname{Perv}_{\mathcal{G}_{q}^{\text {Conf }}}(\text { Conf }) .
$$

Finally, we let $\Omega_{q}^{\mathrm{Lus}}$ be the Verdier dual of $\Omega_{q^{-1}}^{\mathrm{DK}}$, where we note that Verdier duality acts as an anti-equivalence from $\operatorname{Perv}_{\mathcal{G}_{q}^{\text {Conf }}}($ Conf $)$ to $\operatorname{Perv}_{\substack{\mathcal{G} \\ q^{-1}}}($ Conf $)$.

0.2.4. A remarkable feature of $\Omega_{q}^{\mathrm{Lus}}$ (and one that will ultimately allow us to prove the isomorphism with the Kac-Moody side, see (0.7) ) is that (under some mild assumption on the torsion, see Sect. 2.3.1) one can describe $\Omega_{q}^{\mathrm{Lus}}$ explicitly as a (twisted) perverse sheaf, see Sect. 2.3.8.

Namely, fix $\lambda \in \Lambda^{\text {neg }}$ and let Conf $^{\lambda}$ be the corresponding connected component of Conf (configurations of total degree $\lambda$ ). By induction and factorization, we can assume that $\left.\Omega_{q}^{\mathrm{Lus}}\right|_{\text {Conf }} \lambda$ has been defined away from the main diagonal

$$
X \stackrel{\Delta_{\lambda}}{\hookrightarrow} \operatorname{Conf}^{\lambda}, \quad x \mapsto \lambda \cdot x .
$$

Let $\jmath_{\lambda}$ be the embedding of the complementary open locus. Then:

- If $\lambda$ is of the form $w(\rho)-\rho$ with $\ell(w)=2$, we have

$$
\left.\Omega_{q}^{\text {Lus }}\right|_{\text {Conf } \lambda} \simeq\left(\jmath_{\lambda}\right){ }_{! *}\left(\left.\Omega_{q}^{\text {Lus }}\right|_{\text {Conf }^{\lambda}-\Delta_{\lambda}(X)}\right) ;
$$

- If $\lambda$ is not of the form $w(\rho)-\rho$ with $\ell(w)=2$, we have

$$
\left.\Omega_{q}^{\mathrm{Lus}}\right|_{\mathrm{Conf}^{\lambda}} \simeq H^{0}\left(\left(J_{\lambda}\right) !\left(\left.\Omega_{q}^{\mathrm{Lus}}\right|_{\operatorname{Conf}^{\lambda}-\Delta_{\lambda}(X)}\right)\right),
$$

where $H^{0}$ refers to taking 0th cohomology in the perverse t-structure (usual t-structure for D-modules). 
0.2.5. We now proceed to the definition of the factorization algebras

$$
\Omega_{q, \mathrm{Whit}}^{\mathrm{DK}}, \Omega_{q, \mathrm{Whit}}^{\mathrm{sml}}, \Omega_{q, \mathrm{Whit}}^{\mathrm{Lus}} .
$$

According to Sect. 0.1 .2 (adapted to our more general sheaf-theoretic setting), $\Omega_{q, \text { Whit }}^{\text {Lus }}$ is obtained by applying the functor

$$
J_{\text {Whit }}: \text { Whit }_{\mathcal{G}_{q}}\left(\mathrm{Gr}_{G}\right) \rightarrow \text { Whit }_{\mathcal{G}_{q}^{T}}\left(\mathrm{Gr}_{T}\right)
$$

to the unit object $\mathbf{1}_{\text {Whit }_{\mathcal{G}_{q}}\left(\mathrm{Gr}_{G}\right)} \in$ Whit $_{\mathcal{G}_{q}}\left(\mathrm{Gr}_{G}\right)$.

However, instead of going through all this (which would necessitate developing quite a bit of theory), we will write down directly what this construction produce 3 .

Let $z^{-}$be a version of the Zastava space, introduced in Sect. 5.3.3 Let

$$
\stackrel{\circ}{z} \stackrel{\mathbf{j}_{Z}^{-}}{\hookrightarrow} z^{-}
$$

be the open Zastava space; it is known to be smooth.

We have a natural projection

$$
\pi^{-}: z^{-} \rightarrow \text { Conf }
$$

and let $\stackrel{\circ}{\pi}$ denote its restriction to $\stackrel{\circ}{z}$.

A key feature of this situation is that the pullback of the gerbe $\mathcal{G}_{q}^{\text {Conf }}$ along $\stackrel{\circ}{\pi}$ acquires a canonical trivialization. Let

$$
\nabla_{q, Z}^{-}, \mathrm{IC}_{q, Z}^{-}, \Delta_{q, Z}^{-}
$$

be the $*_{-}$, GM- and !-extensions, respectively, of $\mathrm{IC}_{\stackrel{Z}{ }}$, but viewed as a $\mathcal{G}_{q}^{\text {Conf }}$-twisted sheaf.

There is a canonical map

$$
\chi: z^{-} \rightarrow \mathbb{G}_{a},
$$

given by the residue construction. Denote

$$
\operatorname{Gauss}_{q, *}^{-}:=\nabla_{q, Z}^{-} \stackrel{*}{\otimes} \chi^{*}(\exp ), \operatorname{Gauss}_{q, ! *}^{-}:=\mathrm{IC}_{q, Z}^{-} \stackrel{*}{\otimes} \chi^{*}(\exp ), \operatorname{Gauss}_{q, !}^{-}:=\Delta_{q, Z}^{-} \stackrel{*}{\otimes} \chi^{*}(\exp ) .
$$

All three are $\mathcal{G}_{q}^{\text {Conf }}$-twisted perverse sheaves on $Z^{-}$. Finally, set

$$
\Omega_{q, \mathrm{Whit}}^{\mathrm{DK}}:=\pi_{*}^{-}\left(\operatorname{Gauss}^{-} q, *\right), \Omega_{q, \mathrm{Whit}}^{\mathrm{sml}}:=\pi_{*}^{-}\left(\operatorname{Gauss}_{q, ! *}^{-}\right), \Omega_{q, \mathrm{Whit}}^{\mathrm{Lus}}:=\pi_{*}^{-}\left(\operatorname{Gauss}_{q, !}^{-}\right) .
$$

We note, however, that according to a cleanness result given by Theorem 5.4 .7 the maps

$$
\begin{aligned}
& \pi_{!}^{-}\left(\operatorname{Gauss}_{q, *}^{-}\right) \rightarrow \pi_{*}^{-}\left(\operatorname{Gauss}_{q, *}^{-}\right), \\
& \pi_{!}^{-}\left(\operatorname{Gauss}_{q, ! *}^{-}\right) \rightarrow \pi_{*}^{-}\left(\operatorname{Gauss}_{q, ! *}^{-}\right)
\end{aligned}
$$

and

$$
\pi_{!}^{-}\left(\operatorname{Gauss}_{q, !}^{-}\right) \rightarrow \pi_{*}^{-}\left(\operatorname{Gauss}_{q, !}^{-}\right)
$$

are isomorphisms.

This shows that the objects (0.11) are all perverse and we have

$$
\mathbb{D}_{\text {Conf }}^{\text {Verdier }}\left(\Omega_{q, \text { Whit }}^{\text {DK }}\right) \simeq \Omega_{q^{-1}, \text { Whit }}^{\text {Lus }} \text { and } \mathbb{D}_{\text {Conf }}^{\text {Verdier }}\left(\Omega_{q, \text { Whit }}^{\text {sml }}\right) \simeq \Omega_{q^{-1}, \text { Whit }}^{\text {sml }}
$$

\footnotetext{
${ }^{3}$ The connection to the construction of $\Omega_{q}^{\mathrm{Lus}}$ as $J_{\mathrm{Whit}}\left(\mathbf{1}_{\mathrm{Whit}}{ }_{\mathcal{G}_{q}^{G}}\left(\mathrm{Gr}_{G}\right)\right)$ can be seen from the material explained in (the optional) Sects. 5.5.5.7
} 
0.2.6. A rather simple calculation (see Proposition 6.2.2) shows that

$$
\left.\Omega_{q, \text { Whit }}^{\mathrm{DK}}\right|_{\text {Conf }} ^{\circ},\left.\Omega_{q, \text { Whit }}^{\text {sml }}\right|_{\text {Conf }} ^{\circ},\left.\Omega_{q, \text { Whit }}^{\text {Lus }}\right|_{\text {Conf }} ^{\circ}
$$

are all isomorphic to $\stackrel{\circ}{\Omega}_{q}$.

Our two main results, Theorems 6.2.5 and 6.2.6 assert that, under a certain restriction on $q$ (namely, $q$ should avoid small torsion, see Sect. 1.1.5 for what this means), these identifications extend (automatically, uniquely) to isomorphisms

$$
\Omega_{q, \mathrm{Whit}}^{\mathrm{DK}} \simeq \Omega_{q}^{\mathrm{DK}}, \Omega_{q, \mathrm{Whit}}^{\mathrm{Lus}} \simeq \Omega_{q}^{\mathrm{Lus}}
$$

and

$$
\Omega_{q, \mathrm{Whit}}^{\mathrm{sml}} \simeq \Omega_{q}^{\mathrm{sml}}
$$

respectively.

We note that the isomorphism (0.12) has been already established in Lys2 under somewhat more restrictive conditions on $q$.

0.2.7. We will outline the strategy of the proof of Theorems 6.2.5 and 6.2.6 in some detail in Sect. 0.3 below. Here we remark that, as we explain in Sects. 6.3 and Sect. 6.5 the statements of both these theorems are equivalent to one cohomological estimate.

Namely, let $\lambda \in \Lambda^{\text {neg }}-0$ be not one of the negative simple roots. Consider the intersection

$$
S^{0} \cap S^{-, \lambda} \subset \mathrm{Gr}_{G},
$$

where

$$
S^{0}=N((t)) \cdot 1 \text { and } S^{-, \lambda}=N^{-}((t)) \cdot t^{\lambda}
$$

are the positive and negative semi-infinite orbits on the affine Grassmannian.

The datum of $q$ defines a Kummer sheaf on $S^{0} \cap S^{-, \lambda}$, to be denoted $\Psi_{q, \lambda}$, see Sect. 6.3.6月.

Consider the compactly supported cohomology

$$
H_{c}^{i}\left(S^{0} \cap S^{-, \lambda}, \Psi_{q, \lambda} \stackrel{*}{\otimes} \chi^{*}(\exp )\right) .
$$

We have

$$
\operatorname{dim}\left(S^{0} \cap S^{-, \lambda}\right)=-\langle\lambda, \check{\rho}\rangle,
$$

and it is fairly easy to see that the top cohomology in (0.13), i.e., one for $i=-2\langle\lambda, \check{\rho}\rangle$, vanishes.

Now, the cohomology estimate mentioned above (one which is equivalent to Theorems 6.2.5 and 6.2.6) says that the cohomology (0.13) vanishes also in the sub-top degree

$$
i=-2\langle\lambda, \check{\rho}\rangle-1
$$

for $q$ that avoids small torsion.

This is our Theorem 6.3.8 (this is also Lys2, Theorem 1.1.5] for a more restrictive hypothesis on $q$ ).

Our proof of Theorems 6.2.5 6.2.6 and 6.3.8 makes use of quantum groups (this is while the proof in Lys2 is a direct geometric argument, but one that has to exclude more cases).

So in a certain sense, we have a somewhat crazy story: in order to prove the vanishing of the cohomology of a local system on a variety in the sub-top degree (say for $\ell$-adic sheaves over a ground field of positive characteristic), we use the structure theory of quantum groups (the quantum group in question is the quantization of the Langlands dual group $\check{G}$ ).

0.3. Strategy of proof: quantum groups creep in. We first explain how to prove Theorems 6.2.5 and 6.2.6 when the ground field $k$ has characteristic 0. In Sect. 0.3.4 we explain how we deduce from this the general case.

\footnotetext{
${ }^{4}$ In fact, if $q$ is of the form $\zeta \cdot q_{\mathbb{Z}}^{\min }$, where $q_{\mathbb{Z}}^{\min }$ is the minimal form on $\Lambda$ and $\zeta \in \mathfrak{Z}$, then $\Psi_{q, \lambda}$ is the pullback of the Kummer local system $\Psi_{\zeta}$ on $\mathbb{G}_{m}$ along a canonically defined invertible function $f_{\lambda}$ on $S^{0} \cap S^{-, \lambda}$, see Sect. 7.4.4
} 
0.3.1. The case when $q$ takes non-torsion values is easy to settle (in fact, in this case Theorem 6.3.8 is an easy calculation, which was performed already in Ga3]). So we will assume that $q$ takes values that are torsion in $\mathfrak{Z}$.

The proof of Theorems 6.2.5 and 6.2.6 proceeds by studying an additional piece of structure on the factorization algebras $\Omega_{q, \text { Whit }}^{\text {Lus }}$ (resp., $\Omega_{q}^{\text {Lus }}$ ), namely Lusztig's quantum Frobenius.

Let $\Lambda^{\sharp}$ be the "quantum Frobenius lattice" (see Sect. 4.1.1), and let Conf $f^{\sharp}$ be the corresponding configuration space. We can view Conf ${ }^{\sharp}$ as a semi-group acting on Conf. In this case we can talk about factorization algebras in $\operatorname{Perv}\left(\mathrm{Conf}^{\sharp}\right)$ acting on factorization algebras in $\mathrm{Shv}_{\mathcal{G}_{q}^{\text {Conf }}}(\mathrm{Conf})$, see Sect. 4.2.7

Following a recipe of $[\mathrm{BG} 2]$, we construct a factorization algebra $\Omega^{\mathrm{cl}, \sharp}$ in $\operatorname{Perv}\left(\mathrm{Conf}^{\sharp}\right)$.

By the quantum Frobenius structure on $\Omega_{q \text {,Whit }}^{\mathrm{Lus}}$ and $\Omega_{q}^{\mathrm{Lus}}$ we mean an action of $\Omega^{\mathrm{cl}, \sharp}$ on these algebras so that we have the isomorphisms

and

$$
\operatorname{Bar}\left(\Omega_{q, \text { Whit }}^{\text {Lus }}\right) \simeq \Omega_{q, \text { Whit }}^{\text {sml }}
$$

respectively.

Once we know that there exists quantum Frobenius structures on both $\Omega_{q \text {,Whit }}^{\text {Lus }}$ and $\Omega_{q}^{\text {Lus }}$, the proof of Theorem 6.2.6 is achieved by a Jordan-Holder series argument (see Sect. 6.6.4).

Now, the quantum Frobenius structure on $\Omega_{q, \text { Whit }}^{\text {Lus }}$ is constructed geometrically, using a metaplectic version of the construction from BG2, see Sect. 6.7

However, when it comes to $\Omega_{q}^{\text {Lus }}$, the way we define it does not a priori supply sufficient information to construct a quantum Frobenius structure on it. And it is here that quantum groups come in.

0.3.2. When considering $\Omega_{q}^{\text {Lus }}$, by Lefschetz principle, we may assume that the ground field is actually $\mathbb{C}$, and the sheaf theory in question is that of constructible sheaves in classical topology.

Let $\operatorname{Rep}_{q}(\check{T})$ be the category of representations of the quantum torus associated with $q$. I.e., this is a braided monoidal category, which as a monoidal category is isomorphic to $\operatorname{Rep}(\check{T})$, but the braiding is specified by $q$. Thus, it makes sense to talk about Hopf algebras in $\operatorname{Rep}_{q}(\check{T})$.

In Sect. 3.4 we recall a construction that attaches to a Hopf algebra $H$ in $\operatorname{Rep}_{q}(\check{T}$ ) (whose augmentation ideal is supported on coweights $\lambda \in \Lambda^{\text {neg }}-0$ with each coweight component finite-dimensional) a factorization algebra $\Omega_{H}$ in $\operatorname{Shv}_{\mathcal{G}_{q}^{\text {Conf }}}(\mathrm{Conf})$.

Applying this construction to Lusztig's, DeConcini-Kac and small versions of the (positive part of the) quantum group, we obtain factorization algebras

$$
\Omega_{q, \text { Quant }}^{\text {Lus }}, \Omega_{q, \text { Quant }}^{\text {DK }}, \Omega_{q, \text { Quant }}^{\text {sml }},
$$

respectively.

It follows by definition that we have a canonical identification

$$
\left.\Omega_{q, \text { Quant }}^{?}\right|_{\mathrm{Conf}} ^{\circ} \simeq \stackrel{\circ}{\Omega}_{q}
$$

for all three versions.

The t-exactness property of the construction $H \rightsquigarrow \Omega_{H}$ shows that the identification (0.17) extends (automatically, uniquely) to an isomorphism

$$
\Omega_{q, \text { Quant }}^{\mathrm{sml}} \simeq \Omega_{q}^{\mathrm{sml}}
$$

Further, in Theorem 3.6 .2 we show that the identification (0.17) extends to the entire Conf:

$$
\Omega_{q, \text { Quant }}^{\mathrm{DK}} \simeq \Omega_{q}^{\mathrm{DK}}
$$


(this is not difficult to prove). Finally, by Verdier duality, from (0.18) we obtain:

$$
\Omega_{q, \text { Quant }}^{\text {Lus }} \simeq \Omega_{q}^{\text {Lus }} .
$$

Thus, we have identified the pair of factorization algebras $\left(\Omega_{q}^{\mathrm{Lus}}, \Omega_{q}^{\mathrm{sml}}\right)$ with $\left(\Omega_{q, \mathrm{Quant}}^{\mathrm{Lus}}, \Omega_{q, \mathrm{Quant}}^{\mathrm{sml}}\right)$. Hence, in order to construct the quantum Frobenius structure on $\Omega_{q}^{\mathrm{Lus}}$, it is enough to do so for $\Omega_{q, \mathrm{Quant}}^{\mathrm{Lus}}$. The latter is given by Lusztig's Frobenius for quantum group 5 .

Remark 0.3.3. We should remark that the factorization algebras (0.16) are not new objects in mathematics. For example, $\Omega_{q, \text { Quant }}^{\mathrm{sml}}$ was the key object of study of the book BFS] (building on earlier works of V. Schechtman and A. Varchenko). In fact the contents of Sect. 3.4 summarize the relationship between $\Omega_{q, \text { Quant }}^{\text {sml }}$ and $u_{q}(\check{N})$ from loc.cit.

0.3.4. Let us now explain how to deduce Theorems 6.2.5 and 6.2.6 over an arbitrary ground field from the case of the ground field of characteristic 0 .

As we explained above, Theorems 6.2.5 and 6.2.6 are equivalent to Theorem 6.3.8 which asserts that the sub-top cohomology in (0.13) vanishes.

With no restriction of generality we can assume that $G$ is simple, and hence $q$ is of the form $\zeta \cdot q_{\mathbb{Z}}^{\min }$, where $q_{\mathbb{Z}}^{\min }$ is the minimal integer-valued quadratic form on $\Lambda$ and $\zeta$ is a torsion element in $\mathfrak{Z}$.

In Sect. 7 we prove Theorem 7.1 .2 which says that the validity of Theorem 6.3 .8 for a given $\operatorname{ord}(\zeta)$ is independent of the ground field.

To do so, we analyze irreducible components of $Z$ of $S^{0} \cap S^{-, \lambda}$ for which

$$
H_{c}^{i}\left(Z,, \Psi_{q, \lambda} \stackrel{*}{\otimes} \chi^{*}(\exp )\right)
$$

may potentially be non-zero for $i=-2\langle\lambda, \check{\rho}\rangle-1$.

We first single out components that we declare to be "under scrutiny", then among those some that we call "suspicious" (these two conditions do not depend on $\operatorname{ord}(\zeta)$ ), and finally those that we declare as "indicted". We then proceed to conviction and show that the presence of an indicted component indeed brings about the failure of Theorem 6.3.8 (for a given ord $(\zeta)$ ).

Thus, Theorem 7.1 .2 amounts to saying that for a given ord $(\zeta)$, the presence of indicted irreducible components is independent of the ground field. We prove this by showing that the property of being indicted can be expressed purely in terms of the structure of Kashiwara's crystal on the set

$$
\mathrm{B}(\lambda):=\cup_{\lambda^{\prime}} \operatorname{Irred}\left(S^{\lambda^{\prime}} \cap S^{-, \lambda}\right) .
$$

We conclude the proof by noticing that the crystal $B(\lambda)$ is independent of the characteristic of the ground field, due to Kashiwara's uniqueness theorem.

Remark 0.3.5. Let us emphasize that the idea to use the structure of Kashiwara's crystal on $B(\lambda)$ for the proof of Theorem 6.3 .8 originates in Lys2.

In fact, we follow the argument of loc.cit., but go one step further: in Lys2 one looks at the irreducible components that we call suspicious and rules them out for the specified values of ord $(\zeta)$. We go from "suspicious" and "indicted" and that puts a stricter bound on ord $(\zeta)$.

0.4. Organization of the paper. The actual order of exposition in the paper is somewhat different from how we have presented the contents in Sect. 0.2 We now briefly outline how the paper is organized section-by-section.

0.4.1. In Sect. 1 we introduce the setting of factorization algebras in (gerbe-twisted) sheaves on the configuration space and define the factorization algebras $\Omega_{q}^{\mathrm{Lus}}, \Omega_{q}^{\mathrm{sml}}$ and $\Omega_{q}^{\mathrm{DK}}$.

0.4.2. In Sect. 2 we state a number of theorems pertaining to the inductive construction of $\Omega_{q}^{\mathrm{DK}}$ by extending it across the main diagonal. The factorization algebra $\Omega_{q}^{\text {Lus }}$ will enjoy a Verdier dual behavior.

${ }^{5}$ It is here that we use the assumption that $q$ avoids small torsion. 
0.4.3. In Sect. 3 we introduce the setting of Hopf algebras in the braided monoidal category $\operatorname{Rep}_{q}(\check{T})$, and the relationship between these Hopf algebras and factorization algebras on Conf. Starting from the positive part of the (various versions of the) quantum group, we construct the factorization algebras $\Omega_{q, \text { Quant }}^{\text {Lus }}, \Omega_{q, \text { Quant }}^{\text {sml }}$ and $\Omega_{q, \text { Quant }}^{\text {DK }}$.

We establish the isomorphisms $\Omega_{q, \text { Quant }}^{\text {? }} \simeq \Omega_{q}^{?}$. We then use properties of quantum groups to prove the theorems announced in Sect. 2.

0.4.4. In Sect. 4 we introduce the quantum Frobenius.

We introduce the quantum Frobenius lattice $\Lambda^{\sharp}$ (along with the corresponding configuration space) $\mathrm{Conf}^{\sharp}$, and define what it means for a factorization algebra in $\operatorname{Shv}\left(\mathrm{Conf}{ }^{\sharp}\right)$ to act on a factorization algebra in $\operatorname{Shv}_{\mathcal{G}_{q} \text { Conf }}(\mathrm{Conf})$.

We then use the quantum Frobenius for $U_{q}^{\text {Lus }}(\check{N})$ to construct an action of a certain canonically defined factorization algebra $\Omega^{\mathrm{cl}, \sharp}$ in $\operatorname{Shv}\left(\operatorname{Conf}^{\sharp}\right)$ on $\Omega_{q, \text { Quant }}^{\mathrm{Lus}}$. From here we deduce the existence of a (canonically defined) action of $\operatorname{Shv}\left(\mathrm{Conf}^{\sharp}\right)$ on $\Omega_{q}^{\mathrm{Lus}}$.

0.4.5. In Sect. 5] we recall the definition of the (several versions of the) Zastava space. We introduce the (twisted) perverse sheaves

on $z^{-}$.

$$
\nabla_{q, Z}^{-}, \Delta_{q, Z}^{-}, \mathrm{IC}_{q, Z}^{-}
$$

After tensoring by the pullback of the exponential/Artin-Schreier character sheaf, we obtain the perverse sheaves

$$
\operatorname{Gauss}_{q, *}^{-}, \operatorname{Gauss}_{q, !}^{-}, \operatorname{Gauss}_{q, ! *}^{-}
$$

on $z^{-}$, see Remark 6.2 .3 for the explanation of the name "Gauss".

One of the key technical results here is Theorem 5.4.7 which says that the objects (0.19) extend cleanly along the open embedding

$$
z^{-} \hookrightarrow \bar{z}
$$

where $\bar{z}$ is the compactified Zastava space.

Finally, in Sects. 5.5 5.7 we present a more conceptual point of view on the construction of the objects (0.19). Namely, we show how they arise from factorization algebras in Whittaker and semiinfinite categories on $\mathrm{Gr}_{G}$.

0.4.6. In Sect. 6] we finally formulate and prove the main results of this paper.

We define the factorization algebras

$$
\Omega_{q, \text { Whit }}^{\mathrm{DK}}, \Omega_{q, \mathrm{Whit}}^{\mathrm{Lus}}, \Omega_{q, \mathrm{Whit}}^{\mathrm{sml}}
$$

by taking the direct image(s) of the objects (0.19) along the projection

$$
\pi^{-}: z^{-} \rightarrow \text { Conf. }
$$

We show that their restrictions to Conf identify with $\stackrel{\circ}{\Omega}_{q}$, and we state our main Theorems 6.2 .5 and 6.2.6. which say that the above identification over Conf extends to isomorphisms

$$
\Omega_{q, \text { Whit }}^{?} \simeq \Omega_{q}^{?}
$$

for $?=\mathrm{DK}$, Lus, sml.

We then show that Theorems 6.2.5 and 6.2.6 are both equivalent to Theorem 6.3.8, which states the sub-top cohomology in 0.13 vanishes.

Finally, we prove Theorems 6.2.5 and 6.2.6 (over a ground field of characteristic zero) using the quantum Frobenius and a certain trick involving Jordan-Holder series. 
0.4.7. Finally, in Sect. 7 we deduce the validity of Theorem 6.3.8 (and hence also that of Theorems 6.2.5 and 6.2.6) over an arbitrary ground field from the case when the ground field has characteristic 0.

The game here is to express the validity of Theorem 6.3.8 in terms of the structure of Kashiwara's crystal on the set of irreducible components of intersections of semi-infinite orbits.

0.4.8. In Sect. A we explain how to start with a sheaf theory $Y \mapsto \operatorname{Shv}(Y)$ with a field of coefficients e, and obtain from it a sheaf theory $Y \mapsto \operatorname{Shv}_{\llbracket \hbar \rrbracket}(Y)$ over e $\llbracket \hbar \rrbracket$.

This story is parallel (but simpler) to how one gets $\mathbb{Z}_{\ell}$-adic sheaves from $\mathbb{Z} / \ell^{n} \mathbb{Z}$-sheaves, which was systematically developed in GaLu, Sect. 2.3].

0.5. Notations and conventions. By and large, this paper follows the notational conventions from GLys1, GLys2.

0.5.1. In this paper our algebraic geometry takes place over an algebraically closed field, denoted $k$.

We will only need the classical (i.e., non-derived) algebraic geometry. We let $\operatorname{Sch}_{\mathrm{ft}}^{\text {aff }}$ denote the

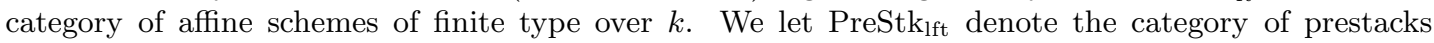
locally of finite type over $k$, i.e., the category of all functors

$$
\left(\mathrm{Sch}_{\mathrm{ft}}^{\mathrm{aff}}\right)^{\mathrm{op}} \rightarrow \infty \text {-Groupoids . }
$$

This category contains all schemes, ind-schemes, algebraic stacks, etc. (all locally of finite type).

That said, except for Sect. 0.5 .5 below, for the purposes of this paper we will only need groupoids with values in discrete $\infty$-Groupoids, i.e., sets, and the most general prestacks that we will consider are ind-schemes.

We let $X$ be a smooth connected curve over $k$.

0.5.2. We let $G$ be a semi-simple simply-connected groun 6 over $k$.

We let $\Lambda$ denote its coweight (=coroot lattice). Let $\check{\Lambda}$ denote the dual lattice (i.e., the weight lattice of $G$ ). Let $\check{\rho} \in \check{\Lambda}$ denote the half-sum of the positive roots.

Let $I$ denote the Dynkin diagram of $G$. For $i \in I$ we let $\alpha_{i} \in \Lambda$ denote the corresponding simple coroot. We let $\Lambda^{\text {pos }}$ (resp., $\Lambda^{\text {neg }}$ ) spanned by the elements $\alpha_{i}$ (resp., $-\alpha_{i}$ ).

For an element $\lambda \in \Lambda^{\text {pos }}$ (resp., $\Lambda^{\text {neg }}$ ) we will denote by $|\lambda|$ its length, i.e., $\langle\lambda, \check{\rho}\rangle$ (resp., $-\langle\lambda, \check{\rho}\rangle$ ).

0.5.3. We will work with DG categories over a field of coefficients e, assumed of characteristic 0. When we say "category" we will implicitly mean "DG category".

In this paper, unless specified otherwise, we will work with small (non ind-complete) DG categories.

Given a DG category $\mathbf{C}$, we can talk about t-structures on it. Given a t-structure, we will denote by $\mathbf{C}^{\odot}$ its heart.

\footnotetext{
${ }^{6}$ Although the FLE can be formulated for an arbitrary reductive $G$, the current paper is dealing with the vacuum objects, namely, the corresponding factorization algebras, which only depend on the root system of $G$. Hence for the purposes of this paper, it is enough to consider the case of $G$ simply-connected. This assumption has the additional advantage of streamlining to discussion of the geometric metaplectic data/factorization gerbes on the configuration spaces: for $G$ simply-connected these gerbes are uniquely recovered from the datum of a quadratic form $q$.
} 
0.5.4. Sheaf theories. We will work with one of the sheaf theories

$$
Y \mapsto \operatorname{Shv}(Y), \quad\left(\operatorname{Sch}_{\mathrm{ft}}^{\mathrm{aff}}\right)^{\mathrm{op}} \rightarrow \text { DGCate }_{\mathrm{e}}
$$

(see GLys2, Sect. 0.8.8]) from the following list:

- When $k=\mathbb{C}$ we can take $\operatorname{Shv}(-)$ to be constructible sheaves in the classical topology with coefficients in a field e of characteristic 0;

- Over an arbitrary ground field, we can take $\operatorname{Shv}(-)$ to be constructible $\overline{\mathbb{Q}}_{\ell}$-adic sheaves (in this case the field e of coefficients is $\overline{\mathbb{Q}}_{\ell}$ );

- Over a ground field $k$ of characteristic 0 , we can take $\operatorname{Shv}(-)$ to be the category of holonomic D-modules (in this case the field e of coefficients is $k$ ).

We extend the assignment (0.20) to a functor

$$
\left(\text { PreStk }_{\text {lft }}^{\text {aff }}\right)^{\text {op }} \rightarrow \text { DGCat }_{e}
$$

by the procedure of GLys2, Sect. 0.8.9].

0.5.5. Let 1 -LS be the commutative group object in PreStk $\mathrm{lft}_{\mathrm{ft}}$ that assigns to $Y \in \operatorname{Sch}_{\mathrm{ft}}^{\text {aff }}$ the category of 1-dimensional local systems on $Y$.

Let Ge be the Zariski sheafification $B_{\mathrm{Zar}}(1-\mathrm{LS})$ of $B(1-\mathrm{LS})$. For a prestack $y$, by $\mathrm{e}^{\times}$-gerbe on $y$ we will mean a map $y \rightarrow$ Ge.

Given a $e^{\times}$-gerbe $\mathcal{G}$ on $y$, we can form a twisted category $\operatorname{Shv}_{\mathcal{G}}(y)$, see [GLys1, Sect. 1.7].

If $Y$ is a scheme, the category $\operatorname{Shv}_{\mathcal{G}}(y)$ is equipped with a t-structure and we can consider the abelian category $\operatorname{Perv}_{\mathcal{G}}(y)$.

0.5.6. In each of the above three examples of sheaf theories, we let $\mathfrak{Z}$ be the following abelian group:

- For sheaves in the classical topology, we let $\mathfrak{Z}:=\mathrm{e}^{\times}$;

- For $\overline{\mathbb{Q}}_{\ell}$-adic sheaves, we let $\mathfrak{Z}:=\overline{\mathbb{Z}}_{\ell}^{\times}$;

- For D-modules, we let $\mathfrak{Z}:=k / \mathbb{Z}$.

In each of the above cases, an element $\zeta \in \mathfrak{Z}$ defines a Kummer character sheaf on $\mathbb{G}_{m}$, to be denoted $\Psi_{\zeta}$.

0.5.7. Furthermore, let $\mathcal{L}$ be a line bundle on $y$ and let $\zeta$ be an element in $\mathfrak{Z}$. From this pair we obtain a canonically defined $e^{\times}$-gerbe, to be denoted $\mathcal{L}^{\zeta}$.

Indeed, by definition, it is sufficient to perform this construction for $y=Y \in \mathrm{Sch}_{\mathrm{ft}}^{\text {aff }}$. In this case, the sought-for map

$$
Y \rightarrow B_{\mathrm{Zar}}(1-\mathrm{LS})
$$

is such that for every $U \subset Y$ and a trivialization of $\left.\mathcal{L}\right|_{U}$, the corresponding map

$$
U \rightarrow Y \rightarrow B \text { Zar }(1-\mathrm{LS})
$$

acquires a trivialization, and a change of the trivialization of $\left.\mathcal{L}\right|_{U}$ by $f_{U}: U \rightarrow \mathbb{G}_{m}$ results in the change of the trivialization of (0.21) by

$$
U \stackrel{f_{U}}{\rightarrow} \mathbb{G}_{m} \stackrel{\Psi_{\zeta}}{\rightarrow} 1-\mathrm{LS}
$$

0.6. Acknowledgements. The author would like to thank P. Etingof, I. Heckenberger, D. Nakano and I. Angiono for their help with quantum groups at small roots of unity.

The author is grateful to S. Lysenko for his collaboration on the FLE and for sharing ideas related to this work.

The author's thinking about the subject of this paper has its origins in the book [BFS]; he is grateful to its authors for inspiration and discussions we have had over many years.

A major part of this work was written while the author was holding the I. M. Gelfand chair at IHES.

The author's research is supported by NSF grant DMS-1707662. 


\section{Factorization algebras atTached to QuAdratic Forms}

In this section we will introduce a quantum parameter $q$, recall the construction of the factorization gerbe $\mathcal{G}_{q}^{\text {Conf }}$ on the configuration space Conf, and define the factorization algebras

$$
\Omega_{q}^{\mathrm{sml}}, \Omega_{q}^{\mathrm{DK}}, \Omega_{q}^{\mathrm{Lus}},
$$

which are $\mathcal{G}_{q}^{\mathrm{Conf}}$-twisted perverse sheaves on Conf, equipped with a factorization structure.

\subsection{The parameters.}

1.1.1. We start with a datum of a quadratic form $q$ on $\Lambda$ with values in $\mathfrak{Z}$ (see Sect. 0.5.6 for what the symbol $\mathfrak{Z}$ stands for), which is $W$-invariant and restricted. The latter means the following:

Let

$$
b: \Lambda \otimes \Lambda \rightarrow \mathfrak{Z}
$$

be the associated symmetric bilinear form, i.e.,

$$
b(\lambda, \mu)=q(\lambda+\mu)-q(\lambda)-q(\mu) .
$$

We say that $q$ is restricted if $b$ satisfies the following identity

$$
b(\alpha, \lambda)=\langle\lambda, \check{\alpha}\rangle \cdot q(\alpha)
$$

for every coroot $\alpha$ and $\lambda \in \Lambda$.

Remark 1.1.2. It is easy to see that the identity

$$
2 b(\alpha, \lambda)=2\langle\lambda, \check{\alpha}\rangle \cdot q(\alpha)
$$

is a formal consequence of $W$-invariance. But formula (1.1) is not; it is easy to produce a counterexample for the group $S L_{2} \times \mathbb{G}_{m}$.

1.1.3. It is shown in GLys1, Sect. 3.2.4] that any restricted $q$ can be written as

$$
\Lambda \stackrel{q \mathbb{Z}}{\rightarrow} \mathbb{Z} \stackrel{\zeta}{\rightarrow} \mathfrak{Z}
$$

for some element $\zeta \in \mathfrak{Z}$ and an integer-valued $W$-invariant quadratic form $q_{\mathbb{Z}}$ on $\Lambda$. Note that it follows from Remark 1.1.2 that $q_{\mathbb{Z}}$ automatically satisfies (1.1).

In particular, for every simple factor of our root system we have

$$
q\left(\alpha_{l}\right)=d \cdot q\left(\alpha_{s}\right)
$$

where $\alpha_{l}$ (resp., $\alpha_{s}$ ) is any long (resp., short) coroot, and $d$ is the lacing number (the ratio of the squares of the lengths).

1.1.4. We shall say that $q$ is torsion-valued if the values of $q$ in $\mathfrak{Z}$ are torsion.

We shall say that $q$ is non-torsion valued if for all coroots $\alpha$, the elements $q(\alpha)$ are non-torsion. By (1.2), this condition is enough to check for one coroot in each simple factor.

Note that when our sheaf theory is that of sheaves in the classical topology and so $\mathfrak{Z}$ is the multiplicative group of the field of coefficients which we denote e, "torsion" means being a root of unity in $\mathrm{e}^{\times}$. So in the usual terminology of quantum groups, "torsion vs. non-torsion" means "roots of unity vs. non-roots of unity".

We shall say that $q$ is non-degenerate if $q(\alpha) \neq 0$ for all coroots $\alpha$. By (1.2), this condition is enough to check for the long coroot in each simple factor of the root system.

The condition of non-degeneracy means that we are avoiding what is called "the quasi-classical case" in [Lus, Sect. 33.2] along any of the roots. 
1.1.5. We shall say that $q$ avoids small torsion if for every simple factor in our root system and (any) long coroot $\alpha_{l}$ in it we have

$$
\operatorname{ord}\left(q\left(\alpha_{l}\right)\right) \geq d+1
$$

where $d=1,2,3$ is the lacing number of that simple factor. This is equivalent to Lus, Condition $35.1 .2(\mathrm{a})]$.

Note that if $d=1$, the above assumption is equivalent to $q$ being non-degenerate. For $d=2$, the assumption is equivalent to $\operatorname{ord}\left(\alpha_{l}\right) \neq 2$. For $d=3$, the assumption is equivalent to $\operatorname{ord}\left(\alpha_{l}\right) \neq 2,3$.

The key results of this paper (Theorems 6.2.5 and 6.2.6) will use this assumption. On the quantum group side, this assumption ensures a relationship between $U_{q}^{\text {Lus }}(\check{N})$ and $u_{q}(\check{N})$ via the quantum Frobenius, i.e., that (4.30) is a short exact sequence of Hopf algebras.

Remark 1.1.6. From a certain point of view, when $q$ does not avoid small torsion, the factorization algebras we will construct, namely, $\Omega_{q}^{\mathrm{Lus}}, \Omega_{q}^{\mathrm{DK}}, \Omega_{q}^{\mathrm{sml}}$ are not quite the right objects to consider.

The more relevant ones are the objects $\Omega_{q, \mathrm{Whit}}^{\mathrm{Lus}}, \Omega_{q, \mathrm{Whit}}^{\mathrm{DK}}, \Omega_{q, \mathrm{Whit}}^{\mathrm{sml}}$, constructed in Sect. 6 . And these objects are indeed different (for example, for $G=G_{2}$ and $\operatorname{ord}\left(q\left(\alpha_{s}\right)\right)=\operatorname{ord}\left(q\left(\alpha_{l}\right)\right)=2$, see Remark 7.5.6).

From this point of view, the quantum algebras $U_{q}^{\mathrm{Lus}}(\check{N}), U_{q}^{\mathrm{DK}}(\check{N}), \mathfrak{u}_{q}(\check{N})$ are not quite the right objects either (at these very small roots of unity).

1.1.7. Note that the pairing

$$
b: \Lambda \otimes \Lambda \rightarrow \mathfrak{Z}
$$

canonically extends to a pairing

$$
b: \Lambda \otimes \Lambda_{\mathrm{ad}} \rightarrow \mathfrak{Z}
$$

where $\Lambda_{\mathrm{ad}}$ is the coroot lattice of the adjoint quotient of $G$.

Namely, we set

$$
b\left(\alpha_{i}, \mu\right):=\left\langle\mu, \check{\alpha}_{i}\right\rangle \cdot q\left(\alpha_{i}\right), \quad i \in I .
$$

Alternatively, let $n$ be an integer so that $n \cdot \Lambda_{\text {ad }} \subset \Lambda$. Let us write $q$ as $q_{\mathbb{Z}} \cdot \zeta$, and choose an $n$-th root $\zeta^{\frac{1}{n}}$ of $\zeta$. Then we have

$$
b(\lambda, \mu)=b_{\mathbb{Z}}(\lambda, n \cdot \mu) \cdot \zeta^{\frac{1}{n}} .
$$

By (1.1), this gives the same value as (1.3) on the simple coroots. Furthermore, this implies that the formula

$$
b(\alpha, \lambda):=\langle\lambda, \check{\alpha}\rangle \cdot q(\alpha)
$$

holds for all coroots.

1.1.8. In view of the above, we have a well-defined homomorphism

$$
\lambda \in \Lambda \mapsto b(\lambda, \rho) \in \mathfrak{Z},
$$

where $\rho$ is half-sum of positive coroots. Namely,

$$
b\left(\alpha_{i}, \rho\right):=q\left(\alpha_{i}\right)
$$

The following is simple combinatorial statement:

Lemma 1.1.9. For $\lambda \in \Lambda^{\text {neg }}$, consider the element

$$
q(\lambda)+b(\lambda, \rho) \in \mathfrak{Z} .
$$

The element (1.5) vanishes for all $\lambda$ of the form $w(\rho)-\rho, w \in W$.

\subsection{The configuration space.}

\footnotetext{
${ }^{7}$ For the explanation why these objects are better behaved, see Remark 6.6.3
} 
1.2.1. Let Conf denote the configuration space of points of $x$ weighted by elements of $\Lambda^{\text {neg }}-0$, i.e., expressions of the form

$$
\Sigma \lambda_{n} \cdot x_{n}, \quad \lambda_{n} \in \Lambda^{\text {neg }}-0, \quad x_{n^{\prime}} \neq x_{n^{\prime \prime}}
$$

We have

$$
\text { Conf }=\underset{\lambda \in \Lambda^{\text {neg }}-0}{\sqcup} \operatorname{Conf}^{\lambda}
$$

according to the total degree.

Each connected component $\operatorname{Conf}^{\lambda}$, also denoted $X^{\lambda}$, is a partially symmetrized power of $X$. Namely, for

$$
\lambda=\sum_{i \in I} \cdot\left(-n_{i}\right) \cdot \alpha_{i}
$$

(where we recall that $I$ is the set of vertices of the Dynkin diagram), we have

$$
\operatorname{Conf}^{\lambda}:=X^{\lambda} \simeq \prod_{i \in I} X^{\left(n_{i}\right)} .
$$

1.2.2. We let

$$
\text { Conf } \stackrel{j}{\hookrightarrow} \text { Conf }
$$

denote the open locus corresponding to the condition that in (1.6) all $\lambda_{n}$ are negative simple roots.

In other words, each connected component $\operatorname{Conf}^{\lambda}$ is the complement of the diagonal divisor in (1.8).

1.2.3. In what follows we will denote by

$$
(\text { Conf } \times \text { Conf })_{\text {disj }} \subset \text { Conf } \times \text { Conf }
$$

the disjoint locus, i.e., the open subset consisting of those pairs

$$
\left(\Sigma \lambda_{n} \cdot x_{n}, \Sigma \lambda_{n^{\prime}}^{\prime} \cdot x_{n^{\prime}}^{\prime}\right),
$$

for which all $x_{n}$ and $x_{n^{\prime}}^{\prime}$ are pairwise distinct.

Similarly, we will use the notation

$$
\left(\text { Conf }^{\times n}\right)_{\operatorname{disj}} \subset \operatorname{Conf}^{\times n}
$$

for the $n$-fold product.

1.2.4. We let

$$
\text { add : Conf } \times \text { Conf } \rightarrow \text { Conf }
$$

denote the addition map, and let $\operatorname{add}_{n}$ be its $n$-fold version.

Note that add $\left(\right.$ resp., $\left.\operatorname{add}_{n}\right)$ is étale when restricted to $(\text { Conf } \times \text { Conf })_{\text {disj }}\left(\operatorname{resp} .,\left(\text { Conf }^{\times n}\right)_{\text {disj }}\right)$.

1.2.5. For an element $\lambda \in \Lambda^{\text {neg }}-0$, let $\Delta_{\lambda}$ denote the corresponding main diagonal

$$
X \rightarrow \operatorname{Conf}^{\lambda} \subset \text { Conf }, \quad x \mapsto \lambda \cdot x .
$$

For a fixed point $x \in X$, we will use the notation $\iota_{\lambda}$ for the corresponding point $\lambda \cdot x \in$ Conf $^{\lambda} \subset$ Conf.

\subsection{Gerbes on the configuration space.}

1.3.1. According to GLys1, Sect. 4.5.2], to $q$ one canonically attaches a geometric metaplectic data $\mathcal{G}_{q}^{T}$ for the torus $T$. It is uniquely characterized by the following two requirements:

-The associated quadratic form (see GLys1, Sects. 4.2.1 and 4.2.8]) equals $q$;

-For $\lambda \in \Lambda$, the gerbe $\mathcal{G}_{q}^{\lambda}$ on $X$ (see GLys1, Sect. 4.2.1]) is trivialized for $\lambda$ being each of the negative simple roots.

In what follows, for a fixed point $x \in X$ we let $\mathcal{G}_{q, x}^{\lambda}$ denote the fiber of $\mathcal{G}_{q}^{\lambda}$ at $x$; this is a plain $\mathrm{e}^{\times}$-gerbe. 
1.3.2. By GLys2, Sect. 4.6.5], to $\mathcal{G}_{q}^{T}$ we attach a factorization gerbe, denoted $\mathcal{G}_{q}^{\text {Conf }}$ on Conf.

We recall that the datum of factorization amounts to an isomorphism

$$
\left.\left.\mathcal{G}_{q}^{\text {Conf }} \otimes \mathcal{G}_{q}^{\text {Conf }}\right|_{(\operatorname{Conf} \times \text { Conf })_{\text {disj }}} \simeq \operatorname{add}^{*}\left(\mathcal{G}_{q}^{\text {Conf }}\right)\right|_{(\operatorname{Conf} \times \text { Conf })_{\text {disj }}} .
$$

The isomorphism (1.10) is endowed with a datum of commutativity and associativity for iterated isomorphisms

$$
\left.\left.\left.\left(\mathcal{G}_{q}^{\text {Conf }}\right)^{\bigotimes n}\right|_{(\operatorname{Conf} \times n}\right)\left._{\mathrm{disj}} \simeq \operatorname{add}_{n}^{*}\left(\mathcal{G}_{q}^{\text {Conf }}\right)\right|_{(\operatorname{Conf} \times n}\right)_{\text {disj }}, \quad n \in \mathbb{N} .
$$

Let us spell out the construction $\mathcal{G}_{q}^{T} \rightsquigarrow \mathcal{G}_{q}^{\text {Conf }}$ explicitly, in terms of [GLys1, Sect. 4.2].

1.3.3. According to loc. cit., the data of $\mathcal{G}_{q}^{T}$ attaches to a finite set $J$ and a map $\lambda^{J}: J \rightarrow \Lambda$, a gerbe $\mathcal{G}_{q}^{\lambda^{J}}$ on $X^{J}$.

For $J=\{*\}$ and $\lambda^{J}$ corresponding to $\lambda \in \Lambda$, we recover the gerbe $\mathcal{G}_{q}^{\lambda}$ on $X$, mentioned above.

For $J=\{1,2\}$ and $\lambda^{J}$ given by a pair of elements $\lambda_{1}, \lambda_{2} \in \Lambda$, the factorization structure on $\mathcal{G}_{q}^{T}$ defines an isomorphism

$$
\left.\mathcal{G}_{q}^{\lambda_{1}, \lambda_{2}}\right|_{X \times X-\Delta}=\left.\mathcal{G}_{q}^{\lambda_{1}} \otimes \mathcal{G}_{q}^{\lambda_{2}}\right|_{X \times X-\Delta}
$$

which extends to an isomorphism

$$
\mathcal{G}_{q}^{\lambda_{1}, \lambda_{2}} \simeq\left(\mathcal{G}_{q}^{\lambda_{1}} \otimes \mathcal{G}_{q}^{\lambda_{2}}\right) \otimes \mathcal{O}(-\Delta)^{b\left(\lambda_{1}, \lambda_{2}\right)},
$$

where:

- for a line bundle $\mathcal{L}$ on a scheme $y$ and an element $\zeta \in \mathfrak{Z}$, we denote by $\mathcal{L}^{\zeta}$ the corresponding $\mathrm{e}^{\times}$-gerbe on $y$, see Sect. 0.5 .7

- $b$ is the symmetric bilinear form attached to $q$ (in fact, (1.11) shows how to recover $b$ starting from $\left.\mathcal{G}_{q}^{T}\right)$.

The assignment $\left(J, \lambda^{J}\right) \rightsquigarrow \mathcal{G}^{\lambda^{J}}$ is functorial in $\left(J, \lambda^{J}\right)$. In particular, $\mathcal{G}_{q}^{\lambda^{J}}$ is equivariant with respect to the group $\operatorname{Aut}\left(J, \Lambda^{J}\right)$.

The above structure of equivariance has the following property. Let again $J=\{1,2\}$, and $\lambda^{J}$ be the constant map with value $\lambda$. Then the induced structure of equivariance on

$$
\left.\mathcal{G}_{q}^{\lambda, \lambda}\right|_{\Delta}
$$

with respect to $\mathbb{Z} / 2 \mathbb{Z}$ is equipped with a trivialization (the latter makes sense as $\mathbb{Z} / 2 \mathbb{Z}$ acts trivially on the underlying scheme $\Delta$ ).

It is explained in GLys1, Sect. 4.2.8], this datum of trivialization exhibits $b(\lambda, \lambda)$ as $2 \times$ some other element of $\mathfrak{Z}$; this other element is the value $q(\lambda)$ of $q$ on $\lambda$.

Equivalently, this datum of trivialization is equivalent to descending the gerbe $\mathcal{G}_{q}^{\lambda, \lambda}$ on $X^{2}$ to a gerbe on $X^{(2)}$, which identifies with

$$
\left(\mathcal{G}_{q}^{\lambda}\right)^{(2)} \otimes \mathcal{O}\left(-\Delta^{\prime}\right)^{q(\lambda)}
$$

where:

- $\mathcal{G}^{(n)}$ is the gerbe on $X^{(n)}$ given by the $n$th symmetric power ${ }^{8}$ of a given gerbe $\mathcal{G}$ on $X$;

- $\Delta^{\prime}$ is the diagonal divisor in $X^{(2)}$.

Let now $\left(J, \lambda^{J}\right)$ be arbitrary. Let $X^{(J)}$ be the partially symmetrized power of the curve equal to the (GIT) quotient of $X^{J}$ by $\operatorname{Aut}\left(J, \Lambda^{J}\right)$. From the above datum of trivialization of the equivariance structure on the diagonals, we obtain that the gerbe $\mathcal{G}_{q}^{\lambda^{J}}$ canonically descends to a gerbe $\mathcal{G}_{q}^{\left(\lambda^{J}\right)}$ on $X^{(J)}$.

\footnotetext{
${ }^{8}$ I.e, if we trivialize $\mathcal{G}$ Zariski-locally on $X$, the gerbe $\mathcal{G}^{(n)}$ also acquires a trivialization; a change of the trivialization of $\mathcal{G}$ by a 1-dimensional local system $E$ on $X$ results in the change of trivialization of $\mathcal{G}^{(n)}$ by $E^{(n)}$, where the latter is a well-defined 1-dimensional local system on $X^{(n)}$.
} 
1.3.4. We are now ready to describe $\mathcal{G}_{q}^{\text {Conf }}$ explicitly in terms of $\mathcal{G}_{q}^{T}$.

For $\lambda \in \Lambda^{\text {neg }}$ written as (1.7), set

$$
J=\underset{i \in I}{\sqcup}\left\{1, \ldots, n_{i}\right\}
$$

and let $\lambda^{J}$ be such that it sends each $\left\{1, \ldots, n_{i}\right\}$ to $-\alpha_{i}$. Note that we have a natural identification

$$
\operatorname{Conf}^{\lambda}=X^{(J)} \text {. }
$$

Then with respect to this identification, we have

$$
\left.\mathcal{G}_{q}^{\text {Conf }}\right|_{\operatorname{Conf}^{\lambda}}=\mathcal{G}_{q}^{\left(\lambda^{J}\right)}
$$

1.3.5. Here is an even more explicit description of $\mathcal{G}_{q}^{\text {Conf }}$ in terms of $q$.

Recall (see Sect. 1.3.1) that each $\mathcal{G}_{q}^{-\alpha_{i}}$ is equipped with a trivialization.

This trivialization uniquely extends to a trivialization of the restriction of $\mathcal{G}_{q}^{\text {Conf }}$ to

$$
\text { Conf } \subset \text { Conf }
$$

in a way compatible with factorization.

Using (1.11) and (1.12), we obtain that for $\lambda \in \Lambda^{\text {neg }}$ written as (1.7) (so that Conf $^{\lambda}$ is written as in (1.8)), we have:

$$
\left.\mathcal{G}_{q}^{\text {Conf }}\right|_{\text {Conf }^{\lambda}}=\left(\underset{i}{\otimes} \mathcal{O}\left(-\Delta_{i}^{\prime}\right)^{q\left(\alpha_{i}\right)}\right) \bigotimes\left(\underset{i \neq j}{\otimes} \mathcal{O}\left(-\Delta_{i, j}\right)^{b\left(\alpha_{i}, \alpha_{j}\right)}\right)
$$

where:

- $\Delta_{i}^{\prime}$ denotes the diagonal divisor in $X^{\left(n_{i}\right)}$;

- The product in the second factor goes over the set of unordered pairs of distinct simple coroots, and $\Delta_{i, j}$ denotes the incidence divisor in $X^{\left(n_{i}\right)} \times X^{\left(n_{i}\right)}$;

1.3.6. Restricting the RHS of formula (1.13) to the main diagonal, we obtain that the gerbe $\mathcal{G}_{q}^{\lambda}$ on $X$ identifies with

$$
\omega^{q(\lambda)+b(\lambda, \rho)}
$$

see Sect. 1.1.8 for the meaning of $b(\lambda, \rho)$.

In particular, from Lemma 1.1.9 we obtain that $\mathcal{G}_{q}^{\lambda}$ is canonically trivial for $\lambda$ of the form $w(\rho)-\rho$.

1.3.7. Let $f: X_{1} \rightarrow X_{2}$ be an étale morphism of curves, and consider the corresponding map of the configuration spaces

$$
f_{\text {Conf }}: \operatorname{Conf}_{1} \rightarrow \operatorname{Conf}_{2} \text {. }
$$

Let Conf $_{1, e t} \subset \operatorname{Conf}_{1}$ be the locus on which $f_{\text {Conf }}$ is étale. Note that this locus always contains the main diagonal as well as the complement to the diagonal divisor.

Let $\left(\mathcal{G}_{q}^{\text {Conf }}\right)_{1}$ (resp., $\left.\left(\mathcal{G}_{q}^{\text {Conf }}\right)_{2}\right)$ denote the corresponding gerbe on Conf 1 (resp., Conf 2 ). It follows from formula (1.13) that the tautological isomorphism

$$
\left.\left.\left(\mathcal{G}_{q}^{\text {Conf }}\right)_{1}\right|_{\mathrm{Conf}_{1}} ^{\circ} \simeq f_{\text {Conf }}^{*}\left(\left(\mathcal{G}_{q}^{\text {Conf }}\right)_{2}\right)\right|_{\operatorname{Conf}_{1}} ^{\circ}
$$

extends (automatically uniquely) to an isomorphism

$$
\left.\left.\left(\mathcal{G}_{q}^{\text {Conf }}\right)_{1}\right|_{\text {Conf }_{1, e t}} \simeq f_{\text {Conf }}^{*}\left(\left(\mathcal{G}_{q}^{\text {Conf }}\right)_{2}\right)\right|_{\mathrm{Conf}_{1, e t}} .
$$


1.3.8. Assume for a second that $X=\mathbb{A}^{1}$, with a chosen coordinate $t$. This choice of coordinate makes all the divisors $\Delta_{i}$ and $\Delta_{i, j}$ principal, i.e., it gives rise to a trivialization of the line bundles $\mathcal{O}\left(-\Delta_{i}\right)$ and $\mathcal{O}\left(-\Delta_{i, j}\right)$, appearing in formula (1.13). In particular, we obtain that a choice of coordinate defines a trivialization of the gerbe $\mathcal{G}_{q}^{\text {Conf }}$.

Note, however, that this trivialization is incompatible with the canonical trivialization of $\left.\mathcal{G}_{q}^{\text {Conf }}\right|_{\text {Conf }} ^{\circ}$. The discrepancy is given by a local system equal to the tensor product

$$
\left(\otimes_{i} f_{i}^{*}\left(\Psi_{q\left(\alpha_{i}\right)}\right)\right) \bigotimes\left(\underset{i, j}{\otimes} f_{i, j}^{*}\left(\Psi_{b\left(\alpha_{i}, \alpha_{j}\right)}\right)\right)
$$

where:

- For $\zeta \in \mathfrak{Z}$, we denote by $\Psi_{\zeta}$ the corresponding Kummer local system on $\mathbb{G}_{m}$ (see Sect. 0.5.6);

- $f_{i}$ (resp., $f_{i, j}$ ) is the (invertible) function on $X^{\lambda}$ given by the generator of the ideal of $\Delta_{i}$ (resp., $\left.\Delta_{i, j}\right)$.

\subsection{Factorization algebras.}

1.4.1. The object of study in this paper is factorization algebras in the category of gerbe-twisted perverse sheaves 9 on Conf (resp., Conf).

1.4.2. In the untwisted case, such a factorization algebra is an object $\mathcal{A} \in \operatorname{Perv}(\mathrm{Conf})$, equipped with an isomorphism

$$
\left.\left.\mathcal{A} \otimes \mathcal{A}\right|_{(\operatorname{Conf} \times \operatorname{Conf})_{\mathrm{disj}}} \simeq \operatorname{add}^{!}(\mathcal{A})\right|_{(\operatorname{Conf} \times \operatorname{Conf})_{\mathrm{disj}}},
$$

which is commutative and associative in a natural sense.

Note that in the above formula $\left.\operatorname{add}^{!}(\mathcal{A})\right|_{(\operatorname{Conf} \times \operatorname{Conf})_{\text {disj }}}$ is perverse, since add $\left.\right|_{(\operatorname{Conf} \times \text { Conf })_{\text {disj }}}$ is étale.

1.4.3. Let now $\mathcal{G}^{\text {Conf }}$ be a gerbe over Conf, equipped with a factorization structure (see Sect. 1.3.2 for what this means). Then, due to the factorization structure on $\mathcal{G}$ we can talk about objects of $\operatorname{Perv}_{\mathcal{G}}(\mathrm{Conf})$, equipped with a factorization structure.

Indeed, in this case the left-hand side of (1.17) is an object of

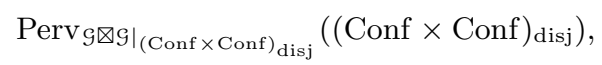

and the right-hand side is an object of

$$
\operatorname{Perv}_{\left.\operatorname{add}^{*}(\mathcal{G})\right|_{(\operatorname{Conf} \times \text { Conf })} \text { disj }}\left((\text { Conf } \times \text { Conf })_{\text {disj }}\right),
$$

but the two gerbes are identified due to (1.10).

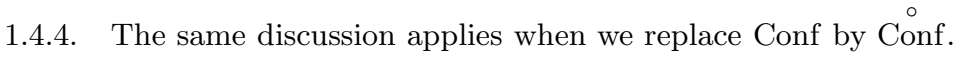

\subsection{The factorization algebra on the open part.}

1.5.1. Consider first the untwisted category $\operatorname{Perv}\left(\mathrm{Conf}^{\circ}\right)$. We let $\stackrel{\circ}{\Omega}$ be the factorization algebra in it, uniquely determined by the condition that

$$
\left.\stackrel{\circ}{\Omega}\right|_{\mathrm{Conf}^{-\alpha_{i}}} \simeq \mathrm{e}_{X}[1] .
$$

\footnotetext{
${ }^{9}$ See Sect. 0.5 .5 for what this means.
} 
1.5.2. Explicitly, recall that

$$
\text { Conf }=\underset{\lambda}{\sqcup} \operatorname{Conf}^{\lambda}=\underset{\lambda}{\sqcup}\left(\stackrel{\circ}{X^{\lambda}}-\Delta\right),
$$

where $\Delta$ is the diagonal divisor in each $\stackrel{\circ}{X}^{\lambda}$. I.e., for $\lambda$ written as (1.7), we have:

$$
\Delta=\left(\underset{i}{\cup} \Delta_{i}^{\prime}\right) \bigcup\left(\cup_{i, j} \Delta_{i, j}\right) .
$$

Then

identifies with the restriction of

$$
\left.\stackrel{\circ}{\Omega}\right|_{\operatorname{Conf}^{\lambda}}
$$

$$
\underset{i}{\bigotimes} \operatorname{sign}^{n_{i}}\left[n_{i}\right],
$$

where sign ${ }^{n}$ denotes the sign local system on $X^{(n)}-\Delta$ (the cohomological shift by $[n]$ makes $\operatorname{sign}^{n}[n]$ into a perverse sheaf).

1.5.3. Recall now that the restriction of the factorization gerbe $\mathcal{G}_{q}^{\text {Conf }}$ to $\stackrel{\circ}{\circ}$ onf admits a canonical trivialization. Hence, we can identify $\operatorname{Perv}_{\mathcal{S}_{q}^{\text {Conf }}}($ Conf $)$ with $\operatorname{Perv}($ Conf $)$.

Let $\stackrel{\circ}{\Omega}_{q} \in \operatorname{Perv}_{\mathcal{S}_{q}^{\text {Conf }}}($ Conf) be the image of $\stackrel{\circ}{\Omega}$ under the equivalence

$$
\operatorname{Perv}_{\mathcal{G}_{q}^{\text {Conf }}}(\text { Conf }) \simeq \operatorname{Perv}(\text { Conf }) .
$$

1.6. Construction of the "small" factorization algebra $\Omega_{q}^{\mathrm{sml}}$. When discussing the various versions of the quantum factorization algebra $\Omega_{q}$, we will assume that $q$ is non-degenerate (see Sect. 1.1.4 for what this means) 10 .

This assumption will be in effect for the duration of this subsection and the next one.

1.6.1. We define the factorization algebra $\Omega_{q}^{\mathrm{sml}} \in \operatorname{Perv}_{\mathcal{S}_{q}^{\text {Conf }}}($ Conf $)$

$$
\Omega_{q}^{\mathrm{sml}}:=j_{! *}\left(\stackrel{\circ}{\Omega}_{q}\right) .
$$

We emphasize that in the above formula, the Goresky-MacPherson extension $j_{! *}(-)$ is understood as a functor

$$
\operatorname{Perv}_{\mathcal{S}_{q}^{\text {Conf }}}(\text { Conf }) \rightarrow \operatorname{Perv}_{\mathcal{S}_{q}^{\text {Conf }}}(\text { Conf })
$$

1.6.2. Verdier duality defines a contravariant equivalence

$$
\mathbb{D}^{\text {Verdier }}: \operatorname{Perv}_{\mathcal{S}_{q}^{\text {Conf }}}(\text { Conf }) \simeq \operatorname{Perv}_{\mathcal{G}_{q^{-1}}}(\text { Conf })
$$

where where we note that $\mathcal{G}_{q^{-1}}^{\text {Conf }}$ is the factorization gerbe inverse to $\mathcal{S}_{q}^{\text {Conf }}$.

By construction, we have

$$
\mathbb{D}^{\text {Verdier }}\left(\Omega_{q}^{\text {sml }}\right) \simeq \Omega_{q^{-1}}^{\text {sml }} .
$$

1.6.3. Let $f: X_{1} \rightarrow X_{2}$ be as in Sect. 1.3.7. Let $\Omega_{q, 1}^{\mathrm{sml}}$ (resp., $\Omega_{q, 2}^{\mathrm{sml}}$ ) be the corresponding factorization algebra on Conf $_{1}$ (resp., Conf ${ }_{2}$ ). We obtain that the tautological isomorphism

$$
\stackrel{\circ}{\Omega_{q, 1}^{\mathrm{sml}}} \simeq f_{\text {Conf }}^{*}\left(\stackrel{\circ}{\Omega_{q, 2}^{\mathrm{sml}}}\right)
$$

extends (automatically uniquely) to an isomorphism

$$
\left.\left.\Omega_{q, 1}^{\mathrm{sml}}\right|_{\mathrm{Conf}_{1, e t}} \simeq f_{\mathrm{Conf}}^{*}\left(\Omega_{q, 2}^{\mathrm{sml}}\right)\right|_{\mathrm{Conf}_{1, e t}} .
$$

1.7. Construction of the DeConcini-Kac and Lusztig versions. We remind that we retain the assumption that $q$ is non-degenerate.

\footnotetext{
${ }^{10}$ See also Remark 1.1.6 according to which these factorization algebras may not ultimately be the right objects to consider unless we require a slightly stronger condition, namely, that $q$ avoid small torsion (see Sect. 1.1.5 for what this means).
} 
1.7.1. Recall the notation $\mathfrak{Z}$ (see Sect. 0.5.6), and let $\mathfrak{Z} \hbar$ be its variant with a formal parameter, see Sect. A.3.3

Let $q$ be as in Sect.1.1.1 We define a form $q_{\hbar}$ with values in $\mathfrak{Z}_{\hbar}$ as follows:

Let $q_{\mathbb{Z}}^{\text {min }}$ be the minimal integer-valued $\mathrm{W}$-invariant quadratic form on $\Lambda$ (i.e., one that takes value 1 on the short coroot in every simple factor of our root system). Set

$$
q_{\hbar}=q+q_{\mathbb{Z}}^{\min } \cdot \zeta_{\hbar} ;
$$

where $\zeta_{\hbar} \in \mathfrak{Z}_{\hbar}$ is the element defined as follows:

- For sheaves in the classical topology $\zeta_{\hbar}=\exp (\hbar) \in(\mathrm{e} \llbracket \hbar \rrbracket)^{\times}$;

- For $\ell$-adic sheaves, $\zeta_{\hbar}=1+\hbar \in\left(\mathbb{Z}_{\ell} \llbracket \hbar \rrbracket\right)^{\times}$;

- For D-modules $\zeta=\hbar \in k \llbracket \hbar \rrbracket / \mathbb{Z}$.

Let $\mathcal{G}_{q_{\hbar}}^{\text {Conf }}$ denote the corresponding factorization gerbe on Conf (see Sect. A.3). Explicitly, it can be defined by the same formula as in (1.13), up to replacing $q$ by $q_{\hbar}$.

1.7.2. We will denote the corresponding category of twisted sheaves by $\operatorname{Shv}_{\mathcal{G}_{q \llbracket \hbar \rrbracket}^{\text {Conf }}}($ Conf $)$, and its localization with respect to $\hbar$ by $\operatorname{Shv}_{\mathcal{G}_{q_{((\hbar))} \text { Conf }}}($ Conf $)$, see Sect. A.3.1

We will also consider the ind-completions

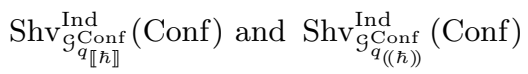

of $\operatorname{Shv}_{\mathcal{G}_{q \llbracket \hbar \rrbracket}^{\text {Conf }}}(\mathrm{Conf})$ and $\operatorname{Shv}_{\mathcal{G}_{q((\hbar))}^{\text {Conf }}}($ Conf $)$, respectively.

We will identify $\operatorname{Shv}_{\substack{\mathcal{G}_{((\hbar))}^{\text {Conf }} \\ \text { Ind }}}\left(\right.$ Conf) with a full subcategory of $\operatorname{Shv}_{\mathcal{G}_{q_{\llbracket \hbar \rrbracket}}^{\text {Ind }}}^{\text {Inf }}$ (Conf) consisting of $\hbar$-local objects.

We let

$$
\operatorname{Perv}(-) \subset \operatorname{Shv}(-) \text { and } \operatorname{Perv}^{\text {Ind }}(-) \subset \operatorname{Shv}^{\text {Ind }}(-)
$$

denote the corresponding abelian subcategories.

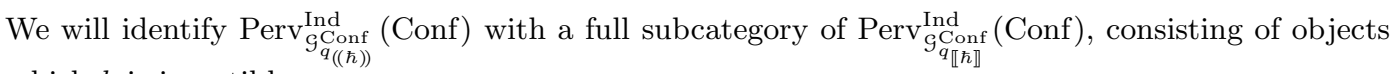
on which $\hbar$ is invertible.

1.7.3. Let $\stackrel{\circ}{\Omega}_{q_{\llbracket \hbar \rrbracket}}$ be the factorization algebra in

$$
\operatorname{Perv}_{\substack{\mathcal{G} \text { Conf } \\ q_{\llbracket \hbar}}}(\text { Conf })
$$

(the latter category identifies with the untwisted $\operatorname{Perv}_{\llbracket \hbar \rrbracket}\left(\mathrm{Conf}^{\circ}\right)$ ), defined as in Sect. 1.5.3

Let

$$
\stackrel{\circ}{\Omega}_{q_{((\hbar))}} \in \operatorname{Perv}_{\mathcal{G}_{((\hbar))}^{\text {Conf }}}(\stackrel{\circ}{\text { Conf })}
$$

be the image of $\stackrel{\circ}{\Omega}_{q_{\llbracket \hbar \rrbracket}}$ under the localization functor

$$
\operatorname{Perv}_{\mathcal{G}_{q \llbracket \hbar \rrbracket}^{\text {Conf }}}(\text { Conf }) \rightarrow \operatorname{Perv}_{\mathcal{S}_{q_{((\hbar))}^{\text {Conf }}}}(\text { Conf }) .
$$

1.7.4. Set

$$
\Omega_{q_{((\hbar))}}:=j_{! *}\left({\stackrel{\circ}{\Omega_{((\hbar))}}}\right) \in \operatorname{Perv}_{\mathcal{G}_{q_{((\hbar))}^{\text {Conf }}}}(\text { Conf }) .
$$

Remark 1.7.5. In many respects, $\Omega_{q_{((\hbar))}}$ behaves similarly to the algebra $\Omega_{q}^{\text {sml }}$ for $q$ which is non-torsion valued. 
1.7.6. Note that the functor

$$
j_{*}: \operatorname{Shv}_{\mathcal{G}_{q}^{\text {Conf }}}(\text { Conf }) \rightarrow \operatorname{Shv}_{\mathcal{G}_{q \llbracket \hbar \rrbracket}^{\text {Conf }}}(\text { Conf })
$$

is t-exact. Indeed, the right t-exactness follows from the right t-exactness of the functor

$$
j_{*}: \operatorname{Shv}_{\mathcal{G}_{q}^{\text {Conf }}}(\stackrel{\circ}{\text { Conf }}) \rightarrow \operatorname{Shv}_{\mathcal{G}_{q}^{\text {Conf }}}(\text { Conf })
$$

(the morphism $j$ is affine), while the left t-exactness follows from the fact that left adjoint of $j_{*}$, i.e., $j^{*}$ is right t-exact.

Consider the object

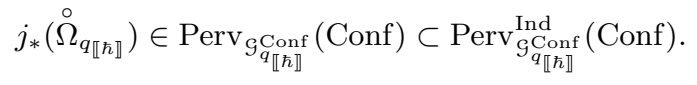

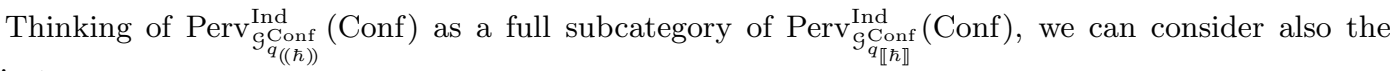
objects

$$
\Omega_{q_{((\hbar))}} \text { and } j_{*}\left(\stackrel{\circ}{\Omega}_{q_{((\hbar))}}\right),
$$

thought of objects of $\operatorname{Perv}_{\mathcal{G}_{q \llbracket \hbar \rrbracket}^{\text {Conf }}}^{\text {Ind }}($ Conf $)$.

We have the injective maps in $\operatorname{Perv}_{\mathcal{G}_{\llbracket \llbracket \hbar \rrbracket}^{\text {Conf }}}^{\text {Ind }}($ Conf $)$

$$
j_{*}\left(\stackrel{\circ}{\Omega}_{q_{\llbracket \hbar \rrbracket}}\right) \rightarrow j_{*}\left(\stackrel{\circ}{\Omega}_{q_{((\hbar))}}\right) \leftarrow \Omega_{q_{((\hbar))}} .
$$

1.7.7. We define the object

$$
\Omega_{q_{\llbracket \hbar \rrbracket}}^{\mathrm{DK}} \in \operatorname{Perv}_{\mathcal{G}_{q_{\llbracket \hbar \rrbracket} \text { Conf }}^{\text {Ind }}}^{\text {Conf })}
$$

as the intersection

$$
j_{*}\left(\stackrel{\circ}{\Omega}_{q_{\llbracket \hbar \rrbracket}}\right) \cap \Omega_{q_{((\hbar))}} \subset j_{*}\left(\stackrel{\circ}{\Omega}_{q_{((\hbar))}}\right) .
$$

By Proposition A.2.7, $\Omega_{q_{\llbracket \hbar \rrbracket}}^{\mathrm{DK}}$ is actually an object of $\operatorname{Perv}_{\mathcal{G}_{q_{\llbracket \hbar \rrbracket} \text { Conf }}}($ Conf $)$.

By construction,

$$
j^{*}\left(\Omega_{q_{\llbracket \hbar \rrbracket}^{\mathrm{DK}}}\right) \simeq \stackrel{\circ}{\Omega}_{q_{\llbracket \hbar \rrbracket}} .
$$

Furthermore, the image of $\Omega_{q_{\llbracket \hbar \rrbracket}}^{\mathrm{DK}}$ in $\operatorname{Perv}_{\mathcal{G}_{q_{((\hbar))} \text { Conf }}}\left(\right.$ Conf) identifies with $\Omega_{q_{((\hbar))}}$.

1.7.8. We define the factorization algebra

$$
\Omega_{q}^{\mathrm{DK}} \in \operatorname{Perv}_{\mathcal{G}_{q}^{\text {Conf }}}(\text { Conf })
$$

as

$$
\Omega_{q_{\llbracket \hbar \rrbracket}}^{\mathrm{DK}} / \hbar \simeq \Omega_{q_{\llbracket \hbar \rrbracket} \mathrm{DK}}^{\mathrm{DK}} \otimes \mathrm{e}
$$

(note that $\Omega_{q_{\llbracket \hbar \rrbracket}}^{\mathrm{DK}}$ is torsion-free (equivalently, flat) over e $\llbracket \hbar \rrbracket$ ).

Finally, we define the factorization algebra

$$
\Omega_{q}^{\text {Lus }} \in \operatorname{Perv}_{\mathcal{G}_{q}^{\text {Conf }}}(\text { Conf })
$$

to be the Verdier dual of $\Omega_{q^{-1}}^{\mathrm{DK}}$.

\section{Properties of the DeConcini-KaC version}

In this section we will formulate a series of theorems pertaining to the behavior of $\Omega_{q}^{\mathrm{DK}}$. These theorems describe (with an increasing degree of precision) how to construct $\Omega_{q}^{\mathrm{DK}}$ inductively, starting from the open locus Conf, and extending across the diagonals.

Throughout this section we will be assuming that $q$ is non-degenerate (see Sect. 1.1.4). 
2.1. First properties of $\Omega_{q}^{\mathrm{DK}}$.

2.1.1. By (1.19), we have

By adjunction, we obtain a map

$$
j^{*}\left(\Omega_{q}^{\mathrm{DK}}\right) \simeq \stackrel{\circ}{\Omega}_{q}
$$

$$
\Omega_{q}^{\mathrm{DK}} \rightarrow j_{*}\left(\stackrel{\circ}{\Omega_{q}}\right) .
$$

Proposition 2.1.2. The map (2.1) is injective.

Proof. The assertion is equivalent to the fact that the quotient

$$
j_{*}\left(\stackrel{\circ}{\Omega}_{q_{\llbracket \hbar \rrbracket}}\right) / \Omega_{q_{\llbracket \hbar \rrbracket}^{\mathrm{DK}}}
$$

is $\hbar$-torsion free. However, the above quotient embeds into

$$
j_{*}\left(\stackrel{\circ}{\Omega}_{q_{((\hbar))}}\right) / \Omega_{q_{((\hbar))}},
$$

on which $\hbar$ is invertible, and the assertion follows.

Remark 2.1.3. When the ground field $k$ has characteristic zero, in Sects. 2.2 2.4, we will give an explicit description of $\Omega_{q}^{\mathrm{DK}}$ as a sub-object of $j_{*}\left(\stackrel{\circ}{\Omega}_{q}\right)$.

2.1.4. From Proposition 2.1 .2 we obtain that there exists an injective map

$$
\Omega_{q}^{\mathrm{sml}} \hookrightarrow \Omega_{q}^{\mathrm{DK}},
$$

extending the identification of the restrictions of both sides to $\stackrel{\circ}{\circ}$ onf with $\stackrel{\circ}{\Omega}$.

We claim:

Theorem 2.1.5. Let $q$ be non-torsion valued. Then the map (2.2) is an isomorphism.

When the ground field $k$ has characteristic 0 , this theorem will be proved in Sect. 3.7 A proof that works for any ground field will be given in Sect. 6.5.6

2.2. Properties of $\Omega_{q}^{\mathrm{DK}}$ in characteristic 0 . From now on, until the end of this section we will assume that the ground field $k$ has characteristic 0 .

We will state a number of properties of $\Omega_{q}^{\mathrm{DK}}$, which will be proved in Sect. 3.7. The factorization algebra $\Omega_{q}^{\mathrm{Lus}}$ will enjoy Verdier-dual properties of those of $\Omega_{q}^{\mathrm{DK}}$.

2.2.1. For $\lambda \in \Lambda^{\text {neg }}$, let $\jmath_{\lambda}$ denote the open embedding complementary to the main diagonal

$$
\Delta_{\lambda}: X \rightarrow X^{\lambda} \text {. }
$$

Note that by Proposition 2.1.2, the tautological map

$$
\left.\Omega_{q}^{\mathrm{DK}}\right|_{\mathrm{Conf}^{\lambda}} \rightarrow\left(\jmath_{\lambda}\right)_{*} \circ\left(\jmath_{\lambda}\right)^{*}\left(\Omega_{q}^{\mathrm{DK}}\right)
$$

induces an injection

$$
\left.\Omega_{q}^{\mathrm{DK}}\right|_{\mathrm{Conf}^{\lambda}} \hookrightarrow H^{0}\left(\left(\jmath_{\lambda}\right)_{*} \circ\left(\jmath_{\lambda}\right)^{*}\left(\Omega_{q}^{\mathrm{DK}}\right)\right),
$$

where $H^{0}$ refers to the perverse t-structure (as it does throughout the paper). Indeed, the composition of (2.4) with the (injective) map

$$
H^{0}\left(\left(\jmath_{\lambda}\right)_{*} \circ\left(\jmath_{\lambda}\right)^{*}\left(\Omega_{q}^{\mathrm{DK}}\right)\right) \rightarrow j_{*} \circ j^{*}\left(\Omega_{q}^{\mathrm{DK}}\right)=j_{*}\left(\stackrel{\circ}{\Omega}_{q}\right)
$$

is the map from Proposition 2.1 .2

This injectivity property is equivalent to the fact that $\left(\Delta_{\lambda}\right)^{!}\left(\Omega_{q}^{\mathrm{DK}}\right)$ lives in (perverse) cohomological degrees $\geq 1$ for $|\lambda|>1$. 
As a consequence of the injectivity of (2.4), we obtain a map

$$
\left.\left(\jmath_{\lambda}\right) !_{*} \circ\left(\jmath_{\lambda}\right)^{*}\left(\Omega_{q}^{\mathrm{DK}}\right) \rightarrow \Omega_{q}^{\mathrm{DK}}\right|_{\mathrm{Conf}^{\lambda}}
$$

which is automatically injective.

2.2.2. We claim:

Theorem 2.2.3. Let $\lambda=w(\rho)-\rho$ with $\ell(w)=2$. Then $\left(\Delta_{\lambda}\right)^{*}\left(\Omega_{q}^{\mathrm{DK}}\right)$ lives in (perverse) cohomological degrees $\leq-1$, i.e., $H^{0}\left(\left(\Delta_{\lambda}\right)^{*}\left(\Omega_{q}^{\mathrm{DK}}\right)\right)=0$.

This theorem will be proved in Sect. 3.7

2.2.4. Let us rephrase Theorem 2.2.3 in terms of the properties of the map (2.5):

Corollary 2.2.5. Let $\lambda=w(\rho)-\rho$ with $\ell(w)=2$. Then the map (2.5) is an isomorphism.

2.3. Inductive construction of $\Omega_{q}^{\mathrm{DK}}$.

2.3.1. From now on, until the end of this section we will impose the following additional assumption on $q$ :

$$
\text { For every positive coroot } \alpha \text { we have the inequality } \operatorname{ord}(q(\alpha)) \geq\langle\rho, \check{\alpha}\rangle=|\check{\alpha}| \text {. }
$$

Note that assumption $\left(^{*}\right)$ reads as "the order of $q(\alpha)$ is large enough". We can rewrite it also as follows: for every simple factor

$$
\operatorname{ord}\left(q\left(\alpha_{0, s}\right)\right) \geq\left|\left(\alpha_{0, s}\right)^{\vee}\right|=: h-1 \text { and } \operatorname{ord}\left(q\left(\alpha_{0, l}\right)\right) \geq\left|\left(\alpha_{0, l}\right)^{\vee}\right|=: h^{\vee}-1,
$$

where $\alpha_{0, s}$ and $\alpha_{0, l}$ ate the longest short and long coroots; $h$ is the Coxeter number of $G$, and $h^{\vee}$ is dual Coxeter number of the Langlands dual $\check{G}$ of $G$

Remark 2.3.2. In what follows we will assume that the result of Geo Theorem 6.4.1], computing the cohomology of the De Concini-Kac quantum group, holds under these assumptions, see Equation (3.27).

In loc.cit., this was established under the assumption that $\operatorname{ord}\left(q\left(\alpha_{0, s}\right)\right)$ is odd (and is not divisible by 3 if $G$ contains a factor isomorphic to $G_{2}$ ). In a recent communication, D. Nakano communicated to the author a proof in the case when $\operatorname{ord}\left(q\left(\alpha_{0, s}\right)\right)$ is even, under a slightly stronger assumption on $\operatorname{ord}\left(q\left(\alpha_{0, s}\right)\right)$.

However, based on the Kazhdan-Lusztig equivalence between quantum groups and the affine algebra, and based on the range of validity of the Kashiwara-Tanisaki localization theorem at the negative level, we believe that $(*)$ is sufficient for the validity of (3.27).

\subsubsection{We claim:}

\section{Theorem 2.3.4.}

(a) Let $\lambda$ be not of the form $w(\rho)-\rho$ with $\ell(w)=2$. Then the object $\left(\Delta_{\lambda}\right)^{!}\left(\Omega_{q}^{\mathrm{DK}}\right)$ lives in (perverse) cohomological degrees $\geq 2$.

(b) Let $\lambda$ be of the form $w(\rho)-\rho$ with $\ell(w)=2$. Then $H^{1}\left(\left(\Delta_{\lambda}\right)^{!}\left(\Omega_{q}^{\mathrm{DK}}\right)\right)$ is isomorphic to $\mathrm{e}_{X}[1]$.

This theorem will be proved in Sect. 3.7

Remark 2.3.5. It is likely that for the validity of Theorem 2.3.4 assumption $\left(^{*}\right)$ is an overkill. What we actually need is that the map (3.13) be an isomorphism, see Remark 3.3.7.

2.3.6. Let us rephrase Theorem 2.3.4 in terms of the maps from Sect. 2.2.1

\section{Corollary 2.3.7.}

(a) Let $\lambda$ be not of the form $w(\rho)-\rho$ with $\ell(w)=2$. Then the map (2.4) is an isomorphism.

(b) Let $\lambda$ be of the form $w(\rho)-\rho$ with $\ell(w)=2$. Then the cone of the map (2.3) is isomorphic to $\left(\Delta_{\lambda}\right) !\left(\mathrm{e}_{X}[1]\right)$; in particular, the object $\left(\jmath_{\lambda}\right)_{*} \circ\left(\jmath_{\lambda}\right)^{*}\left(\Omega_{q}^{\mathrm{DK}}\right)$ is perverse. 
2.3.8. Note that, given the factorization property of $\Omega_{q}^{\mathrm{DK}}$, Corollaries 2.2.5] and 2.3.7 (a) supply a recipe of how to construct $\Omega_{q}^{\mathrm{DK}}$ inductively on $|\lambda|$ :

The base of induction is when $\lambda$ is a negative simple coroot, in which case $\left.\Omega_{q}^{\mathrm{DK}}\right|_{X^{\lambda}}=\mathrm{e}_{X}[1]$. Let now $|\lambda|>1$. By induction and factorization we can assume that we have already constructed $\left.\Omega_{q}^{\mathrm{DK}}\right|_{X^{\lambda}-\Delta_{\lambda}(X)}$. To recover $\left.\Omega_{q}^{\mathrm{DK}}\right|_{X^{\lambda}}$ we proceed as follows:

- If $\lambda=w(\rho)-\rho$ with $\ell(w)=2$, apply $\left.\left(\jmath_{\lambda}\right)\right)_{* *}(-)$;

- If $\lambda$ is not of this form, apply $H^{0}\left(\left(j_{\lambda}\right)_{*}(-)\right)$.

2.4. Further properties of $\Omega_{q}^{\mathrm{DK}}$ in characteristic 0 . We continue to assume that the inequality (*) holds 11

\subsubsection{We claim:}

Theorem 2.4.2.

(a) If $\lambda$ is not of the form $w(\rho)-\rho$ for $w \in W$, then $\left(\Delta_{\lambda}\right)^{!}\left(\Omega_{q}^{\mathrm{DK}}\right)=0$.

(b) If $\lambda$ is the form $w(\rho)-\rho$ for $w \in W$ with $\ell(w) \geq 3$, then $\left(\Delta_{\lambda}\right)^{!}\left(\Omega_{q}^{\mathrm{DK}}\right)$ is isomorphic to $\mathrm{e}_{X}[-\ell(w)+1]$, in particular, it is concentrated in cohomological degree $\ell(w)-2$.

This theorem will be proved in Sect. 3.7

2.4.3. Let us rephrase Theorem 2.4.2(a) in terms of properties of the map (2.3):

Corollary 2.4.4. If $\lambda$ is not of the form $w(\rho)-\rho$ for $w \in W$, then the map (2.3) is an isomorphism; in particular, $\left(\jmath_{\lambda}\right)_{*} \circ\left(\jmath_{\lambda}\right)^{*}\left(\Omega_{q}^{\mathrm{DK}}\right)$ is perverse.

2.4.5. Assume for a moment that $q$ is non-torsion-valued, so that by Theorem 2.1.5 the map

$$
\Omega^{\mathrm{sml}} \rightarrow \Omega^{\mathrm{DK}}
$$

is an isomorphism, and by duality, the map

$$
\Omega^{\mathrm{Lus}} \rightarrow \Omega^{\mathrm{sml}}
$$

is also an isomorphism.

From Corollary 2.4.4 we obtain:

Corollary 2.4.6. Let $q$ be non-torsion-valued. Then for any $\lambda \in \Lambda^{\text {neg }}-0$ not of the form $w(\rho)-\rho$, both restrictions $\Delta_{\lambda}^{!}\left(\Omega_{q}^{\mathrm{sml}}\right)$ and $\Delta_{\lambda}^{*}\left(\Omega_{q}^{\mathrm{sml}}\right)$ vanish.

Remark 2.4.7. Note that in the case of non-torsion valued $q$, Theorem 2.1.5 implies that the map (2.5) is an isomorphism for all $\lambda$.

In particular, in this case, for $\lambda$ not of the form $w(\rho)-\rho$ with $w \in W$, all the maps in

$$
\begin{aligned}
\left.\left.\Omega^{\mathrm{sml}}\right|_{\mathrm{Conf}^{\lambda}} \simeq \Omega^{\mathrm{DK}}\right|_{\mathrm{Conf}^{\lambda}} \simeq\left(\jmath_{\lambda}\right)_{! *} \circ\left(\jmath_{\lambda}\right)^{*}\left(\Omega_{q}^{\mathrm{DK}}\right) & \left.\rightarrow \Omega_{q}^{\mathrm{DK}}\right|_{\mathrm{Conf}^{\lambda}} \rightarrow \\
& \rightarrow H^{0}\left(\left(\jmath_{\lambda}\right)_{*} \circ\left(\jmath_{\lambda}\right)^{*}\left(\Omega_{q}^{\mathrm{DK}}\right)\right) \rightarrow\left(\jmath_{\lambda}\right)_{*} \circ\left(\jmath_{\lambda}\right)^{*}\left(\Omega_{q}^{\mathrm{DK}}\right)
\end{aligned}
$$

are isomorphisms. Indeed, the fact that the composite arrow is an isomorphism is equivalent to the vanishing of $\left(\Delta_{\lambda}\right)^{!}\left(\Omega^{\mathrm{sml}}\right)$, which is the content of Corollary 2.4.6.

2.5. A sharper estimate. In this subsection we will assume that the inequality in $(*)$ is sharp.

2.5.1. We claim:

Theorem 2.5.2. For $\lambda$ of the form $w(\rho)-\rho$ for $w \in W$ with $\ell(w) \geq 3$, the object $\left(\Delta_{\lambda}\right)^{*}\left(\Omega_{q}^{\mathrm{DK}}\right)$ is concentrated in cohomological degrees $\leq-1$.

This theorem will be proved in Sect. 3.7.

\footnotetext{
${ }^{11}$ Unlike the case of Theorem 2.3 .4 it is likely that for the validity of Theorem 2.4 .2 below, the full strength of $(*)$ is needed.
} 
2.5.3. Let us rephrase Theorem 2.5.2 in terms of properties of the map (2.5):

Corollary 2.5.4. For $\lambda$ of the form $w(\rho)-\rho$ for $w \in W$ with $\ell(w) \geq 3$, the map (2.5) is an isomorphism.

Remark 2.5.5. Thus, we obtain that if the inequality in $\left(^{*}\right)$ is sharp, for $\lambda$ of the form $w(\rho)-\rho$ for $w \in W$ with $\ell(w) \geq 3$, both maps

$$
\left.\left(\jmath_{\lambda}\right) !_{*} \circ\left(\jmath_{\lambda}\right)^{*}\left(\Omega_{q}^{\mathrm{DK}}\right) \rightarrow \Omega_{q}^{\mathrm{DK}}\right|_{\mathrm{Conf}^{\lambda}} \rightarrow H^{0}\left(\left(\jmath_{\lambda}\right)_{*} \circ\left(\jmath_{\lambda}\right)^{*}\left(\Omega_{q}^{\mathrm{DK}}\right)\right)
$$

are isomorphisms.

2.5.6. The above Theorems 2.4 .2 and 2.5 .2 give an even more detailed recipe for the inductive construction of $\Omega_{q}^{\mathrm{DK}}$ (cf. Sect. 2.3.8). By induction and factorization we can assume that we have already constructed $\left.\Omega_{q}^{\mathrm{DK}}\right|_{X^{\lambda}-\Delta_{\lambda}(X)}$. To recover $\left.\Omega_{q}^{\mathrm{DK}}\right|_{X^{\lambda}}$ we proceed as follows:

- If $\lambda=w(\rho)-\rho$ with $\ell(w)=2$, apply $\left(j_{\lambda}\right) ! *(-)$;

- If $\lambda=w(\rho)-\rho$ with $\ell(w) \geq 3$, apply $H^{0}\left(\left(j_{\lambda}\right)_{*}(-)\right)$, which is the same as $\left(j_{\lambda}\right) ! *(-)$;

- If $\lambda$ is not of the form $w(\rho)-\rho$, apply $H^{0}\left(\left(j_{\lambda}\right)_{*}(-)\right)$, which is the same as $\left(\jmath_{\lambda}\right)_{*}(-)$ (if $q$ is non-torsion valued, this is also the same as $\left.\left(j_{\lambda}\right) ! *(-)\right)$.

\section{FaCtorization algebras Via QuANtum Groups}

In this section we will work with constructible sheaves in the classical topology with coefficients in a field e (assumed algebraically closed and of characteristic 0 ).

We will describe a relationship between the (three versions of the) factorization algebra $\Omega_{q}$ and the corresponding versions of the quantum group.

\subsection{Quantum Hopf algebras.}

3.1.1. Let Vect $^{\Lambda}$ denote the category of $\Lambda$-graded vector spaces, which is the same as the category $\operatorname{Rep}(\check{T})$, of algebraic representations of the torus $\check{T}$.

For $\lambda \in \Lambda$ we denote by $\mathrm{e}^{\lambda}$ the corresponding object of $\operatorname{Vect}^{\Lambda}$ : this is a copy of e placed in coweight component $\lambda$.

For an on object $V \in \operatorname{Vect}^{\Lambda}$ and $\lambda \in \Lambda$, we will let $(V)^{\lambda}$ denote its $\lambda$-coweight component, i.e.,

$$
(V)^{\lambda}=\mathcal{H}_{o m_{\text {Vect }}}\left(\mathrm{e}^{\lambda}, V\right) .
$$

3.1.2. Choose a bilinear form

$$
b^{\prime}: \Lambda \otimes \Lambda \rightarrow \mathrm{e}^{\times}
$$

so that $q(\lambda)=b^{\prime}(\lambda, \lambda)$.

Such a form $b^{\prime}$ defines a braiding on

$$
\operatorname{Rep}(\check{T}) \simeq \operatorname{Vect}^{\Lambda}
$$

viewed as a monoidal category. Explicitly, the braiding automorphism

$$
\mathrm{e}^{\lambda+\mu}=\mathrm{e}^{\lambda} \otimes \mathrm{e}^{\mu} \stackrel{R}{\rightarrow} \mathrm{e}^{\mu} \otimes \mathrm{e}^{\lambda}=\mathrm{e}^{\mu+\lambda}
$$

is given by multiplication by $b^{\prime}(\lambda, \mu)$.

Denote the resulting braided monoidal category by $\operatorname{Rep}_{q}(\check{T})$.

Remark 3.1.3. It follows from GLys1, Sects. C.2.3] that $\operatorname{Rep}_{q}(\check{T})$, viewed as a braided monoidal category, depends only on $q$ (and not the choice of $b^{\prime}$ ), up to a canonical equivalence. A choice of $b^{\prime}$ serves to identify the underlying monoidal category with $\operatorname{Rep}(\check{T})$. 
3.1.4. We will consider a certain type of Hopf algebras in $\operatorname{Rep}_{q}(\check{T})$. Namely, we will impose the following conditions on our Hopf algebra (denoted $H$ ):

- All weight components $H^{\lambda}$ lie in the heart of the t-structure and are finite-dimensional;

- If the weight component $H^{\lambda}$ is non-zero, then $\lambda \in \Lambda^{\text {pos }}$;

- The unit map $1: \mathrm{e} \rightarrow H^{0}$ is an isomorphism.

3.1.5. We note that component-wise duality assigns to such a Hopf algebra $H$ its dual, to be denoted $H^{\vee}$, which is naturally a Hopf algebra in $\operatorname{Rep}_{q^{-1}}(\check{T})$;

$$
\left(H^{\vee}\right)^{\lambda}=\left(H^{\lambda}\right)^{\vee} \text {. }
$$

3.2. The free, cofree and small versions of the positive part of the quantum group. Throughout this subsection we will be assuming that $q$ is non-degenerate (see Sect. 1.1.4 for what this means) 12 .

We will consider some particular Hopf algebras in $\operatorname{Rep}_{q}(\check{T})$, attached to our root system.

3.2.1. The first one is the free algebra, denoted $U_{q}^{\text {free }}(\check{N})$. As an associative algebra, it is the free associative algebra on the object

$$
\bigoplus_{i} \mathrm{e}^{\alpha_{i}} \in \operatorname{Rep}_{q}(\check{T})
$$

In other words, it is defined by the universal property that

$$
\operatorname{Hom}_{\operatorname{Ass} A l g\left(\operatorname{Rep}_{q}(\check{T})\right)}\left(U_{q}^{\text {free }}(\check{N}), A\right)=\operatorname{Hom}_{\operatorname{Rep}_{q}(\check{T})}\left(\oplus_{i} \mathrm{e}^{\alpha_{i}}, A\right) \simeq \prod_{i}(A)^{\alpha_{i}} .
$$

In particular, we have the canonical maps

$$
e_{i}: \mathrm{e}^{\alpha_{i}} \rightarrow U_{q}^{\text {free }}(\check{N})
$$

which we will refer to as the "generators".

The Hopf algebra structure on $U_{q}^{\text {free }}(\check{N})$ is determined by the requirement that the co-multiplication map

$$
\text { comult }: U_{q}^{\text {free }}(\check{N}) \rightarrow U_{q}^{\text {free }}(\check{N}) \otimes U_{q}^{\text {free }}(\check{N}),
$$

viewed as a map in $\operatorname{Ass} A \lg \left(\operatorname{Rep}_{q}(\check{T})\right)$, corresponds under (3.1) to the maps

$$
e_{i} \otimes 1+1 \otimes e_{i}: \mathrm{e}^{\alpha_{i}} \rightarrow U_{q}^{\text {free }}(\check{N}) \otimes U_{q}^{\text {free }}(\check{N}),
$$

where 1 stands for the unit map e $\rightarrow U_{q}^{\text {free }}(\check{N})$.

3.2.2. We set $U_{q}^{\text {cofree }}(\check{N})$ to be dual (in the sense of Sect. 3.1.5) of the Hopf algebra $U_{q^{-1}}^{\text {free }}(\check{N})$ in $\operatorname{Rep}_{q^{-1}}(\check{T})$.

As a co-associative co-algebra it has the universal property that

$$
\operatorname{Hom}_{\mathrm{CoAss} \operatorname{CoAlg}\left(\operatorname{Rep}_{q}(\check{T})\right)}\left(A, U_{q}^{\text {cofree }}(\check{N})\right)=\operatorname{Hom}_{\operatorname{Rep}_{q}(\check{T})}\left(A, \mathrm{e}^{\alpha_{i}}\right) \simeq \prod_{i}\left((A)^{\alpha_{i}}\right)^{\vee} .
$$

In particular, we have the canonical maps

$$
e_{i}^{\vee}: U_{q}^{\text {cofree }}(\check{N}) \rightarrow \mathrm{e}^{\alpha_{i}} .
$$

The Hopf algebra structure on $U_{q}^{\text {cofree }}(\check{N})$ is determined by the requirement that the multiplication map

$$
\text { mult }: U_{q}^{\text {cofree }}(\check{N}) \otimes U_{q}^{\text {cofree }}(\check{N}) \rightarrow U_{q}^{\text {cofree }}(\check{N})
$$

corresponds under (3.3) to the maps

$$
e_{i}^{\vee} \otimes 1^{\vee}+1^{\vee} \otimes e_{i}^{\vee}: U_{q}^{\text {cofree }}(\check{N}) \otimes U_{q}^{\text {cofree }}(\check{N}) \rightarrow \mathrm{e}^{\alpha_{i}},
$$

where $1^{\vee}$ denotes the co-unit map

$$
U_{q}^{\text {cofree }}(\check{N}) \rightarrow \text { e. }
$$

\footnotetext{
${ }^{12}$ See, however, Remark 1.1.6
} 
3.2.3. It is easy to see that the map $e_{i}$ of (3.2) defines an isomorphism of $\alpha_{i}$-coweight components. We let $e_{i}^{\vee}$ denote the resulting map

$$
U_{q}^{\text {free }}(\check{N}) \rightarrow \mathrm{e}^{\alpha_{i}},
$$

the projection onto the $\alpha_{i}$-coweight component.

We have a commutative diagram

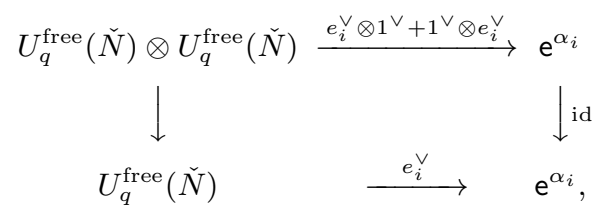

where $1^{\vee}$ denotes the co-unit map $U_{q}^{\text {free }}(\check{N}) \rightarrow$ e.

Similarly, it is easy to see that the map $e_{i}^{\vee}$ of (3.4) defines isomorphisms on $\alpha_{i}$-coweight components. In particular, we have the canonical map

$$
e_{i}: \mathrm{e}^{\alpha_{i}} \rightarrow U_{q}^{\text {cofree }}(\check{N})
$$

that makes the diagrams

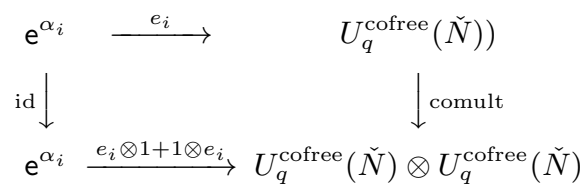

commute.

3.2.4. We now claim that there exists a canonically defined map of Hopf algebras

$$
U_{q}^{\text {free }}(\check{N}) \rightarrow U_{q}^{\text {cofree }}(\check{N})
$$

As a map of associative algebras, (3.7) is determined by the requirement that the diagrams

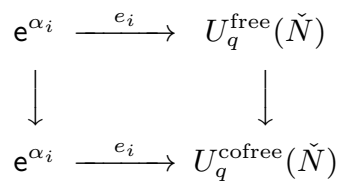

commute.

The compatibility with the coalgebra structure is insured by the diagrams (3.6).

3.2.5. It follows that in terms of (3.3), the map (3.7) corresponds to the maps $e_{i}^{\vee}$ of (3.5).

We obtain that the duality functor of Sect. 3.1.5 sends the map (3.7) to the map

$$
U_{q^{-1}}^{\text {free }}(\check{N}) \rightarrow U_{q^{-1}}^{\text {cofree }}(\check{N})
$$

in $\operatorname{Rep}_{q^{-1}}(\check{T})$.

3.2.6. Let $u_{q}(\check{N})$ be the Hopf algebra in $\operatorname{Rep}_{q}(\check{T})$ equal to the image of the map (3.7).

This is the small version of (the positive part of) the quantum group. 
3.2.7. Recall that for a pair of vertices of the Dynkin diagram $i, j$ one can attach a canonical element

$$
S_{q}^{i, j} \in U_{q}^{\text {free }}(\check{N})
$$

called the quantum Serre relation, whose coweight is

$$
\alpha_{i}+\left(1-\left\langle\alpha_{i}, \check{\alpha}_{j}\right\rangle\right) \in \Lambda^{\mathrm{pos}}
$$

We normalize $S_{q}^{i, j}$ so that it has the form

$$
e_{i}^{1-\left\langle\alpha_{i}, \check{\alpha}_{j}\right\rangle} \cdot e_{j}+\ldots+e_{j} \cdot e_{i}^{1-\left\langle\alpha_{i}, \check{\alpha}_{j}\right\rangle},
$$

The following is established in [Ro]:

Lemma 3.2.8. Let $q$ be non-torsion valued. Then $u_{q}(\check{N})$ is the quotient of $U_{q}^{\text {free }}(\check{N})$ by the two-sided ideal $\mathcal{J}_{q}$ generated by the elements $S_{q}^{i, j}$ for all pairs of vertices $i, j \in I$.

3.3. The DeConcini-Kac and Luztig's versions of the positive part of the quantum group. In order to define the Hopf algebras $U_{q}^{\mathrm{DK}}(\check{N})$ and $U_{q}^{\mathrm{Lus}}(\check{N})$, we will need to consider the deformation of $q$ as in Sect. 1.7

3.3.1. Recall the quadratic form

$$
q_{\hbar}: \Lambda \rightarrow(\mathrm{e} \llbracket \hbar \rrbracket)^{\times} .
$$

We introduce the category $\operatorname{Rep}_{q_{\llbracket \hbar \rrbracket}}(\check{T})$ and its localization $\operatorname{Rep}_{q_{((\hbar))}}(\check{T})$ by the recipe of Sect. A.1.1 applied to the small category

$$
\operatorname{Rep}(\check{T})^{\text {loc.fin.dim. }}=\left(\operatorname{Vect}^{\text {fin.dim. }}\right)^{\Lambda}
$$

where the superscript "loc.fin.dim." means that we are considering representations with each weight component finite-dimensional.

The procedure of Sect. A.2.1 endows these categories with a t-structure, so that the analog of Proposition A.2.2 holds.

Note that as abstract DG categories, we have:

$$
\operatorname{Rep}_{q_{\llbracket \hbar \rrbracket}}(\check{T}) \simeq\left((\mathrm{e} \llbracket \hbar \rrbracket-\bmod )^{\mathrm{f} . \mathrm{g} \cdot}\right)^{\Lambda} \text { and } \operatorname{Rep}_{q_{((\hbar))}}(\check{T}) \simeq\left(\left(\operatorname{Vect}_{\mathrm{e}((\hbar))}\right)^{\mathrm{fin} \cdot \operatorname{dim} \cdot}\right)^{\Lambda},
$$

respectively, equipped with their natural t-structures.

The role of $q_{\hbar}$ is that it defines a braided structure on these categories. In particular, we obtain

$$
\operatorname{Rep}_{q_{((\hbar))}}(\check{T}) \simeq \operatorname{Rep}_{q^{\prime}}(\check{T})^{\text {loc.fin.dim. }}
$$

where the latter is the variant of the category $\operatorname{Rep}_{q^{\prime}}(\check{T})$ over the field $\mathrm{e}((\hbar))$ with $q^{\prime}=q_{\hbar}$.

3.3.2. Consider the resulting Hopf algebras in $\operatorname{Rep}_{q_{((\hbar))}}(\check{T})$ :

$$
U_{q_{((\hbar))}}^{\text {free }}(\check{N}) \text { and } U_{q_{((\hbar))}}^{\text {cofree }}(\check{N})
$$

and the map

$$
U_{q_{((\hbar))}}^{\text {free }}(\check{N}) \rightarrow U_{q_{((\hbar))}}^{\text {cofree }}(\check{N}) .
$$

Set $U_{q_{((\hbar))}}(\check{N})$ be the image of the map (3.8). As in Lemma 3.2.8 the kernel $\mathcal{J}_{q_{((\hbar))}}$ of the projection

$$
U_{q_{((\hbar))}}^{\text {free }}(\check{N}) \rightarrow U_{q_{((\hbar))}}(\check{N})
$$

is generated by the corresponding elements

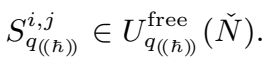


3.3.3. Consider now the Hopf algebra $U_{q_{\llbracket \hbar \rrbracket}}^{\text {free }}(\check{N})$ in $\operatorname{Rep}_{q_{\llbracket \hbar \rrbracket}}(\check{T})$, and set

$$
\mathcal{J}_{q_{\llbracket \hbar \rrbracket}}:=U_{q_{\llbracket \hbar \rrbracket}}^{\text {free }}(\check{N}) \cap \mathcal{J}_{q_{((\hbar))}},
$$

where the intersection is taking place in $U_{q_{((\hbar))}}^{\text {free }}(\check{N})$.

Then $\mathcal{J}_{q_{\llbracket \hbar \rrbracket}} \subset U_{q_{\llbracket \hbar \rrbracket}}^{\text {free }}(\check{N})$ is a Hopf ideal, and we define

$$
U_{q_{\llbracket \hbar \rrbracket} \mathrm{DK}}^{\mathrm{DK}}(\check{N}):=U_{q_{\llbracket \hbar \rrbracket}}^{\text {free }}(\check{N}) / \mathcal{J}_{q_{\llbracket \hbar \rrbracket}} .
$$

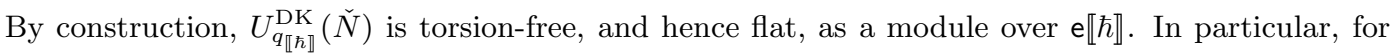
every $\lambda \in \Lambda$, the corresponding coweight component

$$
\left(U_{q_{\llbracket \hbar \rrbracket}^{\mathrm{DK}}}^{\mathrm{D}}(\check{N})\right)^{\lambda}
$$

is a free module of finite rank over $e \llbracket \hbar \rrbracket$. Moreover this rank equals

$$
\operatorname{dim}_{\mathrm{e}((\hbar))}\left(\left(U_{q_{((\hbar))}^{\mathrm{DK}}}^{\mathrm{DK}}(\check{N})\right)^{\lambda}\right)=\operatorname{dim}_{\mathrm{e}}\left(U(\check{\mathfrak{n}})^{\lambda}\right),
$$

where $U(\check{\mathfrak{n}})$ is the usual universal enveloping algebra of $\check{\mathfrak{n}}$.

3.3.4. The map

$$
U_{q_{\llbracket \hbar \rrbracket}}^{\text {free }}(\check{N}) \rightarrow U_{q_{\llbracket \hbar \rrbracket} \text { cofree }}(\check{N})
$$

factors as

$$
U_{q_{\llbracket \hbar \rrbracket}}^{\text {free }}(\check{N}) \rightarrow U_{q_{\llbracket \hbar \rrbracket}}^{\mathrm{DK}}(\check{N}) \rightarrow U_{q_{\llbracket \hbar \rrbracket}}^{\text {cofree }}(\check{N})
$$

Note that the map

$$
U_{q_{\llbracket \hbar \rrbracket}^{\mathrm{DK}}}^{\mathrm{DK}}(\check{N}) \rightarrow U_{q_{\llbracket \hbar \rrbracket}}^{\text {cofree }}(\check{N})
$$

is injective, but its cokernel has $\hbar$-torsion, so it ceases to be injective $\bmod \hbar$.

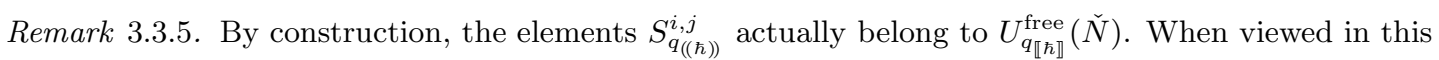
capacity, we will denote them by $S_{q_{\llbracket \hbar \rrbracket}}^{i, j}$, and they belong to the ideal $\mathcal{J}_{q_{\llbracket \hbar \rrbracket}}$.

In Sect. 3.7.6 we will show that under the assumption that $q$ satisfies $\left(^{*}\right.$ ) (see Sect. 2.3.1) the elements $S_{q_{\llbracket \hbar \rrbracket}^{i, j}}$ generate $\mathcal{J}_{q_{\llbracket \hbar \rrbracket}}$ as a two-sided ideal.

Hence, in this case, we can explicitly write $U_{q_{\llbracket \hbar \rrbracket} \mathrm{DK}}(\check{N})$ as the quotient of $U_{q_{\llbracket \hbar \rrbracket}}^{\text {free }}(\check{N})$ by the elements $S_{q_{\llbracket \hbar \rrbracket}^{i, j}}$.

3.3.6. Define

$$
U_{q}^{\mathrm{DK}}(\check{N}):=U_{q_{\llbracket \hbar \rrbracket} \mathrm{DK}}^{\mathrm{DK}}(\check{N}) / \hbar \simeq U_{q_{\llbracket \hbar \rrbracket}^{\mathrm{DK}}}^{\mathrm{D}}(\check{N}) \underset{\mathrm{e \llbracket \hbar \rrbracket}}{\otimes} \mathrm{e},
$$

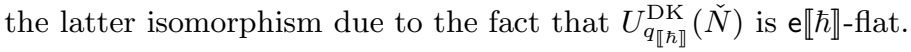

The factorization (3.10) implies that the maps

$$
U_{q}^{\text {free }}(\check{N}) \rightarrow u_{q}(\check{N}) \hookrightarrow U_{q}^{\text {cofree }}(\check{N})
$$

factor as

$$
U_{q}^{\text {free }}(\check{N}) \rightarrow U_{q}^{\mathrm{DK}}(\check{N}) \rightarrow u_{q}(\check{N}) \hookrightarrow U_{q}^{\text {cofree }}(\check{N}) .
$$

Remark 3.3.7. From Remark 3.3.5 we obtain that under the assumption that $q$ satisfies $(*), U_{q}^{\text {DK }}(\check{N})$ equals the quotient of $U_{q}^{\text {free }}(\check{N})$ by the two-sided ideal generated by the elements $S_{q}^{i, j}$.

In general, for a given value of $q$, the map

$$
U_{q}^{\text {free }}(\check{N}) /\left\langle S_{q}^{i, j}\right\rangle \rightarrow U_{q}^{\mathrm{DK}}(\check{N})
$$

is an isomorphism if and and only if $U_{q}^{\text {free }}(\check{N}) /\left\langle S_{q}^{i, j}\right\rangle$ has the right size, i.e., if

$$
\operatorname{dim}\left(\left(U_{q}^{\text {free }}(\check{N}) /\left\langle S_{q}^{i, j}\right\rangle\right)^{\lambda}\right)=\operatorname{dim}\left(U(\check{\mathfrak{n}})^{\lambda}\right), \quad \lambda \in \Lambda^{\text {pos }}
$$


3.3.8. As a (trivial) particular case, from Lemma 3.2 .8 we obtain:

Corollary 3.3.9. Let $q$ be non-torsion valued. Then the map

$$
U_{q}^{\mathrm{DK}}(\check{N}) \rightarrow u_{q}(\check{N})
$$

is an isomorphism.

3.3.10. We define the Hopf algebra $U_{q}^{\text {Lus }}(\check{N})$ as the dual (in the sense of Sect. 3.1.5) of the Hopf algebra $U_{q^{-1}}^{\mathrm{DK}}(\check{N})$ in $\operatorname{Rep}_{q^{-1}}(\check{T})$.

By duality, from (3.12) we obtain that the maps in (3.11) factor also as

$$
U_{q}^{\text {free }}(\check{N}) \rightarrow u_{q}(\check{N}) \hookrightarrow U_{q}^{\text {Lus }}(\check{N}) \hookrightarrow U_{q}^{\text {cofree }}(\check{N})
$$

To summarize we have the following string of maps of Hopf algebras

$$
U_{q}^{\text {free }}(\check{N}) \rightarrow U_{q}^{\text {DK }}(\check{N}) \rightarrow u_{q}(\check{N}) \hookrightarrow U_{q}^{\text {Lus }}(\check{N}) \hookrightarrow U_{q}^{\text {cofree }}(\check{N})
$$

3.3.11. Finally, from Corollary 3.3 .9 we obtain:

Corollary 3.3.12. Let $q$ be non-torsion valued. Then both maps

$$
U_{q}^{\mathrm{DK}}(\check{N}) \rightarrow u_{q}(\check{N}) \text { and } u_{q}(\check{N}) \hookrightarrow U_{q}^{\mathrm{Lus}}(\check{N})
$$

are isomorphisms.

3.4. From Hopf algebras to factorization algebras. In this subsection we summarize the construction from GLys2, Sect. 29.5].

3.4.1. Let $X$ be $\mathbb{A}^{1}$ with a chosen coordinate. Let $\mathcal{G}_{q}^{\text {Conf }}$ be the $\mathrm{e}^{\times}$-gerbe attached to the quadratic form $q: \Lambda \rightarrow \mathrm{e}^{\times}$.

Note that, according to formula (1.14), our choice of the coordinate on $\mathbb{A}^{1}$ gives rise to a trivialization of the gerbe $\mathcal{G}_{q}^{\lambda}$ for every $\lambda \in \Lambda^{\text {neg }}-0$.

3.4.2. The construction of GLys2, Sect. 29.5] defines a contravariant equivalence between the category of Hopf algebras in $\operatorname{Rep}_{q}(\check{T})$, satisfying the conditions of Sect. 3.1.4 and that of factorization algebras in $\operatorname{Perv}_{\mathcal{G}_{q}^{\text {Conf }}}($ Conf)

$$
H \mapsto \Omega_{H},
$$

which has the following properties.

3.4.3. For $\lambda \in \Lambda^{\text {neg }}-0$, let $\iota_{\lambda}$ denote the embedding of the point $\lambda \cdot 0$ into Conf $^{\lambda}$, cf. Sect. 1.2.5 (Note that the above trivialization of the gerbe $\mathcal{G}_{q}^{\lambda}$ allows to view fibers and cofibers of objects of $\operatorname{Shv}_{\mathcal{G}_{q}^{\text {Conf }}}($ Conf $)$ at $\lambda \cdot 0 \in \operatorname{Conf}^{\lambda}$ as objects of Vect.)

Let $H-\bmod \left(\operatorname{Rep}_{q}(\check{T})\right)$ denote the category of left $H$-modules in $\operatorname{Rep}_{q}(\check{T})$, where $H$ is viewed as an associative algebra. Note that the operation of tensor product on the right defines an action of $\operatorname{Rep}_{q}(\check{T})$ on $H-\bmod \left(\operatorname{Rep}_{q}(\check{T})\right)$,

$$
\mathcal{M}, \mathrm{e}^{\lambda} \mapsto \mathcal{M} \otimes \mathrm{e}^{\lambda}
$$

For $\mathcal{M}_{1}, \mathcal{M}_{2} \in H$-mod, let $\underline{\mathcal{H} o m}_{H}\left(\mathcal{M}_{1}, \mathcal{M}_{2}\right)$ denote the $\Lambda$-graded vector space whose $\lambda$-component is

$$
\mathcal{H} o m_{H-\bmod \left(\operatorname{Rep}_{q}(\check{T})\right)}\left(\mathcal{M}_{1} \otimes \mathrm{e}^{\lambda}, \mathcal{M}_{2}\right) .
$$

3.4.4. The first property of the functor 3.15) says that

$$
\iota_{\lambda}^{\prime}\left(\Omega_{H}\right) \simeq\left({\underline{\mathcal{H} O m_{H}}}_{H}(\mathrm{e}, \mathrm{e})\right)^{\lambda} .
$$

Note that the RHS in (3.16) identifies canonically with the $\lambda$-component of the cochain complex of $H$,

$$
\mathrm{C} \cdot(H) \text {, }
$$

where $H$ is viewed as a plain $\Lambda^{\text {pos }}$-graded associative augmented algebra via the (monoidal!) functor

$$
\operatorname{Rep}_{q}(\check{T}) \rightarrow \operatorname{Rep}(\check{T})=\operatorname{Vect}^{\Lambda} \rightarrow \operatorname{Vect} .
$$


3.4.5. The second property of the functor (3.15) states that we have a canonical isomorphism (functorial in $H)$ :

$$
\mathbb{D}^{\text {Verdier }}\left(\Omega_{H}\right) \simeq \Omega_{H^{\vee}}
$$

where both sides are viewed as factorization algebras in $\operatorname{Perv}_{\mathcal{G}_{q^{-1}}^{\Lambda}}($ Conf $)$.

3.4.6. Combining with (3.16) we obtain that the following expression for the ${ }^{*}$-fibers of $\Omega(H)$ :

$$
\iota_{\lambda}^{*}\left(\Omega_{H}\right) \simeq\left(\left(\underline{\mathcal{H} o m}_{H^{\vee}}(\mathrm{e}, \mathrm{e})\right)^{\lambda}\right)^{\vee} \simeq\left(\left(\mathrm{C} \cdot\left(H^{\vee}\right)\right)^{\lambda}\right)^{\vee} \simeq\left(\mathrm{C} \cdot\left(H^{\vee}\right)\right)^{-\lambda},
$$

where C.(-) stands for the chain complex of a given associative augmented algebra.

3.4.7. For a given $\lambda \in \Lambda^{\text {neg }}-0$, let

$$
\left(\iota_{\lambda}\right)^{! *}: \operatorname{Shv}_{\mathcal{G}_{q} \operatorname{Conf}}(\mathrm{Conf}) \rightarrow \text { Vect }
$$

be the functor of hyperbolic restriction along $\iota_{\lambda}$. This functor is a feature of the topological setting, and it is defined as the composite of the following two functors:

The first functor is *-restriction along

$$
\operatorname{Conf}_{\mathbb{R}} \hookrightarrow \text { Conf, }
$$

where $\operatorname{Conf}_{\mathbb{R}}$ is the topological submanifold that corresponds to real configurations, i.e., we take points

$$
\sum_{k} \lambda_{k} \cdot x_{k} \in \text { Conf }
$$

where all $x_{k}$ belong to $\mathbb{R} \subset \mathbb{C}=\mathbb{A}^{1}$.

The second functor is !-restriction along

$$
\mathrm{pt} \rightarrow \operatorname{Conf}_{\mathbb{R}}
$$

corresponding to the point $\lambda \cdot 0$.

Note that the functor $\left(\iota_{\lambda}\right)^{! *}$, when applied to the full subcategory of $\operatorname{Shv}_{\mathcal{G}}\left(\operatorname{Conf}^{\lambda}\right)$, consisting of objects constructible with respect to the diagonal stratification, is t-exact and conservative.

3.4.8. The third property of the functor (3.15) states that we have a canonical isomorphism

$$
\left(\iota_{\lambda}\right)^{! *}\left(\Omega_{H}\right) \simeq\left(H^{-\lambda}\right)^{\vee} .
$$

3.4.9. The isomorphism (3.19), combined with the properties of $\left(\iota_{\lambda}\right)^{! *}$ implies:

Corollary 3.4.10. If a map of Hopf algebras $H_{1} \rightarrow H_{2}$ is injective/surjective, then the corresponding map in $\operatorname{Perv}_{\mathcal{G}_{q}^{\text {Conf }}}($ Conf)

$$
\Omega_{H_{2}} \rightarrow \Omega_{H_{1}}
$$

is surjective/injective.

3.5. Factorization algebras attached to quantum groups. In this subsection we will apply the functor (3.15) to the Hopf algebras associated with quantum groups from Sects. 3.2 and 3.3

In particular, we will again impose the assumption that $q$ avoids small torsion. 
3.5.1. Denote

$$
\begin{gathered}
\Omega_{q, \text { Quant }}^{\text {free }}:=\Omega_{U_{q}^{\text {free }(\check{N})}}, \Omega_{q, \text { Quant }}^{\text {cofree }}:=\Omega_{U_{q}^{\text {cofree }}(\check{N})}, \\
\Omega_{q, \text { Quant }}^{\text {sml }}:=\Omega_{u_{q}(\check{N})}, \Omega_{q, \text { Quant }}^{\text {DK }}:=\Omega_{U_{q}^{\text {DK }}(\check{N})}, \Omega_{q, \text { Quant }}^{\text {Lus }}:=\Omega_{U_{q}^{\text {Lus }(\check{N})}} .
\end{gathered}
$$

First, we claim:

Proposition 3.5.2. There exists a canonical identification

$$
\Omega_{q, \text { Quant }}^{\text {free }} \simeq j_{*}\left(\stackrel{\circ}{\Omega_{q}}\right)
$$

as factorization algebras.

Proof. First we establish an identification

$$
j^{*}\left(\Omega_{q, \text { Quant }}^{\text {free }}\right) \simeq \stackrel{\circ}{\Omega}_{q} .
$$

By factorization, it is enough to establish the isomorphisms

$$
\left.\Omega_{q, \text { Quant }}^{\text {free }}\right|_{\text {Conf }^{-\alpha_{i}}} \simeq \mathrm{e}_{\mathbb{A}^{1}}[1]
$$

for individual simple coroots. By translation invariance, it suffices to establish the isomorphisms

$$
\left(\iota_{-\alpha_{i}}\right)^{!}\left(\Omega_{q, Q u a n t}^{\text {free }}\right) \simeq \mathrm{e}[-1] .
$$

However, this follows from formula (3.16): indeed, we have

$$
\mathrm{C}\left(U_{q}^{\text {free }}(\check{N})\right) \simeq \underset{i}{\oplus} \mathrm{e}^{-\alpha_{i}}[-1]
$$

To prove (3.20), it remains to show that the !-restriction of $\Omega_{q, \text { Quant }}^{\text {free }}$ to the complement of Conf in Conf vanishes. By induction and factorization, it suffices to show that for $\lambda$ which is not one of the negative simple coroots, we have

$$
\left(\Delta_{\lambda}\right)^{!}\left(\Omega_{q, \text { Quant }}^{\text {free }}\right)=0
$$

Again, by translation invariance, this is equivalent to showing that

$$
\left(\iota_{\lambda}\right)^{!}\left(\Omega_{q, \text { Quant }}^{\text {free }}\right)=0 .
$$

However, this again follows from (3.16) and (3.21).

Combining Proposition 3.5 .2 with (3.17), we obtain:

Corollary 3.5.3. There exists a canonical identification as factorization algebras

$$
\Omega_{q, \text { Quant }}^{\text {cofree }} \simeq j !\left(\stackrel{\circ}{\Omega}_{q}\right)
$$

Combining this with Corollary 3.4.10, we obtain:

Corollary 3.5.4. There exist canonically defined injective maps

$$
\Omega_{q, \text { Quant }}^{\text {sml }} \hookrightarrow \Omega_{q, \text { Quant }}^{\text {DK }} \hookrightarrow j_{*}\left(\stackrel{\circ}{q}_{q}\right)
$$

and canonically defined surjective maps

$$
j_{!}\left(\stackrel{\circ}{\Omega_{q}}\right) \rightarrow \Omega_{q, \text { Quant }}^{\text {Lus }} \rightarrow \Omega_{q, \text { Quant }}^{\text {sm }} .
$$

All of these maps respect the factorization algebra structure. 
3.5.5. Note that on the one hand, we can consider the tautological map

$$
j_{!}\left(\stackrel{\circ}{\Omega}_{q}\right) \rightarrow j_{*}\left(\stackrel{\circ}{\Omega_{q}}\right) .
$$

On the other hand, consider the map

$$
\Omega_{q, \text { Quant }}^{\text {cofree }} \rightarrow \Omega_{q, \text { Quant }}^{\text {free }},
$$

obtained from the map (3.7) by functoriality. We claim:

Lemma 3.5.6. The diagram

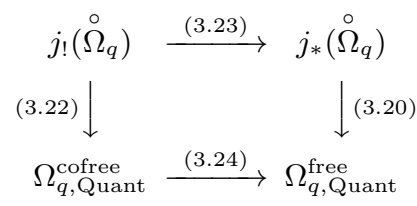

commutes up to a non-zero constant over each $\operatorname{Conf}^{\lambda}$.

Proof. By adjunction, we need to show that the diagram

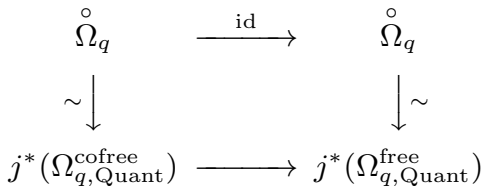

commutes up to a non-zero constant over each $\mathrm{Conf}^{\lambda}$. This is evident since both sides are irreducible.

Remark 3.5.7. One can show that the constant in Lemma 3.5.6 equals 1.

Invoking Corollary 3.4 .10 again, we obtain:

Corollary 3.5.8. There exists an identification

$$
\Omega_{q, \text { Quant }}^{\text {sml }} \simeq j_{! *}\left(\stackrel{\circ}{\Omega_{q}}\right)=: \Omega_{q}^{\text {sml }} .
$$

Finally, we obtain:

Corollary 3.5.9. There exists a string of maps of factorization algebras

$$
j_{!}\left(\stackrel{\circ}{\Omega}_{q}\right) \rightarrow \Omega_{q, \text { Quant }}^{\text {Lus }} \rightarrow \Omega_{q, \text { Quant }}^{\text {sml }} \hookrightarrow \Omega_{q, \text { Quant }}^{\text {DK }} \hookrightarrow j_{*}\left(\stackrel{\circ}{\Omega_{q}}\right),
$$

where all the maps become isomorphisms when restricted to Conf.

Note that the maps in (3.25) are counterparts of the maps in (3.14).

3.5.10. Let us note the following corollary of Corollary 3.3 .12

Corollary 3.5.11. Assume that $q$ is not a root of unity. Then the maps

$$
\Omega_{q, \text { Quant }}^{\text {Lus }} \rightarrow \Omega_{q, \text { Quant }}^{\text {sml }} \hookrightarrow \Omega_{q, \text { Quant }}^{\text {DK }}
$$

are isomorphisms.

\subsection{The first comparison: $\Omega_{q, \mathrm{Kuant}}^{\mathrm{KD}}$ vs abstract $\Omega_{q}^{\mathrm{KD}}$.}

3.6.1. Our current goal is to prove the following result:

Theorem 3.6.2. The embeddings

$$
\Omega_{q, \text { Quant }}^{\mathrm{DK}} \hookrightarrow j_{*}\left(\stackrel{\circ}{\Omega}_{q}\right) \hookleftarrow \Omega_{q}^{\mathrm{DK}}
$$

have equal images.

The rest of this subsection is devoted to the proof of this theorem. 
3.6.3. We will consider the class of Hopf algebras in $\operatorname{Rep}_{q_{\llbracket \hbar \rrbracket}}(\check{T})$ as in Sect. 3.1.4 where the first condition is replaced by the following one:

- Each coweight component $H^{\lambda}$ lies in the heart of $(\mathrm{e} \llbracket \hbar \rrbracket-\mathrm{mod})^{\mathrm{f} . \mathrm{g} .}$ and is $\hbar$-flat.

The equivalence (3.15) extends to a (contravariant) equivalence between the category of such Hopf algebras and that of factorization algebras in $\operatorname{Perv}_{\mathcal{G}_{\llbracket \hbar \hbar}}$ (Conf) that are $\hbar$-flat (see Sect. A.2.5 for what the latter means).

Remark 3.6.4. The flatness condition in the statement of the equivalence is due to the fact that our assignment $H \mapsto \Omega_{H}$ is contravariant. If we worked with the covariant version, we could have omitted the flatness condition on both sides.

3.6.5. Consider the Hopf algebras

$$
U_{q_{\llbracket \hbar \rrbracket}}^{\text {free }}(\check{N}) \rightarrow U_{q_{\llbracket \hbar \rrbracket}}^{\mathrm{DK}}(\check{N})
$$

as well as

$$
U_{q_{((\hbar))}}^{\text {free }}(\check{N}) \rightarrow U_{q_{((\hbar))}}^{\mathrm{DK}}(\check{N}) \hookrightarrow U_{q_{((\hbar))}}^{\text {cofree }}(\check{N}) .
$$

Consider the corresponding factorization algebras

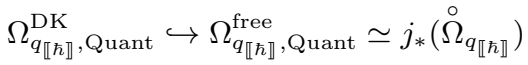

and

$$
j_{!}\left(\stackrel{\circ}{\Omega}_{q_{((\hbar))}}\right) \stackrel{\sim}{\rightarrow} \Omega_{q_{((\hbar))} \text {, Quant }}^{\text {cofree }} \rightarrow \Omega_{q_{((\hbar))}, \text { Quant }}^{\text {DK }} \hookrightarrow \Omega_{q_{((\hbar))} \text {, Quant }}^{\text {free }} \simeq j_{*}\left(\stackrel{\circ}{\Omega}_{q_{((\hbar))}}\right) .
$$

In particular, we obtain an isomorphism

$$
\Omega_{q_{((\hbar))}^{\mathrm{DK}}, \text { Quant }}^{\mathrm{Du}} \simeq \Omega_{q_{((\hbar))}},
$$

(see Sect. 1.7.6, where the latter is defined).

3.6.6. The resulting maps

and

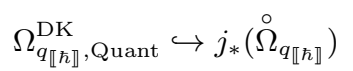

define a map

$$
\Omega_{q_{\llbracket \hbar \rrbracket}^{\mathrm{DK}} \text { Quant }}^{\mathrm{DK}} \hookrightarrow \Omega_{q_{((\hbar))} \text {, Quant }}^{\mathrm{DK}} \simeq \Omega_{q_{((\hbar))}}
$$

We claim that the map (3.26) is an isomorphism. Once this is proved, Theorem 3.6 .2 would follow by reduction $\bmod \hbar$.

3.6.7. We know that it (3.26) is an isomorphism after inverting $\hbar$. Hence, its cokernel is $\hbar$-torsion. Therefore, if this cokernel were non-zero, the map

$$
\Omega_{q_{\llbracket \hbar \rrbracket}^{\mathrm{DK}}, \text { Quant }}^{\mathrm{DK}} / \hbar \rightarrow \Omega_{q_{\llbracket \hbar \rrbracket}^{\mathrm{DK}}}^{\mathrm{D}} / \hbar
$$

would not be injective. However, this would be a contradiction as the latter map is a map

$$
\Omega_{q, \text { Quant }}^{\mathrm{DK}} \rightarrow \Omega_{q}^{\mathrm{DK}},
$$

whose composition with the map

$$
\Omega_{q}^{\mathrm{DK}} \rightarrow j_{*}\left(\stackrel{\circ}{\Omega_{q}}\right)
$$

is the embedding

$$
\Omega_{q, \text { Quant }}^{\mathrm{DK}} \rightarrow j_{*}\left(\stackrel{\circ}{\Omega_{q}}\right) .
$$

$\square[$ Theorem 3.6 .2

3.7. Proof of the properties of $\Omega_{q}^{\mathrm{KD}}$ in characteristic 0 . Having established the isomorphism $\Omega^{\mathrm{DK}} \simeq \Omega_{q, \text { Quant }}^{\mathrm{DK}}$, we can now prove Theorems 2.1.5, 2.2.3, 2.3.4, 2.4.2 and 2.5.2 
3.7.1. By the étale invariance of the construction (see Remark 2.1.3), we can assume that $X=\mathbb{A}^{1}$.

By Lefschetz principle and Riemann-Hilbert, it is enough to do so in the context of the Betti sheaf theory. So we will prove the corresponding assertions for $\Omega_{q, \mathrm{Quant}}^{\mathrm{DK}}$.

3.7.2. First off, the assertion of Theorem 2.1.5 is immediate from

$$
\Omega_{q, \text { Quant }}^{\mathrm{DK}} \stackrel{\text { Theorem }}{\simeq} \Omega_{q}^{\mathrm{DK}} \text { and } \Omega_{q, \text { Quant }}^{\mathrm{sml}} \stackrel{\text { Corollary }}{\simeq} \Omega_{q}^{\mathrm{sml}},
$$

combined with Corollary 3.5 .11

3.7.3. Consider the objects

$$
\left(\Delta_{\lambda}\right)^{!}\left(\Omega_{q, \text { Quant }}^{\text {DK }}\right) \text { and }\left(\Delta_{\lambda}\right)^{*}\left(\Omega_{q, \text { Quant }}^{\text {DK }}\right)
$$

in $\operatorname{Shv}_{\mathcal{G}}\left(\mathbb{A}^{1}\right)$. They are equivariant with respect to the action of $\mathbb{A}^{1}$ on itself by translations. So it is enough to prove the corresponding assertions for the !- and *- fibers, respectively, of these objects at the point

$$
\lambda \cdot 0 \stackrel{\iota_{\lambda}}{\longrightarrow} X^{\lambda}
$$

We will show:

(a) If $q$ satisfies $(*)$ then

$$
\left(\iota_{\lambda}\right)^{!}\left(\Omega_{q, \text { Quant }}^{\mathrm{DK}}\right) \simeq\left\{\begin{array}{l}
\mathrm{e}[-\ell(w)], \quad \lambda=w(\rho)-\rho \\
0, \quad \text { otherwise. }
\end{array}\right.
$$

(b) If $\lambda=w(\rho)-\rho$ with $\ell(w)=2$, then $\left(\iota_{\lambda}\right)^{*}\left(\Omega^{\mathrm{DK}}\right)$ lives in cohomological degrees $\leq-2$, i.e., $H^{-1}\left(\left(\iota_{\lambda}\right)^{*}\left(\Omega^{\mathrm{DK}}\right)\right)=0$.

(b') If $q$ satisfies a sharp inequality $\left(^{*}\right)$ and $\lambda=w(\rho)-\rho$ with $\ell(w) \geq 3$, then $\left(\iota_{\lambda}\right)^{*}\left(\Omega^{\mathrm{DK}}\right)$ lives in cohomological degrees $\leq-2$, i.e., $H^{-1}\left(\left(\iota_{\lambda}\right)^{*}\left(\Omega^{\mathrm{DK}}\right)\right)=0$.

3.7.4. We note that for $\Omega_{q}^{\mathrm{DK}}$ replaced by $\Omega_{q, \mathrm{Quant}}^{\mathrm{DK}}$, the assertion of Theorem 2.2 .3 is equivalent to property (b), the assertions of Theorem 2.3.4 and Theorem 2.4.2 combined are equivalent to property (a) and the assertion of Theorem 2.5 .2 is equivalent to property (b').

The rest of this subsection is devoted to the proof of properties (a), (b) and (b') above.

3.7.5. By (3.16), we have

$$
\left(\iota_{\lambda}\right)^{!}\left(\Omega_{q, \text { Quant }}^{\mathrm{DK}}\right)=\mathrm{C} \cdot\left(U_{q}^{\mathrm{DK}}(\check{N})\right)^{\lambda} .
$$

We now recall the following result of [Geo, Theorem 6.4.1], valid for $q$ satisfying $(*)$ :

$$
\mathrm{C} \cdot\left(U_{q}^{\mathrm{DK}}(\check{N})\right) \simeq \underset{w \in W}{\oplus} \mathrm{e}^{w(\rho)-\rho}[-\ell(w)]
$$

see Remark 2.3.2 This proves (a).

3.7.6. We will now make a digression and show that if $q$ satisfies $\left(^{*}\right)$, then the map (3.13) is an isomorphism.

Let $\mathcal{J}_{q}=\mathcal{J}_{q_{\llbracket \hbar \rrbracket}} / \hbar$ be the kernel of the map $U_{q}^{\text {free }}(\check{N}) \rightarrow U_{q}^{\mathrm{DK}}(\check{N})$. By the spectral sequence,

$$
H^{2}\left(U_{q}^{\mathrm{DK}}(\check{N})\right) \simeq \operatorname{Hom}_{U_{q}^{\text {free }}(\check{N})-\operatorname{bimod}}\left(\mathcal{J}_{q}, \mathrm{e}\right),
$$

where we note that the right-hand in the above formula is the dual space of the (two-sided) quotient $\overline{\mathcal{J}}_{q}$ of $\mathcal{J}_{q}$ by the augmentation ideal of $U_{q}^{\text {free }}(\check{N})$.

Combined with (3.27), we obtain that if $q$ satisfies $(*)$, then $\bar{J}_{q}$ is spanned by elements with coweights

$$
\rho-w(\rho), \quad \ell(w)=2,
$$

i.e.,

$$
\lambda_{i, j}:=-\left(s_{i} \cdot s_{j}(\rho)-\rho\right)=\alpha_{j}+\left(1-\left\langle\alpha_{j}, \check{\alpha}_{i}\right\rangle\right) \cdot \alpha_{i}, \quad i, j \in I
$$

and each such coweight space is one-dimensional. 
Recall the Serre relation elements $S_{q}^{i, j} \in\left(\mathcal{J}_{q}\right)^{\lambda_{i, j}}$, see Sect. 3.2.7 It is easy to see that the image $\bar{S}_{q}^{i, j}$ of $S_{q}^{i, j}$ under

$$
\mathcal{J}_{q} \rightarrow \overline{\mathcal{J}}_{q}
$$

is non-zero. Hence, we obtain that the elements $\bar{S}_{q}^{i, j}$ span $\overline{\mathcal{J}}_{q}$.

Hence, we obtain that if $q$ satisfies $\left(^{*}\right)$, the elements $S_{q}^{i, j}$ generate $\mathcal{J}_{q}$ as a two-sided ideal.

3.7.7. Let us prove (b) and (b'). By (3.17) and (3.16), the fiber $\left(\iota_{\lambda}\right)^{*}\left(\Omega_{q}^{\mathrm{DK}}\right)$ identifies with

$$
\text { C. }\left(U_{q^{-1}}^{\text {Lus }}(\check{N})\right)^{\lambda} \text {. }
$$

We need to show that the latter has no cohomology in degree -1 in coweights of the form $\rho-w(\rho)$.

The vector space $H_{1}\left(U_{q^{-1}}^{\text {Lus }}(\check{N})\right)$ is the quotient of the augmentation ideal of $U_{q^{-1}}^{\text {Lus }}(\check{N})$ by its square, and thus is spanned by the generators of $U_{q^{-1}}^{\text {Lus }}(\check{N})$.

There two kinds of generators: ones corresponding to simple coroots, and ones corresponding to all coroots (the latter are the "divided powers" generators). The generator of the first kind corresponding to a simple coroot $\alpha_{i}$ has coweight $\alpha_{i}$. The generator of the second kind corresponding to a coroot $\alpha$ has coweight $\operatorname{ord}(q(\alpha)) \cdot \alpha$.

Let first $\ell(w)=2$, so $\rho-w(\rho)$ is of the form (3.28). In this case, the assertion follows from the fact that none of the coweights of the form $\alpha_{i}$ or $\operatorname{ord}(q(\alpha)) \cdot \alpha$ have the form (3.28). This proves (b).

Let now $\ell(w) \geq 3$. For the generators of the first kind, we cannot have $\rho-w(\rho)=\alpha_{i}$. For the generators of the second kind, assume that

$$
\rho-w(\rho)=\operatorname{ord}(q(\alpha)) \cdot \alpha
$$

for some coroot $\alpha$.

Evaluating $q_{\mathbb{Z}}(-)+b_{\mathbb{Z}}(-, \rho)$ on both sides, we obtain

$$
\langle\rho, \check{\alpha}\rangle=\operatorname{ord}(q(\alpha)),
$$

which is impossible if $\left(^{*}\right)$ is sharp. This proves (b').

\section{The quantum Frobenius}

In this section we will be assuming that $q$ is torsion-valued, but that it avoids small torsion (see Sect. 1.1.5 for what this means 13 .

We will study the manifestation of Lusztig's quantum Frobenius via the factorization algebras $\Omega_{q}^{\text {Lus }}$ and $\Omega_{q}^{\mathrm{sml}}$.

\subsection{The quantum Frobenius lattice.}

4.1.1. Let $\Lambda^{\sharp} \subset \Lambda$ be the sublattice generated by the elements

$$
\alpha_{i}^{\sharp}=\operatorname{ord}\left(q\left(\alpha_{i}\right)\right) \cdot \alpha_{i} .
$$

Due to the condition that $q \in \operatorname{Quad}(\Lambda, \mathfrak{Z})_{\mathrm{restr}}^{W}$, we have

$$
b(\gamma, \lambda)=0 \text { for all } \gamma \in \Lambda^{\sharp}, \lambda \in \Lambda .
$$

Moreover, the sublattice $\Lambda^{\sharp}$ is $W$-invariant, and hence contains all the elements

$$
\alpha^{\sharp}:=\operatorname{ord}(q(\alpha)) \cdot \alpha \text {. }
$$

Finally, it is known that $\left(\Lambda^{\sharp},\left\{\alpha^{\sharp}\right\}\right)$ is the root system of a simple group of adjoint type, to be denoted $G^{\sharp}$ (over the field of coefficients e), with $\alpha_{i}^{\sharp}$ being the simple roots (see [Lus, Sect. 2.2.4]).

\footnotetext{
${ }^{13}$ The latter condition is needed to ensure a relationship between $U_{q}^{\text {Lus }}(\check{N})$ and $u_{q}(\check{N})$ via the quantum Frobenius, i.e., that 4.30 is a short exact sequence of Hopf algebras.
} 
4.1.2. Let Conf ${ }^{\sharp}$ be the configuration space corresponding to the lattice $\Lambda^{\sharp}$. Let Frob , Conf $_{\text {denote the }}$ tautological closed embedding

$$
\text { Conf }^{\sharp} \rightarrow \text { Conf. }
$$

The functoriality of the construction

$$
q \mapsto \mathcal{G}_{q}^{T} \mapsto \mathcal{G}_{q}^{\mathrm{Conf}}
$$

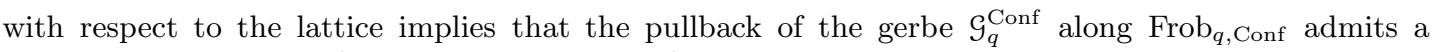
canonical trivialization (as a factorization gerbe).

4.1.3. Recall that add denotes the addition operation on divisors

$$
\text { add : Conf } \times \text { Conf } \rightarrow \text { Conf; }
$$

it makes Conf into a commutative semi-group.

The operation

$$
\mathcal{F}_{1}, \mathcal{F}_{2} \in \operatorname{Shv}(\operatorname{Conf}) \mapsto \mathcal{F}_{1} \star \mathcal{F}_{2}:=\operatorname{add}_{!}\left(\mathcal{F}_{1} \otimes \mathcal{F}_{2}\right) \in \operatorname{Shv}(\operatorname{Conf})
$$

makes Shv(Conf) into a (symmetric) monoidal category. We will refer to this (symmetric) monoidal structure as convolution; this is in order to distinguish it from the pointwise $\stackrel{!}{\otimes}$ symmetric monoidal structure.

In practice we will apply this to the lattice $\Lambda^{\sharp}$, rather than $\Lambda$.

4.1.4. Let act denote the addition map

$$
\text { act : Conf } \sharp \text { Conf } \rightarrow \text { Conf; }
$$

it makes Conf into a Conf ${ }^{\sharp}$-module.

Note that due to factorization and the trivialization of $\left.\mathcal{G}_{q}^{\text {Conf }}\right|_{\text {Conf }}$, we have a canonical isomorphism

$$
\left.\left.\operatorname{act}^{*}\left(\mathcal{G}_{q}^{\text {Conf }}\right)\right|_{(\text {Conf } \sharp \times \text { Conf })_{\text {disj }}} \simeq \operatorname{pr}^{*}\left(\mathcal{G}_{q}^{\text {Conf }}\right)\right|_{(\text {Conf } \sharp \times \text { Conf })_{\text {disj }}},
$$

where pr is the projection on the Conf factor.

However, due to (4.1), the identification (4.2) extends (automatically, uniquely) to an identification

$$
\operatorname{act}^{*}\left(\mathcal{G}_{q}^{\text {Conf }}\right) \simeq \operatorname{pr}^{*}\left(\mathcal{G}_{q}^{\text {Conf }}\right)
$$

over all of Conf $\sharp \times$ Conf, see (1.11). This identification is (automatically) compatible with the semigroup structure on $\mathrm{Conf}^{\sharp}$.

Thus, we obtain that $\mathcal{G}_{q}^{\text {Conf }}$ is equivariant with respect to the action of Conf ${ }^{\sharp}$ on Conf, in a way compatible with factorization.

We obtain that the operation

$$
\mathcal{F}^{\sharp} \in \operatorname{Shv}\left(\operatorname{Conf}^{\sharp}\right), \mathcal{F} \in \operatorname{Shv}_{\mathcal{G}_{q}^{\text {Conf }}}(\operatorname{Conf}) \mapsto \mathcal{F}^{\sharp} \star \mathcal{F}:=\operatorname{act}_{!}\left(\mathcal{F}^{\sharp} \otimes \mathcal{F}\right) \in \operatorname{Shv}_{\mathcal{G}_{q}^{\text {Conf }}}(\operatorname{Conf})
$$

defines an action of the monoidal category $\operatorname{Shv}\left(\operatorname{Conf}^{\sharp}\right)$ on $\operatorname{Shv}_{\mathcal{G}_{q}^{\text {Conf }}}($ Conf $)$. We will refer to this action also as convolution.

\subsection{Convolution factorization algebras.}


4.2.1. Let $\mathcal{A}$ be a factorization algebra in $\mathrm{Shv}(\mathrm{Conf})$. The structure of (commutative) semi-group on Conf allows us to talk about an associative (resp., commutative) algebra structure on $\mathcal{A}$ :

By definition, such a structure consists of a map

$$
\mathcal{A} \star \mathcal{A} \rightarrow \mathcal{A},
$$

compatible with factorization and the associativity (resp., and the commutativity) law on Conf. In particular, such an $\mathcal{A}$ is an associative (resp., commutative) algebra object in $\operatorname{Shv}($ Conf) with respect to convolution.

Remark 4.2.2. Note that if $\mathcal{A}_{1}$ and $\mathcal{A}_{2}$ are factorization algebras on Conf, then their convolution $\mathcal{A}_{1} \star \mathcal{A}_{2}$ will not be a factorization algebra. Rather,

$$
\mathcal{A}_{1} \oplus\left(\mathcal{A}_{1} \star \mathcal{A}_{2}\right) \oplus \mathcal{A}_{2}
$$

will be a factorization algebra.

This can be rephrased as follows. Let $\mathrm{Conf}^{+}$be the disjoint union Conf $\sqcup$ pt. Then the structure of commutative semi-group on Conf extends to a structure of commutative monoid on Conf ${ }^{+}$with pt being the unit. This extends the structure of non-unital symmetric monoidal category on Shv(Conf) to a unital one on $\operatorname{Shv}\left(\mathrm{Conf}^{+}\right)$.

Given a factorization algebra $\mathcal{A}$ on Conf, we extend it to $\mathcal{A}^{+} \in \operatorname{Shv}\left(\operatorname{Conf}^{+}\right)$by setting $\left.\mathcal{A}^{+}\right|_{\mathrm{pt}}=\mathrm{e}$. With these conventions, for a pair of factorization algebras $\mathcal{A}_{1}$ and $\mathcal{A}_{2}$ on Conf,

$$
\mathcal{A}_{1}^{+} \star \mathcal{A}_{2}^{+} \in \operatorname{Shv}\left(\mathrm{Conf}^{+}\right)
$$

is a factorization algebra, and its restriction back to Conf equals (4.5).

This procedure mimics the following: let $\mathcal{C}$ be a unital symmetric monoidal category. For an algebra $\mathcal{A} \in \mathcal{C}$, let $\mathcal{A}^{+}:=\mathcal{A} \oplus \mathbf{1}_{\mathcal{C}}$ be the corresponding unital augmented algebra. Then we have

$$
\mathcal{A}_{1}^{+} \otimes \mathcal{A}_{2}^{+} \simeq\left(\mathcal{A}_{1} \oplus\left(\mathcal{A}_{1} \otimes \mathcal{A}_{2}\right) \oplus \mathcal{A}_{2}\right) \oplus \mathbf{1}_{\mathcal{C}} .
$$

4.2.3. Note that the map (4.4) by adjunction corresponds to a map

$$
\mathcal{A} \otimes \mathcal{A} \rightarrow \operatorname{add}^{!}(\mathcal{A}) .
$$

The compatibility of the algebra structure and factorization implies, in particular, that the restriction of the map (4.6) to $(\text { Conf } \times \text { Conf })_{\text {disj }}$ coincides with the factorization isomorphism

$$
\left.\left.\mathcal{A} \otimes \mathcal{A}\right|_{(\operatorname{Conf} \times \operatorname{Conf})_{\mathrm{disj}}} \simeq \operatorname{add}^{!}(\mathcal{A})\right|_{(\operatorname{Conf} \times \operatorname{Conf})_{\mathrm{disj}}} .
$$

4.2.4. Set

$$
\mathcal{A}_{X}:=\bigoplus_{\gamma}\left(\Delta_{\gamma}\right)^{!}(\mathcal{A}) \in \operatorname{Shv}(X)^{\Lambda} .
$$

By !-restricting (4.4) to the main diagonals, we obtain that a structure on $\mathcal{A}$ of associative (resp., commutative) algebra with respect to $\star$ induces a structure of associative (resp., commutative) algebra on $\mathcal{A}_{X}$, with respect to the symmetric monoidal structure on $\operatorname{Shv}(X)^{\Lambda}$, induced by the $\stackrel{!}{\otimes}$ (symmetric) monoidal structure on $\operatorname{Shv}(X)$ and the addition operation on $\Lambda$.

Remark 4.2.5. The algebra $\mathcal{A}_{X}$ is non-unital. The corresponding unital algebra

$$
\mathcal{A}_{X}^{+}:=\mathcal{A}_{X} \oplus \omega_{X}
$$

can be obtained from $\mathcal{A}^{+}$(see Remark 4.2.2) also by pullback, where the corresponding map

$$
\Delta_{0}: X \rightarrow \text { Conf }^{+}
$$

is

$$
X \rightarrow \text { pt } \hookrightarrow \text { Conf }^{+} .
$$


4.2.6. For $x \in X$, let $\operatorname{Conf}_{x} \subset$ Conf be the closed subset equal to the union of the images of the maps

$$
\text { Conf } \simeq \text { pt } \times \text { Conf } \stackrel{{ }^{-\alpha_{i}} \times \text { id }}{\longrightarrow} \text { Conf } \times \text { Conf } \stackrel{\text { add }}{\rightarrow} \text { Conf }
$$

I.e., Conf ${ }_{x}$ is the closed subset consisting of those divisors that have a non-trivial contribution at $x$.

Let $\mathcal{F}_{1}$ and $\mathcal{F}_{2}$ be a pair of perverse sheaves on Conf that do not have sub-objects supported at subsets of the form $\operatorname{Conf}_{x}$ (for the same $x$ appearing on both sides). In this case the map

$$
\left(j_{\text {disj }}\right) ! \circ\left(j_{\text {disj }}\right)^{*}\left(\mathcal{F}_{1} \otimes \mathcal{F}_{2}\right) \rightarrow \mathcal{F}_{1} \otimes \mathcal{F}_{2}
$$

is surjective as a map of perverse sheaves on Conf $\times$ Conf. (Here $j_{\text {disj }}$ denotes the open embedding $(\text { Conf } \times \text { Conf })_{\text {disj }} \hookrightarrow$ Conf $\times$ Conf.)

Let $\mathcal{A}$ be a factorization algebra in $\operatorname{Perv}\left(\right.$ Conf), such that $\mathcal{A}_{X}$ does not have quotient objects supported at closed points of $X$. This implies that $\mathcal{A}$ does not have quotient objects supported on subsets of the form $\operatorname{Conf}_{x}$.

We obtain that in this case, if the factorization isomorphism (4.7), viewed as a map

$$
\left.\left.\mathcal{A} \otimes \mathcal{A}\right|_{(\operatorname{Conf} \times \text { Conf })_{\mathrm{disj}}} \rightarrow \operatorname{add}(\mathcal{A})\right|_{(\operatorname{Conf} \times \operatorname{Conf})_{\mathrm{disj}}},
$$

extends to all of Conf $\times$ Conf, then it does so uniquely.

Thus, we obtain that under the above condition, being an associative algebra within factorization algebras is a condition and not an additional structure. Furthermore, in this case, the associative algebra structure is automatically commutative.

4.2.7. Given a factorization algebra $\mathcal{A}^{\sharp} \in \operatorname{Shv}\left(\operatorname{Conf}^{\sharp}\right)$, equipped with an associative algebra structure, and a factorization algebra $\mathcal{A} \in \operatorname{Shv}_{\mathcal{G}_{q}^{\text {Conf }}}\left(\right.$ Conf), one can talk about an action of $\mathcal{A}^{\sharp}$ on $\mathcal{A}$ :

By an action we will mean the datum of a map of factorization algebras

$$
\left(\operatorname{Frob}_{q, \text { Conf }}\right) !\left(\mathcal{A}^{\sharp}\right) \rightarrow \mathcal{A}
$$

and a map

$$
\mathcal{A}^{\sharp} \star \mathcal{A} \rightarrow \mathcal{A},
$$

that make the following diagram commute:

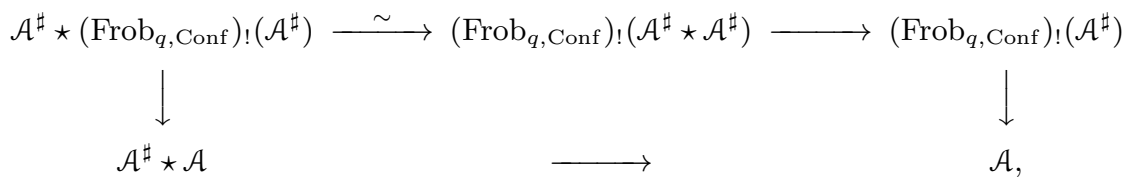

in a way compatible with factorization and the associativity law.

Remark 4.2.8. The fact that we have to specify the map (4.9) in addition to (4.10) is the reflection of the non-unital nature of our symmetric monoidal categories, see Remark 4.2.2.

These two pieces of data can be combined into one:

$$
\left(\mathcal{A}^{\sharp}\right)^{+} \star \mathcal{A}^{+} \rightarrow \mathcal{A}^{+},
$$

subject to the associativity law.

4.2.9. The map (4.10) can be expressed by adjunction as a map

$$
\left(\operatorname{Frob}_{q, \mathrm{Conf}}\right) !\left(\mathcal{A}^{\sharp}\right) \otimes \mathcal{A} \rightarrow \operatorname{add}^{!}(\mathcal{A})
$$

on Conf $\times$ Conf.

Again, the compatibility with factorization implies that the restriction of the restriction of the map (4.12) to $(\text { Conf } \times \text { Conf })_{\text {disj }}$ indentifies with

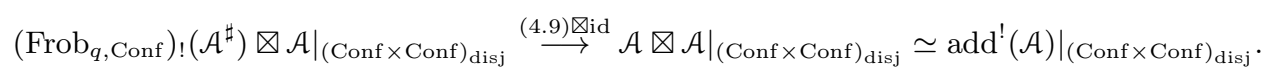


4.2.10. Let us be given an action of $\mathcal{A}^{\sharp}$ on $\mathcal{A}$, and consider $\mathcal{A}_{X}^{\sharp}$ and $\mathcal{A}_{X}$ (defined as in (4.8)).

We obtain a map

$$
\mathcal{A}_{X}^{\sharp} \rightarrow \mathcal{A}_{X}
$$

and an action of $\mathcal{A}_{X}^{\sharp}$, viewed as an algebra object in the (symmetric) monoidal category $\operatorname{Shv}(X)^{\Lambda^{\sharp}}$, on $\mathcal{A}_{X}$ (that are compatible via an analog of the diagram (4.11)), where we view

$$
\operatorname{Shv}_{\mathcal{G}_{q}^{\lambda}}(X)^{\Lambda}:=\bigoplus_{\lambda} \operatorname{Shv}_{\mathcal{G}_{q}^{\lambda}}(X)
$$

as an $\operatorname{Shv}(X)^{\Lambda^{\sharp}}$-module category.

Remark 4.2.11. This data can be equivalently expressed as an action of $\left(\mathcal{A}_{X}^{\sharp}\right)^{+}$on $\mathcal{A}_{X}^{+}$.

4.2.12. Finally, let assume that both $\mathcal{A}^{\sharp}$ and $\mathcal{A}$ are perverse and such that $\mathcal{A}_{X}^{\sharp}$ and $\mathcal{A}_{X}$ do not have quotient objects supported at closed points of $x$.

Then as in Sect. 4.2.6 we obtain that given a datum of (4.9), an action of $\mathcal{A}^{\sharp}$ on $\mathcal{A}$ is not an additional structure, but rather a condition. Namely, this condition says that the map (4.13) extends to all of Conf $\times$ Conf (if it does, this extension is unique).

4.3. The classical factorization algebra $\Omega^{\sharp, c l}$. In this subsection we will construct a certain factorization algebra, denoted $\Omega^{\text {cl }}$ in Perv(Conf). In practice, we will apply this construction to the lattice $\Lambda^{\sharp}$ rather than $\Lambda$; in this case, the resulting factorization algebra will be denoted by $\Omega^{\sharp \text {,cl }}$.

4.3.1. Recall (following, e.g., Ga2, Sect. 2.3]) that to a commutative algebra object $A$ in the (nonunital) symmetric monoidal category $\operatorname{Vect}^{\Lambda^{\text {neg }}-0}$, one canonically attaches a factorization algebra Fact $(A)$ on Conf, equipped with a structure of commutative algebra in the sense of Sect. 4.2 .1

We have:

$$
\operatorname{Fact}(A)_{X} \simeq \omega_{X} \otimes A,
$$

as commutative algebras in $\operatorname{Shv}(X)^{\Lambda}$, see Sect.4.2.4 for the notation.

In fact, the assignment

$$
A \mapsto \operatorname{Fact}(A)
$$

is the composition of the pullback functor

$$
\operatorname{ComAlg}\left(\operatorname{Vect}{ }^{\Lambda^{\text {neg }}-0}\right) \rightarrow \operatorname{ComAlg}\left(\operatorname{Shv}(X)^{\Lambda^{\text {neg }}-0}\right), \quad A \mapsto \omega_{X} \otimes A
$$

followed by an equivalence of categories

$$
\operatorname{ComAlg}\left(\operatorname{Shv}(X)^{\Lambda^{\text {neg }}-0}\right) \rightarrow \operatorname{ComAlg}(\operatorname{FactAlg}(\operatorname{Shv}(\operatorname{Conf}))),
$$

where the functor inverse to (4.17) is given by $\mathcal{A} \mapsto \mathcal{A}_{X}$.

Remark 4.3.2. When the sheaf theory we are working with is that of sheaves in the classical topology, the functor (4.16) is a special case of the functor (3.15) for $q=1$.

Namely, the two procedures are related via the Koszul duality equivalence between the category of co-commutative Hopf algebras as in Sect. 3.1.4 and $\operatorname{ComAlg}\left(\left(\operatorname{Vect}{ }^{\Lambda^{\text {neg }}-0}\right)^{\text {loc.fin.dim. }}\right)$ :

For a Hopf algebra $H$, the associative algebra

$$
\mathrm{C} \cdot(H)
$$

has a natural structure of $\mathbb{E}_{2}$-algebra, and if $H$ was cocommutative, this $\mathbb{E}_{2}$-algebra structure naturally upgrades to an $\mathbb{E}_{\infty}$-structure. 
4.3.3. We will apply the above construction to (the augmentation ideal of)

$$
A:=\mathrm{C} \cdot(\check{\mathfrak{n}}) \text {. }
$$

Denote the resulting factorization algebra by $\Omega^{\mathrm{cl}}$.

Remark 4.3.4. As was mentioned above, in practice we will apply this construction to $\Lambda^{\sharp}$ rather than $\Lambda$, and consider the factorization algebra

$$
\Omega^{\sharp, c l}:=\operatorname{Fact}\left(\mathrm{C}^{\cdot}\left(\mathfrak{n}^{\sharp}\right)\right) \in \operatorname{Shv}\left(\operatorname{Conf}^{\sharp}\right),
$$

where $\mathfrak{n}^{\sharp}$ is the maximal unipotent in the group $G^{\sharp}$ from Sect. 4.1.1.

4.3.5. Since $(A)^{-\alpha_{i}}=\mathrm{e}[-1]$, from (4.15) we obtain that

$$
\left.\Omega^{\mathrm{cl}}\right|_{\mathrm{Conf}-\alpha_{i}}=\mathrm{e}_{X}[1] .
$$

Hence, we obtain a canonical identification of factorization algebras over Conf:

$$
j^{*}\left(\Omega^{\mathrm{cl}}\right) \simeq \stackrel{\circ}{\Omega} .
$$

Consider the resulting map

$$
\Omega^{\mathrm{cl}} \rightarrow j_{*}(\stackrel{\circ}{\Omega}) .
$$

The following is established in BG2, Sect. 3 and Lemma 4.8]:

Theorem 4.3.6. The factorization algebra $\Omega^{\mathrm{cl}}$ is perverse, and the map (4.18) is injective.

In fact, the proof in Sect. 3.7 applies to $\Omega^{\mathrm{cl}}$, giving rise to the following explicit inductive description of $\Omega^{\mathrm{cl}}$ as a sub-object of $j_{*}(\stackrel{\circ}{\Omega})$ :

Theorem 4.3.7. For $\lambda \in \Lambda^{\text {neg }}$, the maps

$$
\left.\left(\jmath_{\lambda}\right) ! * \circ\left(\jmath_{\lambda}\right)^{*}\left(\Omega^{\mathrm{cl}}\right) \rightarrow \Omega^{\mathrm{cl}}\right|_{\mathrm{Conf} \lambda} \rightarrow H^{0}\left(\left(\jmath_{\lambda}\right)_{*} \circ\left(\jmath_{\lambda}\right)^{*}\left(\Omega^{\mathrm{cl}}\right)\right) \rightarrow\left(\jmath_{\lambda}\right)_{*} \circ\left(\jmath_{\lambda}\right)^{*}\left(\Omega^{\mathrm{cl}}\right)
$$

have the following proprties:

(a) For $\lambda=w(\rho)-\rho$ with $\ell(w) \geq 2$, the map $\left.\left(\jmath_{\lambda}\right) !_{*} \circ\left(\jmath_{\lambda}\right)^{*}\left(\Omega^{\mathrm{cl}}\right) \rightarrow \Omega^{\mathrm{cl}}\right|_{\mathrm{Conf}^{\lambda}}$ is an isomorphism.

(b) For $\lambda=w(\rho)-\rho$ with $\ell(w) \geq 2$, the cone of the map

$$
\left.\Omega^{\mathrm{cl}}\right|_{\text {Conf } \lambda} \rightarrow\left(\jmath_{\lambda}\right)_{*} \circ\left(\jmath_{\lambda}\right)^{*}\left(\Omega^{\mathrm{cl}}\right)
$$

identifies with $\left(\Delta_{\lambda}\right)_{*}\left(\mathrm{e}_{X}[-\ell(w)+1]\right)$. In particular:

(i) If $\ell(w)=2$, the object $\left(\jmath_{\lambda}\right)_{*} \circ\left(\jmath_{\lambda}\right)^{*}\left(\Omega^{\mathrm{cl}}\right)$ is perverse;

(ii) If $\ell(w) \geq 3$, the map

$$
\left.\Omega^{\mathrm{cl}}\right|_{\operatorname{Conf}^{\lambda}} \rightarrow H^{0}\left(\left(\jmath_{\lambda}\right)_{*} \circ\left(\jmath_{\lambda}\right)^{*}\left(\Omega^{\mathrm{cl}}\right)\right)
$$

is an isomorphism.

(c) For $\lambda$ not of the form $w(\rho)-\rho$, the maps

$$
\left.\Omega^{\mathrm{cl}}\right|_{\mathrm{Conf} \lambda} \rightarrow H^{0}\left(\left(\jmath_{\lambda}\right)_{*} \circ\left(\jmath_{\lambda}\right)^{*}\left(\Omega^{\mathrm{cl}}\right)\right) \rightarrow\left(\jmath_{\lambda}\right)_{*} \circ\left(\jmath_{\lambda}\right)^{*}\left(\Omega^{\mathrm{cl}}\right)
$$

are isomorphisms.

Corollary 4.3.8. The map

$$
\left.\Omega^{\mathrm{cl}}\right|_{\text {Conf } \lambda} \rightarrow H^{0}\left(\left(\jmath_{\lambda}\right)_{*} \circ\left(\jmath_{\lambda}\right)^{*}\left(\Omega^{\mathrm{cl}}\right)\right)
$$

is an isomorphism for all $\lambda$ not of the form $w(\rho)-\rho$ with $\ell(w)=2$.

Remark 4.3.9. Note that points (a) and (b) of Theorem 4.3.7 for $\lambda=w(\rho)-\rho$ with $\ell(w) \geq 3$ mean that in this case, the maps

$$
\left.\left(\jmath_{\lambda}\right)_{! *} \circ\left(\jmath_{\lambda}\right)^{*}\left(\Omega^{\mathrm{cl}}\right) \rightarrow \Omega^{\mathrm{cl}}\right|_{\operatorname{Conf}^{\lambda}} \rightarrow H^{0}\left(\left(\jmath_{\lambda}\right)_{*} \circ\left(\jmath_{\lambda}\right)^{*}\left(\Omega^{\mathrm{cl}}\right)\right)
$$

are both isomorphisms. 
Remark 4.3.10. One can use the proof of Theorem 3.6.2 to show that the factorization algebra $\Omega^{\mathrm{cl}}$ is in fact a special case of $\Omega_{q}^{\mathrm{DK}}$ (constructed by deforming the parameter in Sect. 1.7) for $q=1$.

\subsection{Relationship of $\Omega_{q}^{\mathrm{Lus}}$ with $\Omega_{q}^{\mathrm{sml}}$ via $\Omega^{\mathrm{cl}}$.}

4.4.1. We will now state a key theorem that expresses the relationship of $\Omega_{q}^{\mathrm{Lus}}$ and $\Omega_{q}^{\mathrm{sml}}$ via $\Omega_{q}^{\sharp, c l}$. (We emphasize that for the validity of this theorem, the assumption that $q$ avoid small torsion is important.)

Theorem 4.4.2.

(a) For every vertex of the Dynkin diagram, we have

$$
H^{0}\left(\left(\Delta_{-\alpha_{i}^{\sharp}}\right)^{!}\left(\Omega_{q}^{\text {Lus }}\right)\right) \simeq \mathrm{e}_{X}[1] .
$$

In particular, we have a have an isomorphism of factorization algebras in $\operatorname{Perv}\left(\mathrm{Conf}^{\sharp}\right)$ :

$$
\left(j^{\sharp}\right)^{*}\left(H^{0}\left(\left(\text { Frob }_{q, \text { Conf }}\right)^{!}\left(\Omega_{q}^{\text {Lus }}\right)\right)\right) \simeq \stackrel{\circ}{\sharp} .
$$

(b) The map

$$
H^{0}\left(\left(\text { Frob }_{q, \text { Conf }}\right)^{!}\left(\Omega_{q}^{\text {Lus }}\right)\right) \rightarrow\left(j^{\sharp}\right)_{*}\left(\stackrel{\circ}{ }^{\sharp}\right)
$$

is injective.

(c) The image of the map (4.20) equals that of $\Omega^{\sharp, c l}$. In particular, we have an isomorphism of factorization algebras

$$
\Omega^{\sharp, \mathrm{cl}} \simeq H^{0}\left(\left(\operatorname{Frob}_{q, \mathrm{Conf}}\right)^{!}\left(\Omega_{q}^{\mathrm{Lus}}\right)\right)
$$

(d) The map

$$
\left(\operatorname{Frob}_{q}, \mathrm{Conf}\right) !\left(\Omega^{\sharp, \mathrm{cl}}\right) \rightarrow \Omega_{q}^{\mathrm{Lus}},
$$

resulting from (4.21) extends to an action of $\Omega^{\sharp, c l}$ on $\Omega_{q}^{\mathrm{Lus}}$.

(e) The projection $\Omega_{q}^{\mathrm{Lus}} \rightarrow \Omega_{q}^{\mathrm{sml}}$ is compatible with $\Omega^{\sharp, \mathrm{cl}}$-actions, where the action on $\Omega_{q}^{\mathrm{sml}}$ is the trivial one.

(f) The resulting map

$$
\left(\operatorname{Cone}\left(\left(\Omega^{\sharp, c l}\right)_{X} \rightarrow\left(\Omega_{q}^{\mathrm{Lus}}\right)_{X}\right)\right) \underset{\left(\Omega^{\sharp}, \mathrm{cl}\right)_{X}}{\otimes} \omega_{X} \rightarrow\left(\Omega_{q}^{\mathrm{sml}}\right)_{X}
$$

is an isomorphism.

Remark 4.4.3. The reason that in point (f) we have the somewhat bizarre looking

$$
\left(\operatorname{Cone}\left(\left(\Omega^{\sharp, c l}\right)_{X} \rightarrow\left(\Omega_{q}^{\mathrm{Lus}}\right)_{X}\right)\right)_{\left(\Omega^{\sharp}, \mathrm{cl}\right)_{X}}^{\otimes} \omega_{X}
$$

instead of just

$$
\left(\left(\Omega^{\sharp, \text { Lus }}\right)_{X}\right)_{\left(\Omega^{\sharp, c l}\right)_{X}}^{\otimes} \omega_{X}
$$

is that we are working in the non-unital setting. E.g., our operation of coinvariants with the cone is designed so that it would send $\Omega^{\sharp \text {,cl }}$ itself to 0 .

We could equivalently rewrite (4.22) as an isomorphism

$$
\left(\Omega_{q}^{\mathrm{Lus}}\right)_{X}^{+} \underset{\left(\Omega_{X}^{\sharp, c l}\right)^{+}}{\otimes} \omega_{X} \simeq\left(\Omega_{q}^{\mathrm{sml}}\right)_{X}^{+} .
$$


Remark 4.4.4. Given an action of $\mathcal{A}^{\sharp} \in \operatorname{Shv}\left(\mathrm{Conf}^{\sharp}\right)$ on $\mathcal{A} \in \operatorname{Shv}_{\mathcal{G}_{q}^{\text {Conf }}}($ Conf $)$, we can form the object

$$
\operatorname{Bar}\left(\mathcal{A}^{\sharp}, \mathcal{A}\right):=\operatorname{Bar}\left(\left(\mathcal{A}^{\sharp}\right)^{+}, \mathcal{A}^{+}\right) \in \operatorname{Shv}_{\mathcal{G}_{q} \text { Conf }}(\text { Conf }),
$$

which also carries a structure of factorization algebra.

We have

$$
\operatorname{Bar}\left(\mathcal{A}^{\sharp}, \mathcal{A}\right)_{X} \simeq \operatorname{Bar}\left(\mathcal{A}_{X}^{\sharp}, \mathcal{A}_{X}\right):=\operatorname{Bar}\left(\left(\mathcal{A}_{X}^{\sharp}\right)^{+}, \mathcal{A}_{X}^{+}\right) \simeq\left(\operatorname{Cone}\left(\mathcal{A}_{X}^{\sharp} \rightarrow \mathcal{A}_{X}\right)\right) \underset{\mathcal{A}_{X}^{\sharp}}{\otimes} \omega_{X} .
$$

So, by factorization, Theorem 4.4 .2 is equivalent to the statement that the map

$$
\Omega_{q}^{\mathrm{Lus}} \rightarrow \Omega_{q}^{\mathrm{sml}}
$$

induces an isomorphism

$$
\operatorname{Bar}\left(\Omega^{\sharp, \mathrm{cl}}, \Omega_{q}^{\mathrm{Lus}}\right) \simeq \Omega_{q}^{\mathrm{sml}} .
$$

4.4.5. Theorem 4.4.2 will be proved in the case of a ground field of characteristic 0 in the rest of this section. The general case will be treated in Sect. 6.6.4.

4.4.6. In the case of when the ground field has characteristic 0, as in Sect. 3.7, we reduce the assertion to the case when $X=\mathbb{A}^{1}$ and the sheaf theory being that of constructible sheaves in the classical topology. Consider the corresponding factorization algebra $\Omega_{q, \text { Quant }}^{\mathrm{Lus}}$, which we already know is isomorphic to $\Omega_{q}^{\mathrm{Lus}}$.

4.4.7. In Sect. 4.6 we will show that there exists a canonically defined action of $\Omega^{\sharp, c l}$ on $\Omega_{q, \text { Quant }}^{\text {Lus }}$ such that the map

$$
\Omega_{q, \text { Quant }}^{\text {Lus }} \rightarrow \Omega_{q, \text { Quant }}^{\text {sml }}
$$

is compatible with the trivial $\Omega^{\sharp, c l}$-action on $\Omega_{q, \text { Quant }}^{\text {sml }}$ and induces an identification

$$
\left(\left(\text { Cone }\left(\Omega^{\sharp, c l}\right)_{X} \rightarrow\left(\Omega_{q, \text { Quant }}^{\text {Lus }}\right)_{X}\right)\right) \underset{\left(\Omega^{\sharp, c l}\right)_{X}}{\otimes} \omega_{X} \rightarrow\left(\Omega_{q, \text { Quant }}^{\text {sml }}\right)_{X}
$$

Let us show how the existence of this action and the isomorphism (4.25) implies the assertion of Theorem 4.4.2

Remark 4.4.8. We emphasize that for the above structure to exist on $\Omega_{q, \text { Quant }}^{\text {Lus }} \simeq \Omega_{q}^{\text {Lus }}$, the assumption that $q$ should avoid small torsion is important. This is due to the fact that the construction of this structure uses (and is essentially equivalent to) the quantum Frobenius for $U_{q}^{\mathrm{Lus}}(\check{N})$.

4.4.9. We only need to show that the map

$$
\left(\operatorname{Frob}_{q, \text { Conf }}\right) !\left(\Omega^{\sharp, c l}\right) \rightarrow \Omega_{q, \text { Quant }}^{\text {Lus }}
$$

induces an isomorphism

$$
\Omega^{\sharp, c l} \rightarrow H^{0}\left(\left(\operatorname{Frob}_{q, \text { Conf }}\right)^{!}\left(\Omega_{q, \text { Quant }}^{\text {Lus }}\right)\right) .
$$

By induction and factorization, this is equivalent to showing that for any $\gamma \in \Lambda^{\sharp \text {,neg }}-0$,

$$
\left(\Delta_{\gamma}\right)^{!}\left(\operatorname{Cone}\left(\left(\operatorname{Frob}_{q, \text { Conf }}\right) !\left(\Omega^{\sharp, \text { cl }}\right) \rightarrow \Omega_{q, \text { Quant }}^{\text {Lus }}\right)\right)
$$

lives in cohomological degrees $\geq 1$.

By translation invariance, it suffices to show that

$$
\left(\iota_{\gamma}\right) !\left(\operatorname{Cone}\left(\left(\operatorname{Frob}_{q, \text { Conf }}\right) !\left(\Omega^{\sharp, \mathrm{cl}}\right) \rightarrow \Omega_{q, \text { Quant }}^{\text {Lus }}\right)\right)
$$

lives in cohomological degrees $\geq 2$. 
4.4.10. Let us denote by $M$ the $\left(\Lambda^{\text {neg }}-0\right)$-graded vector space

$$
\underset{\lambda}{\oplus}\left(\iota_{\lambda}\right)^{!}\left(\operatorname{Cone}\left(\left(\text { Frob }_{q, \text { Conf }}\right) !\left(\Omega^{\sharp, c l}\right) \rightarrow \Omega_{q, \text { Quant }}^{\text {Lus }}\right)\right) .
$$

It is acted on by the $\left(\Lambda^{\sharp \text {,neg }}-0\right)$-graded algebra

$$
\underset{\gamma}{\oplus}\left(\iota_{\gamma}\right)^{!}\left(\Omega^{\sharp, c l}\right) \simeq \mathrm{C}^{\cdot}\left(\mathfrak{n}^{\sharp}\right) .
$$

Isomorphism (4.25) implies that

$$
M \underset{\mathrm{C} \cdot(\mathfrak{n} \sharp)}{\otimes} \mathrm{e} \simeq \underset{\lambda}{\oplus}\left(\iota_{\lambda}\right)^{!}\left(\Omega_{q, \text { Quant }}^{\text {sml }}\right)=: M^{\prime} .
$$
$\Lambda^{\sharp}$.

We need to show that $H^{0}(M)$ and $H^{1}(M)$ do not have non-zero components of degrees that lie in

4.4.11. The datum of action of $\mathrm{C}^{\cdot}\left(\mathfrak{n}^{\sharp}\right)$ on $M$ and the isomorphism (4.26) is equivalent to that of action of the algebraic group $N^{\sharp}$ on $M^{\prime}$ and an isomorphism

$$
\mathrm{C} \cdot\left(N^{\sharp}, M^{\prime}\right) \simeq M .
$$

We know that $M^{\prime}$ is concentrated in cohomological degrees $\geq 1$, and

$$
H^{1}\left(M^{\prime}\right) \simeq \bigoplus_{i} \mathrm{e}^{-\alpha_{i}}
$$

Hence, by the spectral sequence,

$$
H^{0}(M)=0
$$

and

$$
H^{1}(M) \simeq H^{1}\left(M^{\prime}\right) \simeq \underset{i}{\oplus_{i}} \mathrm{e}^{-\alpha_{i}}
$$

This proves the desired assertion since none of the elements $-\alpha_{i}$ lie in $\Lambda^{\sharp}$, due to the non-degeneracy assumption on $q$.

\subsection{The quantum Frobenius map for quantum groups.}

4.5.1. Let $T^{\sharp}$ denote the torus, whose lattice of characters is $\Lambda^{\sharp}$; we can identify $T^{\sharp}$ with the Cartan subgroup of $G^{\sharp}$, see Sect. 4.1.1.

The embedding $\Lambda^{\sharp} \rightarrow \Lambda$ gives rise to a map

$$
\operatorname{Rep}\left(T^{\sharp}\right) \rightarrow Z_{\mathbb{E}_{3}}\left(\operatorname{Rep}_{q}(\check{T})\right) .
$$

At the level of abelian categories (which is all we need for the moment), this means that we have a braided monoidal functor

$$
\operatorname{Frob}_{q, T}^{*}:\left(\operatorname{Rep}\left(T^{\sharp}\right)\right)^{\odot} \rightarrow\left(\operatorname{Rep}_{q}(\check{T})\right)^{\odot},
$$

such for every $V \in \operatorname{Rep}\left(T^{\sharp}\right)$ and $\mathcal{M} \in \operatorname{Rep}_{q}(\check{T})$, the square of the braiding

$$
\operatorname{Frob}_{q, T}^{*}(V) \otimes W \rightarrow W \otimes \operatorname{Frob}_{q, T}^{*}(V) \rightarrow \operatorname{Frob}_{q, T}^{*}(V) \otimes W
$$

is the identity map.

4.5.2. Let $\mathcal{C}^{0}$ be an $\mathbb{E}_{3}$-category (i.e., an algebra object in the category of unital braided monoidal categories). Then the operation of tensor product makes $\operatorname{HopfAlg}\left(\mathrm{C}^{0}\right)$ into a monoidal category. If $\mathrm{e}^{0}$ is a symmetric monoidal category, then $\operatorname{HopfAlg}\left(\mathrm{C}^{0}\right)$ acquires a symmetric monoidal structure.

In particular, it makes sense to talk about coassociative (resp., cocommutative) coalgebra objects in $\operatorname{HopfAlg}\left(\varrho^{0}\right)$.

Note that in the commutative case, a structure on a Hopf algebra $H^{0}$ of cocommutative coalgebra object in $\operatorname{HopfAlg}\left(\mathrm{C}^{0}\right)$ is equivalent to the structure on $H^{0}$ of cocommutative Hopf algebra. 
4.5.3. Let $\mathcal{C}$ be a braided monoidal category; let $\mathrm{C}^{0}$ be an $\mathbb{E}_{3}$-category and let us be given a functor

$$
\epsilon: \mathcal{C}^{0} \rightarrow Z_{\mathbb{E}_{3}}(\mathcal{C}) .
$$

In this case, the operation of tensor product defines an action of $\operatorname{HopfAlg}\left(\complement^{0}\right)$ on $\operatorname{HopfAlg}(\mathcal{C})$

$$
H^{0}, H \mapsto \epsilon\left(H^{0}\right) \otimes H .
$$

In particular, it makes sense to talk about a coaction of a coassociative algebra object $H^{0} \in$ $\operatorname{HopfAlg}\left(\mathcal{C}^{0}\right)$ on $H \in \operatorname{HopfAlg}(\mathcal{C})$.

4.5.4. Assume that $\mathrm{e}^{0}$ has a t-structure, so that the operation of tensor product is t-exact. Let $H^{0}$ be a Hopf algebra in the heart of the t-structure. Then the structure on $H^{0}$ of coassociative coalgebra object in $\operatorname{HopfAlg}\left(\mathrm{C}^{0}\right)$ is equivalent to the condition that the square

commutes.

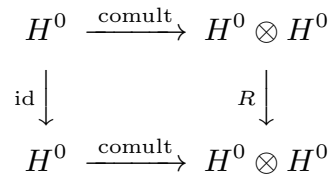

Let $\mathcal{C}$ be also equipped with a t-structure for which the tensor product and the action of $\mathcal{C}^{0}$ on $\mathcal{C}$ are t-exact functors.

Let $H^{0}$ be as above. Let $H$ be a Hopf algebra in $\mathcal{C}$ that also lies in the heart of the t-structure.

Then the datum of coaction of $H^{0}$ on $H$ is equivalent to that of co-central homomorphism

$$
H \rightarrow \epsilon\left(H^{0}\right),
$$

i.e., this is a homomorphism of Hopf algebras that makes the diagram

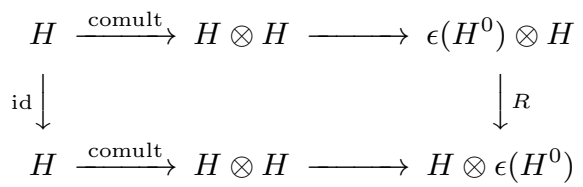

commute.

4.5.5. We now recall that Lusztig's quantum Frobenius is a cocentral map

$$
\operatorname{Frob}_{q, N}: U_{q}^{\mathrm{Lus}}(\check{N}) \rightarrow \operatorname{Frob}_{q, T}^{*}\left(U\left(\mathfrak{n}^{\sharp}\right)\right) .
$$

A basic feature of this map is that the composite map

$$
u_{q}(\check{N}) \rightarrow U_{q}^{\mathrm{Lus}}(\check{N}) \rightarrow \operatorname{Frob}_{q, T}^{*}\left(U\left(\mathfrak{n}^{\sharp}\right)\right)
$$

factors through augmentation, i.e., equals

$$
u_{q}(\check{N}) \stackrel{\text { counit }}{\longrightarrow} \mathrm{e} \stackrel{\text { unit }}{\longrightarrow} \operatorname{Frob}_{q, T}^{*}\left(U\left(\mathfrak{n}^{\sharp}\right)\right) .
$$

Moreover, the resulting map

$$
U_{q}^{\text {Lus }}(\check{N}) \underset{u_{q}(\check{N})}{\otimes} \mathrm{e} \rightarrow \operatorname{Frob}_{q, T}^{*}\left(U\left(\mathfrak{n}^{\sharp}\right)\right)
$$

(as plain objects of $\operatorname{Rep}_{q}(\check{T})$ ), is an isomorphism 14. This expresses the fact that (4.30) is a "short exact sequence" of Hopf algebras.

Remark 4.5.6. We emphasize that it is for the validity of the above assertions that we made the assumption that $q$ should avoid small torsion.

\footnotetext{
${ }^{14}$ The latter fact follows by considering PBW bases.
} 
4.5.7. The maps in (4.30) induce maps

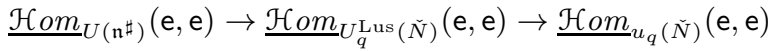

(as associative algebras in $\left.\operatorname{Rep}_{q}(\check{T})\right)$ such that the composite factors through augmentation.

In particular, we obtain a map

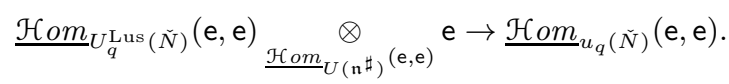

Proposition 4.5.8. The map (4.33) is an isomorphism.

4.5.9. In order to prove Proposition 4.5.8 we will now review the theory of Koszul duality.

Let $A$ be an associative algebra in $\operatorname{Rep}_{q}(\check{T})$, with coweights in $\Lambda^{\text {pos }}$, and such that its coweight 0 component is e (which automatically gives $A$ an augmentation).

Let $A$-mod ${ }^{\text {loc.nilp }}\left(\operatorname{Rep}_{q}(\check{T})\right)$ be the category of locally nilpotent $A$-modules in $\operatorname{Rep}_{q}(\check{T})$. By definition, this category is compactly generated by modules of the form $\mathrm{e}^{\lambda}$ with the trivial action. Set

$$
B:=\underline{\mathcal{H} o m}_{A}(\mathrm{e}, \mathrm{e})^{\mathrm{op}} .
$$

Then the functor

$$
\mathcal{M} \mapsto \underline{\mathcal{H} o m}_{A}(\mathrm{e}, \mathcal{M})
$$

defines an equivalence

$$
\mathrm{KD}_{A}: A-\bmod ^{\text {loc.nilp }}\left(\operatorname{Rep}_{q}(\check{T})\right) \rightarrow B-\bmod \left(\operatorname{Rep}_{q}(\check{T})\right)
$$

We have

$$
\mathrm{KD}_{A}(\mathrm{e}) \simeq B \text { and } \mathrm{KD}_{A}\left(A^{\vee}\right) \simeq \mathrm{e}
$$

where $A^{\vee}$ denotes the $\left(\Lambda\right.$-graded) dual of $A$, naturally considered as an object of $A$-mod ${ }^{\text {loc.nilp }}\left(\operatorname{Rep}_{q}(\check{T})\right)$.

4.5.10. Let now

$$
\epsilon_{A}: A_{1} \rightarrow A_{2}
$$

be a homomorphism of associative algebras. We have the forgetful functor

$$
\operatorname{oblv}_{A_{2} \rightarrow A_{1}}: A_{2}-\bmod ^{\text {loc.nilp }}\left(\operatorname{Rep}_{q}(\check{T})\right) \rightarrow A_{2}-\bmod ^{\text {loc.nilp }}\left(\operatorname{Rep}_{q}(\check{T})\right),
$$

and its right adjoint, denoted coind ${ }_{A_{1} \rightarrow A_{2}}$, and given by

$$
\mathcal{M}_{1} \mapsto \underline{\mathcal{H} o m}_{A_{1}}\left(A_{2}, \mathcal{M}_{1}\right)
$$

The homomorphism $\epsilon_{A}$ gives rise to a homomorphism

$$
\epsilon_{B}: B_{2} \rightarrow B_{1}
$$

Under the equivalences (4.34) for $A_{1}$ and $A_{2}$, the functors oblv $A_{2} \rightarrow A_{1}$ and coind A $_{1} \rightarrow A_{2}$ correspond to the functors

$$
\operatorname{ind}_{B_{2} \rightarrow B_{1}}: B_{2}-\bmod \left(\operatorname{Rep}_{q}(\check{T})\right) \rightleftarrows B_{1}-\bmod \left(\operatorname{Rep}_{q}(\check{T})\right): \mathbf{o b l v}_{B_{1} \rightarrow B_{2}},
$$

where $\operatorname{ind}_{B_{2} \rightarrow B_{1}}$ is the functor

$$
\mathcal{N}_{2} \mapsto B_{1} \underset{B_{2}}{\otimes} \mathcal{N}_{2}
$$


4.5.11. We are now ready to prove Proposition 4.5.8

Let us apply the discussion in Sect.4.5.10 to the homomorphism

$$
U_{q}^{\text {Lus }}(\check{N}) \rightarrow \operatorname{Frob}_{q, T}^{*}\left(U\left(\mathfrak{n}^{\sharp}\right)\right) .
$$

We obtain that under the equivalence

$$
\underline{\mathcal{H} o m}_{U_{q}^{\mathrm{Lus}}(\check{N})}(\mathrm{e}, \mathrm{e})-\bmod \simeq U_{q}^{\mathrm{Lus}}(\check{N})-\bmod ^{\text {loc.nilp }},
$$

the left-hand side in 4.33 corresponds to

$$
\operatorname{Frob}_{q, T}^{*}\left(\left(U\left(\mathfrak{n}^{\sharp}\right)\right)^{\vee}\right),
$$

viewed as a $U_{q}^{\text {Lus }}(\check{N})$-module via $\operatorname{Frob}_{q, N}$.

Consider now the homomorphism

$$
u_{q}(\check{N}) \rightarrow U_{q}^{\text {Lus }}(\check{N})
$$

We obtain that the right-hand side in (4.33), viewed as a $\underline{\mathcal{H} o m}_{U_{q}^{\operatorname{Lus}}(\check{N})}(\mathrm{e}, \mathrm{e})$-module via

$$
\underline{\mathcal{H} o m}_{U_{q}^{\operatorname{Lus}(\check{N})}}(\mathrm{e}, \mathrm{e}) \rightarrow \underline{\mathcal{H} o m}_{u_{q}(\check{N})}(\mathrm{e}, \mathrm{e}),
$$

corresponds under 4.35) to

$$
\underline{\mathcal{H} o m}_{u_{q}(\check{N})}\left(U_{q}^{\mathrm{Lus}}(\check{N}), \mathrm{e}\right) .
$$

Hence, the isomorphism stated in the proposition amounts to the fact that the map

$$
\operatorname{Frob}_{q, T}^{*}\left(\left(U\left(\mathfrak{n}^{\sharp}\right)\right)^{\vee}\right) \rightarrow \underline{\mathcal{H} o m}_{u_{q}(\check{N})}\left(U_{q}^{\text {Lus }}(\check{N}), \text { e }\right)
$$

is an isomorphism.

However, this follows from (4.31) by dualization.

\subsection{Quantum Frobenius for factorization algebras.}

4.6.1. In this section we will perform the construction of the action of $\Omega^{\sharp, c l}$ on $\Omega_{q, \text { Quant }}^{\mathrm{Lus}}$.

This is obtained by enhancing the paradigm of Sect. 3.4

4.6.2. First, the (symmetric) monoidal structure on $\operatorname{Hopf} \operatorname{Alg}\left(\operatorname{Rep}\left(T^{\sharp}\right)\right)$

$$
H_{1}^{0}, H_{2}^{0} \mapsto H_{1}^{0} \otimes H_{2}^{0}
$$

corresponds under the equivalence (3.15) to the (symmetric) monoidal structure on the category of factorization algebras in $\operatorname{Shv}\left(\left(\mathrm{Conf}^{\sharp}\right)^{+}\right)$given by convolution:

$$
\mathcal{A}_{1}, \mathcal{A}_{2} \mapsto \mathcal{A}_{1} \star \mathcal{A}_{2},
$$

see Remark 4.2 .2

Further, the action of $\operatorname{HopfAlg}\left(\operatorname{Rep}\left(T^{\sharp}\right)\right)$ on $\operatorname{HopfAlg}\left(\operatorname{Rep}_{q}(\check{T})\right)$ corresponds under the equivalence (3.15) to the action of the category of factorization algebras in $\operatorname{Shv}\left(\operatorname{Conf}^{\sharp}\right)$ on the category of factorization algebras in $\mathrm{Shv}_{\mathcal{G}_{q}^{\text {Conf }}}(\mathrm{Conf})$, also given by convolution:

$$
\mathcal{A}^{\sharp}, \mathcal{A} \mapsto \mathcal{A}^{\sharp} \star \mathcal{A} .
$$

4.6.3. Hence, the datum of the co-central homomorphism (4.29) gives rise to an action of $\Omega^{\sharp, c l}$ on $\Omega_{q, \text { Quant }}^{\text {Lus }}$.

Finally, the isomorphism (4.25) follows from the isomorphism of Proposition 4.5.8 (see Remark 4.4 .3 .

\section{ZASTAVA SPACES AND WHITTAKER/SEMI-INFINITE CATEGORIES}

In this section we review some geometric constructions needed in order to introduce the Whittaker incarnations of the factorization algebras $\Omega_{q}^{\text {? }}$.

\subsection{Affine Grassmannian over the configuration space.}


5.1.1. Recall that we denote by $G$ the semi-simple simply connected group, whose coroot lattice is $\Lambda$. Let

$$
\mathrm{Gr}_{G, \mathrm{Conf}}^{\omega^{\rho}} \rightarrow \text { Conf }
$$

denote the following version of the affine Grassmannian of $G$ :

Namely, for a test-scheme $Y$, a $Y$-point of $\operatorname{Gr}_{G \text {, Conf }}^{\omega^{\rho}}$ is a triple $\left(D, \mathcal{P}_{G}, \alpha\right)$, where:

- $D$ is a $Y$-point of Conf;

- $\mathcal{P}_{G}$ is a $G$-bundle on $Y \times X$;

- $\alpha$ is an identification of $\mathcal{P}_{G}$ with the $G$-bundle $\mathcal{P}_{G}^{\omega^{\rho}}$, defined on $Y \times X-\Gamma_{D}$.

Here:

- $\mathcal{P}_{G}^{\omega^{\rho}}$ is the $G$-bundle induced from a (chosen once and for all) square root $\omega^{\otimes \frac{1}{2}}$ of the canonical line bundle $\omega$ via

$$
\mathbb{G}_{m} \stackrel{2 \rho}{\longrightarrow} T \hookrightarrow G
$$

- $\Gamma_{D}$ is the preimage of the incidence divisor in Conf $\times X$ under

$$
Y \times X \stackrel{D \times \text { id }}{\rightarrow} \text { Conf } \times X .
$$

We let

$$
\mathfrak{s}: \text { Conf } \rightarrow \operatorname{Gr}_{G, \text { Conf }}^{\omega^{\rho}}
$$

denote the unit section, which sends $D$ to the triple $\left(D, \mathcal{P}_{G}^{\omega^{\rho}}\right.$, id).

5.1.2. Along with $\mathrm{Gr}_{G \text {, Conf }}^{\omega^{\rho}}$ one introduces the corresponding version of the group-(ind)schemes

$$
\mathfrak{L}^{+}(G)_{\text {Conf }}^{\omega^{\rho}} \subset \mathfrak{L}(G)_{\text {Conf }}^{\omega^{\rho}}
$$

see GLys2, Sect. 12.2.1], equipped with an action on $\operatorname{Gr}_{G \text {, Conf }}^{\omega^{\rho}}$.

We can identify $\operatorname{Gr}_{G \text {, Conf }}^{\omega^{\rho}}$ with the prestack quotient

$$
\mathfrak{L}(G)_{\text {Conf }}^{\omega^{\rho}} / \mathfrak{L}^{+}(G)_{\text {Conf }}^{\omega^{\rho}},
$$

sheafified in the étale topology.

5.1.3. A key feature of $\operatorname{Gr}_{G, \text { Conf }}^{\omega^{\rho}}$ is the factorization structure over Conf, i.e., a canonical isomorphism

$$
\left(\operatorname{Gr}_{G, \text { Conf }}^{\omega^{\rho}} \times \operatorname{Gr}_{G, \text { Conf }}^{\omega^{\rho}}\right) \underset{\text { Conf } \times \text { Conf }}{\times}(\text { Conf } \times \text { Conf })_{\text {disj }} \simeq \operatorname{Gr}_{G, \text { Conf }}^{\omega^{\rho}} \underset{\mathrm{Conf}}{\times}(\text { Conf } \times \text { Conf })_{\operatorname{disj}},
$$

which is associative in the natural sense.

The same applies to the group-(ind)schemes $\mathfrak{L}(G){ }_{\text {Conf }}^{\omega^{\rho}}$ and $\mathfrak{L}^{+}(G)_{\text {Conf }}^{\omega^{\rho}}$.

5.1.4. Inside $\mathfrak{L}(G)_{\text {Conf }}^{\omega^{\rho}}$ we consider the group ind-subscheme

$$
\mathfrak{L}(N)_{\text {Conf }}^{\omega^{\rho}} \subset \mathfrak{L}(G)_{\text {Conf }}^{\omega^{\rho}} .
$$

The purpose of the twist $\omega^{\rho}$ was that there exists a canonical character

$$
\chi: \mathfrak{L}(N)_{\text {Conf }}^{\omega^{\rho}} \rightarrow \mathbb{G}_{a},
$$

see GLys2, Sect. 8.2.1].

The character $\chi$ is compatible with the factorization structure in the natural sense.

5.1.5. It follows from [GLys1, Sect. 3.3.4 and Corollary 3.3.6] that the form $q$ gives rise to a canonically defined geometric metaplectic datum, for $G$ (here we use the assumption that $G$ is simply-connected).

In particular, by GLys2, Sect. 4.6.3], we obtain a gerbe on $\operatorname{Gr}_{G \text {, Conf }}^{\omega^{\rho}}$, denoted $\mathcal{G}_{q}^{G}$, equipped with a factorization structure.

\subsection{Semi-infinite orbits and their closures.}


5.2.1. In what follows we will consider the closed subfunctor

$$
\bar{S}_{\text {Conf }}^{0} \subset \operatorname{Gr}_{G, \text { Conf }}^{\omega^{\rho}}
$$

and its open subfunctor

$$
S_{\text {Conf }}^{0} \subset \bar{S}_{\text {Conf }}^{0}
$$

Namely, $\bar{S}_{\text {Conf }}^{0}$ consists of those triples $\left(D, \mathcal{P}_{G}, \alpha\right)$, for which for every dominant weight $\check{\lambda}$, the map

$$
\omega^{\otimes\langle\rho, \check{\lambda}\rangle} \rightarrow \mathcal{V}_{\mathcal{P}}^{\check{\lambda}} \omega^{\check{\lambda}} \rightarrow \mathcal{V}_{\mathcal{P}_{G}}^{\lambda},
$$

defined away from $\Gamma_{D}$, extends to all of $Y \times X$.

In the above formula:

- $V^{\check{\lambda}}$ denotes the Weyl module for $G$ of highest weight $\check{\lambda}$;

- $\mathcal{V}_{\mathcal{P}_{G}}^{\check{\lambda}}$ is the vector bundle, obtained as the twist of $\mathcal{V}^{\lambda}$ by the given $G$-bundle;

- $\omega^{\otimes\langle\rho, \check{\lambda}\rangle}:=\left(\omega^{\otimes \frac{1}{2}}\right)^{\otimes\langle 2 \rho, \check{\lambda}\rangle}$;

- The map $\omega^{\otimes\langle\rho, \check{\lambda}\rangle} \rightarrow \mathcal{V}_{\mathcal{P}}^{\check{\lambda} \omega}$ is given by the highest weight line in $\mathcal{V}^{\check{\lambda}}$.

5.2.2. We let

$$
S_{\text {Conf }}^{0} \stackrel{\mathbf{j}}{\longrightarrow} \bar{S}_{\mathrm{Conf}}^{0}
$$

be the locus corresponding to the condition that (5.2) are injective bundle maps, i.e., that the quotient is a vector bundle on $Y \times X$.

The subfunctors

$$
S_{\mathrm{Conf}}^{0} \subset \bar{S}_{\mathrm{Conf}}^{0} \subset \mathrm{Gr}_{G, \mathrm{Conf}}^{\omega^{\rho}}
$$

inherit a factorization structure from that of $\operatorname{Gr}_{G, \text { Conf }}^{\omega^{\rho}}$.

5.2.3. Both subfunctors (5.3) are preserved by the action of $\mathfrak{L}(N) \omega_{\text {Conf }}^{\omega^{\rho}}$. Moreover, the action of $\mathfrak{L}(N){ }_{\text {Conf }}^{\omega^{\rho}}$ on the unit section

$$
\mathfrak{s}: \text { Conf } \rightarrow S_{\text {Conf }}^{0} \subset \mathrm{Gr}_{G, \mathrm{Conf}}^{\omega^{\rho}}
$$

defines an isomorphism

$$
\mathfrak{L}(N)_{\text {Conf }}^{\omega^{\rho}} / \mathfrak{L}^{+}(N)_{\text {Conf }}^{\omega^{\rho}} \simeq S_{\text {Conf }}^{0},
$$

From here we obtain that the character $\chi$ induces a map

$$
S_{\text {Conf }}^{0} \rightarrow \mathbb{G}_{a}
$$

which we will denote by the same character $\chi$.

5.2.4. The gerbe $\mathcal{G}_{q}^{G}$ is equivariant with respect to the action of $\mathfrak{L}(N)_{\text {Conf }}^{\omega^{\rho}}$ on $\operatorname{Gr}_{G}^{\omega^{\rho} \text {, Conf }}$. Moreover, for any point of $\operatorname{Gr}_{G \text {, Conf }}^{\omega^{\rho}}$, the resulting character sheaf on its stabilizer in $\mathfrak{L}(N){ }_{\text {Conf }}^{\omega^{\rho}}$ is trivial.

In particular, the restriction

$$
\left.\mathcal{G}_{q}^{G}\right|_{S_{\mathrm{Conf}}^{0}}
$$

admits a canonical $\mathfrak{L}(N)_{\text {Conf-equivariant trivialization. }}^{\omega^{\rho}}$ 
5.2.5. Let

$$
\bar{S}_{\text {Conf }}^{-, \text {Conf }} \subset \operatorname{Gr}_{G, \text { Conf }}^{\omega^{\rho}}
$$

be the closed subfunctor defined as follows: a $Y$-point $\left(D, \mathcal{P}_{G}, \alpha\right)$ of $\operatorname{Gr}_{G}^{\omega^{\rho} \text {, Conf }}$ belongs to $\bar{S}_{\text {Conf }}^{-, \text {Conf }}$ if for every dominant weight $\check{\lambda}$, the composite map

$$
\mathcal{V}_{\mathcal{P}_{G}}^{\vee, \lambda} \simeq \mathcal{V}_{\mathcal{P}}^{\vee, \check{\lambda} \rho} \underset{G}{\vee \sim} \rightarrow \omega^{\otimes\langle\rho, \check{\lambda}\rangle}
$$

which defined away from $\Gamma_{D}$, extends to a regular map

$$
\mathcal{V}_{\mathcal{P}_{G}}^{\vee, \lambda} \rightarrow \omega^{\otimes\langle\rho, \check{\lambda}\rangle}(-\check{\lambda}(D)),
$$

where

- $\mathcal{V}^{\vee, \check{\lambda}}$ denotes the dual Weyl module for $G$ of highest weight $\check{\lambda}$;

- The map $\mathcal{V}_{\mathcal{P}}^{\vee, \tilde{\lambda} \rho} \rightarrow \omega^{\otimes\langle\rho, \check{\lambda}\rangle}$ corresponds to the canonical $N^{-}$-invariant functional on $\mathcal{V}^{\vee, \check{\lambda}}$;

- $-\check{\lambda}(D)$ is the effective (!) Cartier divisor on $Y \times X$ equal to the pullback of the tautological effective divisor on Div ${ }^{\text {eff }} \times X$ along the map

$$
Y \times X \stackrel{D \times \text { id }}{\longrightarrow} \text { Conf } \times X \stackrel{-\check{\lambda} \times \text { id }}{\longrightarrow} \operatorname{Div}^{\text {eff }} \times X
$$

5.2.6. Let

$$
S_{\text {Conf }}^{-, \text {Conf }} \stackrel{\mathbf{j}^{-}}{\longrightarrow} \bar{S}_{\mathrm{Conf}}^{-, \mathrm{Conf}}
$$

be the open subfunctor corresponding to the locus, where the maps (5.6) are regular bundle maps.

5.2.7. Note that in addition to the unit section

$$
\mathfrak{s}: \text { Conf } \rightarrow \operatorname{Gr}_{G, \text { Conf }}^{\omega^{\rho}}
$$

there exists another canonical section

$$
\mathfrak{s}^{-}: \text {Conf } \rightarrow \operatorname{Gr}_{G, \operatorname{Conf}}^{\omega^{\rho}}
$$

that sends a $Y$-point of Conf, given by $D$, to the $G$-bundle induced from the $T$-bundle

$$
\check{\lambda} \mapsto \omega^{\otimes\langle\rho, \check{\lambda}\rangle}(-\check{\lambda}(D)) .
$$

This image of this section belongs to $S_{\text {Conf }}^{-, \text {Conf }}$.

The action of $\mathfrak{L}\left(N^{-}\right) \omega_{\text {Conf }}^{\rho}$ on this section defines an isomorphism

$$
\mathfrak{L}\left(N^{-}\right)_{\text {Conf }}^{\omega^{\rho}} / \operatorname{Ad}_{\mathfrak{s}^{-}}\left(\mathfrak{L}^{+}\left(N^{-}\right)_{\text {Conf }}^{\omega^{\rho}}\right) \rightarrow S_{\text {Conf }}^{-, \text {Conf }} .
$$

5.2.8. The gerbe $\mathcal{G}_{q}^{G}$ is equivariant also with respect to $\mathfrak{L}\left(N^{-}\right)_{\text {Conf }}^{\omega^{\rho}}$, and for any point of $\operatorname{Gr}_{G}^{\omega^{\rho}}$, Conf , the resulting character sheaf on its stabilizer in $\mathfrak{L}\left(N^{-}\right){ }_{\text {Conf }}^{\omega^{\rho}}$ is trivial.

Moreover, according to GLys1, Sect. 5.1], the pullback of $\mathcal{G}_{q}^{G}$ along $\mathfrak{s}^{-}$identifies canonically with $\mathcal{G}_{q}^{\text {Conf }}$.

From here it follows that the restriction of $\mathcal{G}_{q}^{G}$ to $S_{\text {Conf }}^{-, \text {Conf }}$ carries a (unique) $\mathfrak{L}\left(N^{-}\right)_{\text {Conf }}^{\omega^{\rho} \text {-equivariant }}$ identification with the pullback of the gerbe $\mathcal{G}_{q}^{\text {Conf }}$ along

$$
S_{\text {Conf }}^{-, \text {Conf }} \rightarrow \text { Conf. }
$$


5.2.9. Let $\mathcal{G}_{q}^{\text {ratio }}$ denote the gerbe on $\mathrm{Gr}_{G, \text { Conf }}^{\omega^{\rho}}$ equal to the ratid 15

$$
\mathcal{G}_{q}^{\text {ratio }}:=\mathcal{G}_{q}^{\text {Conf }} \otimes\left(\mathcal{G}_{q}^{G}\right)^{-1},
$$

where, by a slight abuse of notation, we denote by $\mathcal{G}_{q}^{\text {Conf }}$ the pullback of the same-named gerbe along

$$
\mathrm{Gr}_{G, \mathrm{Conf}}^{\omega^{\rho}} \rightarrow \text { Conf. }
$$

We obtain that the restriction of $\mathcal{G}_{q}^{\text {ratio }}$ to $S_{\text {Conf }}^{- \text {,Conf }}$ admits a canonical $\mathfrak{L}\left(N^{-}\right)_{\text {Conf-equivariant trivi- }}^{\omega^{\rho}}$ alization.

5.3. Zastava spaces. In this subsection we recall the definition of the (several versions of the) Zastava space.

5.3.1. The (compactified) Zastava space $\bar{z}$ is defined as

$$
\bar{z}:=\bar{S}_{\text {Conf }}^{0} \cap \bar{S}_{\text {Conf }}^{-, \text {Conf }} \subset \operatorname{Gr}_{G, \text { Conf }}^{\omega^{\rho}}
$$

Let $\pi$ denote the projection $\bar{z} \rightarrow$ Conf.

A priori, $\bar{z}$ is an ind-scheme, but one shows (using global considerations, see GLys2, Corollary 20.2.3]) that $\bar{z}$ is actually a scheme. Since $\operatorname{Gr}_{G}^{\omega^{\rho} \text {,Conf }}$ is ind-proper over Conf, we obtain that the natural projection

$$
\bar{\pi}: \bar{z} \rightarrow \text { Conf }
$$

is proper.

5.3.2. The functor represented by $\bar{z}$ can be explicitly described as follows. For a test-scheme $Y$, a $Y$-point of $\bar{z}$ is a triple

$$
\left(D, \mathcal{P}_{G},\{\kappa\},\left\{\kappa^{-}\right\}\right),
$$

where

- $D$ is a $Y$-valued point of Conf;

- $\mathcal{P}_{G}$ is a $G$-bundle on $Y \times X$;

- $\{\kappa\}$ is a collection of maps

$$
\omega^{\otimes\langle\rho, \check{\lambda}\rangle} \rightarrow V_{\mathcal{P}_{G}}^{\check{\lambda}}, \quad \check{\lambda} \in \check{\Lambda}^{+}
$$

satisfying the Plücker relations;

- $\left\{\kappa^{-}\right\}$is a collection of maps

$$
\mathcal{V}_{\mathcal{P}_{G}}^{\vee, \check{\lambda}} \rightarrow \omega^{\otimes\langle\rho, \check{\lambda}\rangle}(-\check{\lambda}(D)), \quad \check{\lambda} \in \check{\Lambda}^{+},
$$

satisfying the Plücker relations.

We require that:

- the composite maps

$$
\omega^{\otimes\langle\rho, \check{\lambda}\rangle} \rightarrow \mathcal{V}_{\mathcal{P}_{G}}^{\check{\lambda}} \rightarrow \mathcal{V}_{\mathcal{P}_{G}, \check{\lambda}}^{\vee} \rightarrow \omega^{\otimes\langle\rho, \check{\lambda}\rangle}(-\check{\lambda}(D))
$$

are the tautological maps

$$
\omega^{\otimes\langle\rho, \check{\lambda}\rangle} \rightarrow \omega^{\otimes\langle\rho, \check{\lambda}\rangle}(-\check{\lambda}(D)) .
$$

\footnotetext{
${ }^{15}$ The notation diverges from one in GLys2 by replacing $q$ by $q^{-1}$.
} 
5.3.3. We define the open subfunctors

to be

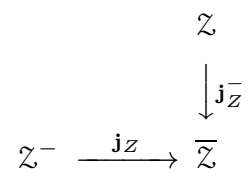

$$
z:=\bar{S}_{\mathrm{Conf}}^{0} \cap S_{\mathrm{Conf}}^{-, \mathrm{Conf}} \text { and } z^{-}:=S_{\mathrm{Conf}}^{0} \cap \bar{S}_{\mathrm{Conf}}^{-, \operatorname{Conf}}
$$

respectively.

These subschemes correspond to the condition that the maps (5.11) (resp., (5.10) are injective bundle maps.

Let $\pi$ (resp., $\pi^{-}$) denote the restriction of $\bar{\pi}$ to $z$ (resp., $z^{-}$).

Note that since the maps

$$
S_{\text {Conf }}^{0} \rightarrow \text { Conf } \leftarrow S_{\text {Conf }}^{-, \text {Conf }}
$$

are ind-affine, the maps

$$
z \stackrel{\pi}{\rightarrow} \text { Conf } \stackrel{\pi^{-}}{\leftarrow} z^{-}
$$

are affine.

5.3.4. Set also

$$
\stackrel{\circ}{z}:=z \cap z^{-} \subset \bar{z}
$$

We have the corresponding open embeddings

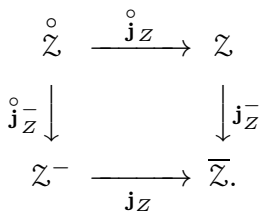

Let $\stackrel{\circ}{\pi}$ denote the restriction of $\pi$ to $\stackrel{\circ}{z}$. This map is also affine, as are the maps $\stackrel{\circ}{\mathbf{j}} z$ and $\stackrel{\circ}{\mathbf{j}}_{Z}^{-}$.

It is known that $\stackrel{\circ}{z}$ is a smooth scheme; its connected component living over $\operatorname{Conf}^{\lambda} \subset$ Conf has $^{2}$ dimension $-\langle\lambda, 2 \check{\rho}\rangle$.

5.3.5. We let $\mathcal{G}_{q}^{G}$ denote the restriction of the same-named gerbe along

$$
\bar{z} \hookrightarrow \operatorname{Gr}_{G, \text { Conf } .}
$$

Let $\mathcal{G}_{q}^{\text {Conf }}$ denote the pullback of the same-named gerbe along $\bar{\pi}$. Let $\mathcal{G}_{q}^{\text {ratio }}$ denote the ratio of the latter by the former.

We obtain that

$$
\left.\mathcal{G}_{q}^{G}\right|_{z^{-}} \text {and }\left.\mathcal{G}_{q}^{\text {ratio }}\right|_{z}
$$

admit canonical trivializations.

5.3.6. The spaces

$$
\stackrel{\circ}{z}, z, z^{-}, \bar{z}
$$

all inherit a factorization structure from that on $\mathrm{Gr}_{G \text {, Conf }}^{\omega^{\rho}}$, along with the gerbes

$$
\mathcal{G}_{q}^{G}, \mathcal{G}_{q}^{\text {Conf }}, \mathcal{G}_{q}^{\text {ratio }}
$$

\subsection{Sheaves on Zastava spaces.}


5.4.1. The restriction of the map

$$
\chi: S_{\text {Conf }}^{0} \rightarrow \mathbb{G}_{a}
$$

along $Z^{-} \hookrightarrow S_{\text {Conf }}^{0}$ defines a map

$$
z^{-} \rightarrow \mathbb{G}_{a}
$$

which we denote by the same symbol $\chi$.

Let

$$
\operatorname{Vac}_{\text {Whit }, Z} \in \operatorname{Shv}\left(z^{-}\right)
$$

denote the object

$$
\omega_{z-} \stackrel{*}{\otimes} \chi^{*}(\exp ),
$$

where exp denotes the exponential/Artin-Schreier local system on $\mathbb{G}_{a}$.

The object Vacwhit, $Z$ is equipped with a factorization structure along Conf in a natural sense.

We will refer to $\operatorname{Vac}_{W h i t, Z}$ as the basic (a.k.a. vacuum Whittaker) sheaf on $z^{-}$.

5.4.2. Due to the trivialization of $\left.\mathcal{G}_{q}^{G}\right|_{Z^{-}}$, we can think of Vacwhit, $Z$ as an object of the twisted category $\operatorname{Shv}_{\mathcal{G}_{q}}\left(Z^{-}\right)$, and in this capacity we will denote it by

$$
\operatorname{Vac}_{q, \text { Whit }, Z} \in \operatorname{Shv}_{\mathcal{G}_{q}^{G}}\left(Z^{-}\right) \text {. }
$$

This object is also equipped with a factorization structure along Conf.

5.4.3. Consider the category $\operatorname{Shv}(\stackrel{\circ}{Z})$ and the object

$$
\mathrm{IC}_{\stackrel{z}{ }} \in \operatorname{Shv}(\stackrel{\circ}{Z})
$$

Note, however, that since $\stackrel{\circ}{Z}$ is smooth,

$$
\mathrm{IC}_{\mathrm{z}} \simeq \omega_{\mathrm{z}}[-\mathrm{deg}]
$$

where deg takes value $-\langle\lambda, 2 \check{\rho}\rangle$ over the connected component $\operatorname{Conf}^{\lambda}$ of Conf.

Due to the trivialization of $\left.\mathcal{G}_{q}^{\text {ratio }}\right|_{z}$, we can think of $\mathrm{IC}_{\odot}$ as an object of the category $\operatorname{Shv}_{\mathcal{G}_{q}^{\text {ratio }}}(\stackrel{\circ}{\mathcal{Z}})$. When viewed in this capacity, we will denote it by

$$
\mathrm{IC}_{q, \stackrel{\mathcal{Z}}{ }} \in \operatorname{Shv}_{\mathcal{G}_{q}^{\text {ratio }}}(\stackrel{\circ}{\bar{z}}) .
$$

We will consider several variants of its extension to an object of $\operatorname{Shv}_{\mathcal{G}_{q}^{\text {ratio }}}\left(Z^{-}\right)$:

$$
\begin{aligned}
& \nabla_{q, Z}^{-}:=\left(\stackrel{\circ}{\mathbf{j}}_{Z}^{-}\right)_{*}\left(\mathrm{IC}_{q, \stackrel{\circ}{z}}\right), \quad \Delta_{q, Z}^{-}:=\left(\stackrel{\circ}{Z}_{Z}^{-}\right) !\left(\mathrm{IC}_{q, \stackrel{\circ}{z}}\right), \\
& \mathrm{IC}_{q, Z}^{-}:=\left(\stackrel{\circ}{\mathbf{j}}_{Z}^{-}\right) ! *\left(\mathrm{IC}_{q, \stackrel{\circ}{z}}\right) .
\end{aligned}
$$

We have the tautological maps

$$
\Delta_{q, Z}^{-} \rightarrow \mathrm{IC}_{q, Z}^{-} \rightarrow \nabla_{q, Z}^{-}
$$

We have the following result (see [Ga3, Theorem 7.6]):

Proposition 5.4.4. For $q$ which is non-torsion valued, the maps (5.12) are isomorphisms. 
5.4.5. Set 16

$$
\begin{gathered}
\operatorname{Gauss}_{q, *}^{-}:=\operatorname{Vac}_{\text {Whit }, Z} \stackrel{!}{\otimes} \nabla_{q, Z}^{-}, \quad \text { Gauss }_{q, !}^{-}:=\operatorname{Vac}_{W h i t, Z} \stackrel{!}{\otimes} \Delta_{q, Z}^{-}, \\
\text {Gauss }_{q, ! *}^{-}:=\text {Vac Whit }, Z^{\prime} \stackrel{\mathrm{IC}_{q, Z}^{-} .}{ }
\end{gathered}
$$

The above three are objects of the category

$$
\operatorname{Shv}_{\mathcal{G}_{q} \operatorname{Conf}}\left(Z^{-}\right)
$$

where we are using $\stackrel{!}{\otimes}$ as a functor

$$
\operatorname{Shv}_{\mathcal{G}_{q}^{G}}\left(z^{-}\right) \times \operatorname{Shv}_{\mathcal{G}_{q}^{\text {ratio }}}\left(Z^{-}\right) \rightarrow \operatorname{Shv}_{\mathcal{G}_{q}^{\text {Conf }}}\left(Z^{-}\right)
$$

All three are perverse sheaves, and they each identify with the !-, *- and !*-extension, respectively, of their common restriction to $\stackrel{\circ}{z}$. The maps $(5.12$ induce maps

$$
\text { Gauss }_{q, !}^{-} \rightarrow \text { Gauss }_{q, ! *}^{-} \rightarrow \text { Gauss }_{q, *}^{-} .
$$

According to Proposition 5.4.4 the maps (5.13) are isomorphisms for $q$ which is non-torsion valued.

In addition, we have

$$
\mathbb{D}^{\text {Verdier }}\left(\operatorname{Gauss}_{q, *}^{-}\right) \simeq \operatorname{Gauss}_{q^{-1}, !}^{-} \text {and } \mathbb{D}^{\text {Verdier }}\left(\operatorname{Gauss}_{q, ! *}^{-}\right) \simeq \operatorname{Gauss}_{q^{-1}, ! *}^{-},
$$

up to replacing exp by its inverse.

5.4.6. We now have the following key assertion:

Theorem 5.4.7. The maps

$$
\begin{aligned}
\left(\mathbf{j}_{Z}\right) !\left(\operatorname{Gauss}_{q, *}^{-}\right) & \rightarrow\left(\mathbf{j}_{Z}\right)_{*}\left(\operatorname{Gauss}_{q, *}^{-}\right) \\
\left(\mathbf{j}_{Z}\right) !\left(\operatorname{Gauss}_{q, !}^{-}\right) & \rightarrow\left(\mathbf{j}_{Z}\right)_{*}\left(\operatorname{Gauss}_{q, !}^{-}\right)
\end{aligned}
$$

and

are isomorphisms.

$$
\left(\mathbf{j}_{Z}\right) !\left(\operatorname{Gauss}_{q, ! *}^{-}\right) \rightarrow\left(\mathbf{j}_{Z}\right) *\left(\operatorname{Gauss}_{q, ! *}^{-}\right)
$$

In other words, Theorem 5.4 .7 says that extension of each of the objects $\operatorname{Gauss}_{q, *}^{-}, \operatorname{Gauss}_{q, !}^{-}$and $\operatorname{Gauss}_{q, ! *}^{-}$along

is clean.

$$
\mathbf{j}_{z}: z^{-} \rightarrow \bar{z}
$$

Proof of Theorem 5.4.7. The proof of the assertion concerning Gauss ${ }_{q, ! *}^{-}$repeats that of Ga3, Theorem 7.3], where we replace Ga3, Proposition 7.9] by a more precise reference, namely Camp, Theorem 4.2.1].

The statements concerning $\operatorname{Gauss}_{q, *}^{-}$and $\operatorname{Gauss}_{q, !}^{-}$are Verdier duals of one another, so we will prove the latter.

The assertion concerning Gauss $_{q, !}^{-}$follows from Corollary 6.7.3 below: the object Gauss ${ }_{q, !}^{-}$admits a filtration indexed by $\Lambda^{\text {neg }}$ by objects of the form

$$
\left(\mathbf{i}^{\lambda}\right)_{*}\left(\left.\Omega^{\sharp, c l}\right|_{X^{\lambda}} \otimes \operatorname{Gauss}_{q, ! *}^{-}\right),
$$

where $\mathbf{i}^{\lambda}$ is the (finite) map

$$
X^{\lambda} \times \bar{z} \rightarrow \bar{z}
$$

given by adding the divisor:

$$
\mathbf{i}^{\lambda}\left(D^{\prime},\left(D, \mathcal{P}_{G}, \alpha\right)\right)=\left(D+D^{\prime}, \mathcal{P}_{G}, \alpha\right)
$$

\footnotetext{
${ }^{16}$ See Remark 6.2 .3 for the explanation of the name Gauss.
} 
so that we have a commutative diagram

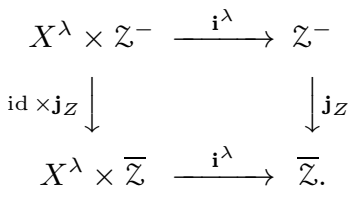

5.4.8. Denote by

$$
\operatorname{Gauss}_{q, *}, \quad \operatorname{Gauss}_{q, !} \text { and } \operatorname{Gauss}_{q, ! *}
$$

the resulting three objects of the category $\operatorname{Shv}_{\mathcal{G}_{q} \text { Conf }}(\overline{\bar{z}})$.

By construction, they carry a natural factorization structure with respect to Conf. The maps (5.13) induce maps

$$
\text { Gauss }_{q, !} \rightarrow \text { Gauss }_{q, ! *} \rightarrow \operatorname{Gauss}_{q, *} .
$$

These maps are isomorphisms for $q$ which is non-torsion valued.

\section{Corollary 5.4.9.}

(a) The objects Gauss $_{,, *}$, Gauss $_{q, !}$ and Gauss $_{q, ! *}$ are perverse, and identify with the *-, !- and GoreskyMacPherson extensions along $\stackrel{\circ}{\mathrm{z}} \hookrightarrow \bar{z}$ of

$$
\chi^{*}(\exp ) \stackrel{*}{\otimes} \mathrm{IC}_{q, \stackrel{\circ}{z}}
$$

(b) We have canonical isomorphisms

$$
\mathbb{D}^{\text {Verdier }}\left(\operatorname{Gauss}_{q, *}\right) \simeq \operatorname{Gauss}_{q^{-1}, !} \text { and } \mathbb{D}^{\text {Verdier }}\left(\operatorname{Gauss}_{q, ! *}\right) \simeq \operatorname{Gauss}_{q^{-1}, ! *},
$$

up to replacing exp by its inverse.

5.5. The Whittaker category. In the next few subsections we will explain an alternative (in a sense more conceptual) construction of the objects (5.15), using some material from GLys2.

The contents of Sects. 5.5.5.7 will not be used in the sequel.

5.5.1. Since the gerbe $\mathcal{G}_{q}^{G}$ is $\mathfrak{L}(N)_{\text {Conf }}^{\omega^{\rho}}$-equivariant, it makes sense to consider the category

$$
\text { Whit }_{q, \operatorname{Conf}}(G):=\operatorname{Shv}_{\mathcal{G}_{q}^{G}}\left(\operatorname{Gr}_{G, \text { Conf }}^{\omega^{\rho}}\right)^{\mathfrak{L}(N)_{\text {Conf }}^{\omega^{\rho}}, \chi^{*}(\exp )},
$$

see GLys2, Sect, 8.4.2].

In the above formula, the superscript $\mathfrak{L}(N)_{\text {Conf }}^{\omega^{\rho}}, \chi^{*}(\exp )$ means twisted-equivariance with respect to $\mathfrak{L}(N)_{\text {Conf }}^{\omega^{\rho}}$ against the pullback of exp by means of $\chi$.

5.5.2. Consider also the categories

and

$$
\text { Whit }_{q, \operatorname{Conf}}(G)^{\leq 0}:=\operatorname{Shv}_{\mathcal{G}_{q}^{G}}\left(\bar{S}_{\text {Conf }}^{0}\right)^{\mathfrak{L}(N)_{\text {Conf }}^{\omega}, \chi^{*}(\exp )},
$$

$$
\operatorname{Whit}_{q, \operatorname{Conf}}(G)^{=0}:=\operatorname{Shv}_{\mathcal{G}_{q}^{G}}\left(S_{\text {Conf }}^{0}\right)^{\mathfrak{L}(N)_{\text {Conf }}^{\omega^{\rho}}, \chi^{*}(\exp )} .
$$

We can view Whit W Conf $(G)^{\leq 0}$ as a full subcategory of Whit $_{q, \text { Conf }}(G)$ via the closed embedding

$$
\bar{S}_{\text {Conf }}^{0} \subset \operatorname{Gr}_{G, \text { Conf }}^{\omega^{\rho}} .
$$

From the identification (5.4), we obtain:

Lemma 5.5.3. Restriction along Conf $\stackrel{\mathfrak{s}}{\rightarrow} S_{\text {Conf }}^{0}$ defines an equivalence

$$
\text { Whit }_{q, \text { Conf }}(G)^{=0} \rightarrow \operatorname{Shv}(\operatorname{Conf}) .
$$

In addition, we have: 
Lemma 5.5.4. Any object of Whit $_{q, \text { Conf }}(G)^{=0}$ supported on $\bar{S}_{\text {Conf }}^{0}-S_{\text {Conf }}^{0}$ is zero.

Proof. This is a standard stabilizer calculation: for a point on $\bar{S}_{\text {Conf }}^{0}-S_{\text {Conf }}^{0}$, the character sheaf on its stabilizer, given by the restriction of exp, is non-trivial.

Corollary 5.5.5. The functor

$$
\operatorname{Whit}_{q, \text { Conf }}(G)^{\leq 0} \rightarrow \text { Whit }_{q, \text { Conf }}(G)^{=0},
$$

given by restriction, is an equivalence.

Combining Lemma 5.5.3 and Corollary 5.5.5, we obtain:

Corollary 5.5.6. Restriction along Conf $\stackrel{\mathfrak{s}}{\rightarrow} \bar{S}_{\text {Conf }}^{0}$ defines an equivalence

$$
\text { Whit }_{q, \text { Conf }}(G) \leq 0 \rightarrow \operatorname{Shv}(\text { Conf }) .
$$

5.5.7. Let

$$
\operatorname{Vac}_{q, \text { Whit }} \in \operatorname{Whit}_{q, \operatorname{Conf}}(G)^{\leq 0} \subset \operatorname{Shv}_{\mathcal{G}_{q}^{G}}\left(\bar{S}_{\text {Conf }}^{0}\right) \subset \operatorname{Shv}_{\mathcal{G}_{q}^{G}}\left(\operatorname{Gr}_{G, \text { Conf }}^{\omega^{\rho}}\right)
$$

be the object that corresponds under the equivalence of Corollary 5.5.6 to

$$
\omega_{\text {Conf }} \in \operatorname{Shv}(\text { Conf }) \text {. }
$$

It follows from Lemma 5.5.4 that the canonical map

$$
\mathbf{j}_{!}\left(\operatorname{Vac}_{q}, \text { Whit }\left.\right|_{S_{\text {Conf }}^{0}}\right) \rightarrow \mathbf{j}_{*}\left(\left.\operatorname{Vac}_{q, \text { Whit }}\right|_{S_{\text {Conf }}^{0}}\right)
$$

is an isomorphism.

5.5.8. It is immediate from the definitions that we have a canonical isomorphism

$$
\left.\operatorname{Vac}_{q, \text { Whit }}\right|_{z^{-}} \simeq \operatorname{Vac}_{q, \text { Whit }, Z},
$$

where $-\mid$ - denotes the !-restriction.

\subsection{The semi-infinite category.}

5.6.1. Set

Set also

$$
\operatorname{SI}_{q, \text { Conf }}^{-}(G):=\operatorname{Shv}_{\mathcal{G}_{q}^{\text {ratio }}}\left(\operatorname{Gr}_{G, \text { Conf }}^{\omega^{\rho}}\right)^{\mathfrak{L}\left(N^{-}\right)_{\text {Conf }}^{\omega^{\rho}}}
$$

and

$$
\mathrm{SI}_{q, \text { Conf }}^{-}(G)^{\leq 0}:=\operatorname{Shv}_{\mathcal{G}_{q}^{\text {ratio }}}\left(\bar{S}_{\text {Conf }}^{-, \operatorname{Conf}}\right)^{\mathfrak{L}\left(N^{-}\right)_{\text {Conf }}^{\omega^{\rho}}}
$$

$$
\mathrm{SI}_{q, \text { Conf }}^{-}(G)^{=0}:=\operatorname{Shv}_{\mathcal{G}_{q}^{\text {ratio }}}\left(S_{\text {Conf }}^{-, \text {Conf }}\right)^{\mathfrak{L}\left(N^{-}\right)_{\text {Conf }}^{\omega^{\rho}}}
$$

As in the case of Whit, restriction along $\mathfrak{s}^{-}$defines an equivalence

$$
\mathrm{SI}_{q, \text { Conf }}^{-}(G)^{=0} \rightarrow \operatorname{Shv}(\text { Conf }) \text {. }
$$

5.6.2. The trivialization of $\mathcal{G}_{q}^{\text {ratio }}$ over $S_{\text {Conf }}^{- \text {, Conf }}$ defines an equivalence

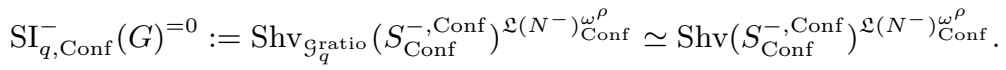

Thus, $\omega_{S_{\text {Conf }}^{-} \text {,Conf }}$ makes sense as an object of $\mathrm{SI}_{q, \text { Conf }}^{-}(G)^{=0}$; when considered in this role we will denote

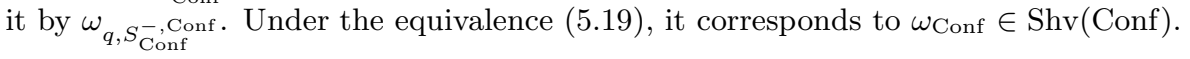

We will consider several variants of the extension of $\omega_{q, S_{\mathrm{Conf}}^{-}}$, Conf to an object of $\mathrm{SI}_{q, \text { Conf }}^{-}(G) \leq 0$.

5.6.3. One such extension is

$$
\nabla_{q}^{-}:=\left(\mathbf{j}^{-}\right)_{*}\left(\omega_{q, S_{\text {Conf }}^{-}, \text {Conf }}\right),
$$

i.e., we apply ${ }^{*}$-extension with respect to the open embedding (5.7).

Another extension is

$$
\Delta_{q}^{-}:=\left(\mathbf{j}^{-}\right) !\left(\omega_{q, S_{\text {Conf }}^{-, \text {Conf }}}\right)
$$


5.6.4. We have a canonical map

We claim:

$$
\Delta_{q}^{-} \rightarrow \nabla_{q}^{-}
$$

Proposition 5.6.5. Assume that $q$ is non-torsion valued. Then the map

$$
\Delta_{q}^{-} \rightarrow \nabla_{q}^{-}
$$

is an isomorphism.

Proof. The indscheme $\bar{S}_{\text {Conf }}^{-, \text {Conf }}$ admits a stratification parameterized by $\Lambda^{\text {pos }}$

$$
\bar{S}_{\mathrm{Conf}}^{-, \text {Conf }}=\cup_{\mu} S_{\mathrm{Conf}}^{-, \operatorname{Conf}+\mu}
$$

with

$$
S_{\text {Conf }}^{-, \text {Conf }+0}=S_{\text {Conf }}^{-, \text {Conf }}
$$

see GLys2, Sect. 12.2]. We need to show that the !-restriction of $\Delta_{q}^{-}$to any $S_{\text {Conf }}^{-, \text {Conf }+\mu}$ with $\mu \neq 0$ vanishes. We will obtain the required vanishing from considerations of equivariance with respect to

$$
\mathfrak{L}(N)_{\text {Conf }}^{\omega^{\rho}} \cdot \mathfrak{L}^{+}(T)_{\text {Conf }}:=\mathfrak{L}(B)_{\text {Conf }}^{\omega^{\rho}} \underset{\mathfrak{L}(T)_{\text {Conf }}}{\times} \mathfrak{L}^{+}(T)_{\text {Conf }}
$$

acting on $\operatorname{Gr}_{G \text {,Conf }}^{\omega}$.

The initial observation is that the gerbe $\mathcal{G}_{q}^{G}$ is equivariant with respect to $\mathfrak{L}^{+}(T)_{\text {Conf }} \cdot \mathfrak{L}(N)_{\text {Conf }}^{\omega^{\rho}}$.

The action of $\mathfrak{L}^{+}(T)_{\text {Conf }}$ preserves the section $\mathfrak{s}^{-}$. We equip $\mathcal{G}_{q}^{\text {Conf }}$ with a structure of $\mathfrak{L}^{+}(T)_{\text {Conf }^{-}}$ equivariance so that the identification

$$
\left(\mathfrak{s}^{-}\right)^{*}\left(\mathcal{G}_{q}^{G}\right) \simeq \mathcal{G}_{q}^{\mathrm{Conf}}
$$

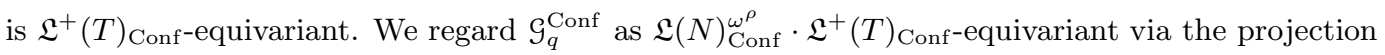

$$
\mathfrak{L}(N)_{\text {Conf }}^{\omega^{\rho}} \cdot \mathfrak{L}^{+}(T)_{\text {Conf }} \rightarrow \mathfrak{L}^{+}(T)_{\text {Conf }} .
$$

This equips the gerbe $\mathcal{G}_{q}^{\text {ratio }}$ also with a $\mathfrak{L}(N)_{\text {Conf }}^{\omega^{\rho}} \cdot \mathfrak{L}^{+}(T)_{\text {Conf-equivariant structure. }}$

With respect to the above equivariance structure on $\mathcal{G}_{q}^{\text {ratio }}$, the object $\omega_{q, S_{\text {Conf }}^{-} \text {, Conf }}$ is equivariant. Hence, so is $\Delta_{q}^{-}$. Therefore, so is its !-restriction to any $S_{\mathrm{Conf}}^{-, \operatorname{Conf}+\mu}$.

However, we claim that if $q$ is non-torsion valued, and $\mu \neq 0$, the category

$$
\operatorname{Shv}_{\mathcal{G}_{q}^{\text {ratio }}}\left(S_{\text {Conf }}^{-, \operatorname{Conf}+\mu}\right)^{\mathfrak{L}(N)_{\text {Conf }}^{\omega} \cdot \mathfrak{L}^{+}(T)_{\text {Conf }}}
$$

is zero.

Indeed, we claim that for any point on this stratum, the character sheaf on the stabilizer of this

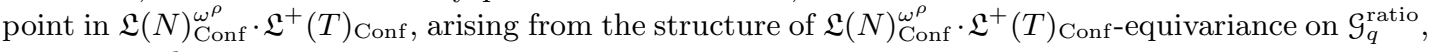
is non-trivial.

Indeed, the point in question lives over a point

$$
D=\Sigma \lambda_{k} \cdot x_{k} \in \text { Conf }
$$

and it belongs to

$$
\prod_{k} S_{x_{k}}^{-, \lambda_{k}+\mu_{k}} \subset \prod_{k} \operatorname{Gr}_{G, x_{k}}^{\omega^{\rho}}=\operatorname{Gr}_{G, \mathrm{Conf}}^{\omega^{\rho}} \underset{\mathrm{Conf}}{\times}\{D\}, \quad \Sigma \mu_{k}=\mu .
$$

Translating by means of

$$
\prod_{k} \mathfrak{L}(N)_{x_{k}}^{\omega^{\rho}} \simeq \mathfrak{L}(N)_{\text {Conf }}^{\omega^{\rho}} \underset{\text { Conf }}{\times}\{D\},
$$

we can assume that our point is invariant with respect to

$$
\prod_{k} \mathfrak{L}^{+}(T)_{x_{k}} \simeq \mathfrak{L}^{+}(T)_{\text {Conf }} \underset{\text { Conf }}{\times}\{D\} .
$$


Now, the character sheaf on (5.20) arising from the structure of $\mathfrak{L}^{+}(T)_{\text {Conf-equivariance on } \mathcal{G}_{q}^{\text {ratio }}}$ equals the pullback of

$$
\underset{k}{\bigotimes} \Psi_{b\left(\mu_{k},-\right)}
$$

along

$$
\prod_{k} \mathfrak{L}^{+}(T)_{x_{k}} \rightarrow \prod_{k} T
$$

where $\Psi$ denotes the Kummer local system.

This character sheaf is non-trivial, since for $\mu^{\prime} \neq 0$, the element

$$
b\left(\mu^{\prime},-\right) \in \operatorname{Hom}(\Lambda, \mathfrak{Z})
$$

is non-zero, by the assumption on $q$.

5.6.6. Finally, we introduce the third object of $\mathrm{SI}_{q, \text { Conf }}^{-}(G)^{\leq 0}$ that we will consider, to be denoted $\mathrm{IC}_{q}^{\frac{\infty}{2},-}$.

Breaking $G$ into simple factors, we can assume that $q$ is either torsion-valued or non-torsion valued.

When $q$ is non-torsion valued, we set

$$
\Delta_{q}^{-}=: \mathrm{IC}_{q}^{\frac{\infty}{2},-}:=\nabla_{q}^{-},
$$

where the composite isomorphism is justified by Proposition 5.6 .5

When $q$ is torsion-valued, we let $\mathrm{IC}_{q}^{\frac{\infty}{2},-}$ be the object introduced in GLys2, Sect. 18.3.2] under the name "metaplectic semi-infinite IC sheaf", and denoted there by $\mathrm{IC}_{q, \text { Conf }}^{\frac{\infty}{2},-}$

Remark 5.6.7. Here are three ways that describe the nature of $\mathrm{IC}_{q, \mathrm{Conf}}^{\frac{\infty}{2},-}$ :

(i) We have a naturally defined projection $p: \bar{S}_{\text {Conf }}^{-, \text {Conf }} \rightarrow \overline{\operatorname{Bun}}_{B^{-}}$, and the gerbe $\mathcal{G}_{q}^{\text {ratio identifies with }}$ the pullback of the same-named gerbe on $\overline{B u n}_{B^{-}}$, where the latter is the ratio of the pullbacks of the corresponding gerbes along the projections

$$
\operatorname{Bun}_{G} \leftarrow \overline{\operatorname{Bun}}_{B^{-}} \rightarrow \operatorname{Bun}_{T}
$$

respectively. The gerbe $\mathcal{G}_{q}^{\text {ratio }}$ on $\overline{\mathrm{Bun}}_{B^{-}}$is canoniocally trivialized over $\mathrm{Bun}_{B^{-}}$, and let $\mathrm{IC}_{q, \text { glob }}^{\frac{\infty}{2},-}$ define the Goresky-MacPherson extension of the (shifted) constant sheaf on $\overline{\mathrm{Bun}}_{B^{-}}$, viewed as a $\mathcal{G}_{q}^{\text {ratio }}$-twisted perverse sheaf. Then we have

$$
\mathrm{IC}_{q, \text { Conf }}^{\frac{\infty}{2},-} \simeq p^{!}\left(\mathrm{IC}_{q, \text { glob }}^{\frac{\infty}{2},-}\right)
$$

The above isomorphism describes $\mathrm{IC}_{q, \text { Conf }}^{\frac{\infty}{2},-}$ uniquely. However, its one disadvantage is that it is non-local on $X$ (since $\overline{\mathrm{Bun}}_{B^{-}}$is a global object). In particular, from this definition, the factorization property of $\mathrm{IC}_{q, \text { Conf }}^{\frac{\infty}{2},-}$ is not easy to obtain.

(ii) One can introduce a Ran space version of the semi-infinite category, to be denoted $\mathrm{SI}_{q, \text { Ran }}^{-}(G)$, and equip it with a t-structure, and define an object $\mathrm{IC}_{q, \text { Ran }}^{\frac{\infty}{2},-}$ as the minimal extension of the dualizing sheaf on $S_{\text {Ran }}^{-, \text {Conf }}$. The object $\mathrm{IC}_{q, \text { Conf }}^{\frac{\infty}{2},-}$ can be obtained from $\mathrm{SI}_{q, \operatorname{Ran}}^{-}(G)$ by restriction.

(iii) One can define $\mathrm{IC}_{q, \text { Conf }}^{\frac{\infty}{2},-}$ by an explicit colimit procedure, mimicking [Ga1, Sect. 2.3]. 
5.6.8. Note that by construction, we have an isomorphism

$$
\left.\mathrm{IC}_{q, z} \simeq \omega_{q, S_{\text {Conf }}^{-, \text {Conf }}}\right|_{\mathcal{Z}}[-\mathrm{deg}]
$$

as objects of $\operatorname{Shv}_{\mathcal{G}_{q} \text { Conf }}(\stackrel{\circ}{\mathcal{z}})$, where we remind that $-\left.\right|_{-}$stands for !-restriction.

The isomorphism extends to an isomorphism

$$
\left.\nabla_{q, Z}^{-} \simeq \nabla_{q}^{-}\right|_{z^{-}}[\operatorname{deg}]
$$

as objects of $\operatorname{Shv}_{\mathcal{G}_{q}^{\text {Conf }}}\left(Z^{-}\right)$(indeed, both sides are ${ }^{*}$-extensions from $\stackrel{\circ}{Z}$ ).

In addition, we claim:

Proposition 5.6.9. The isomorphism (5.21) extends to isomorphisms

$$
\begin{gathered}
\left.\Delta_{q, Z}^{-} \simeq \Delta_{q}^{-}\right|_{z^{-}}[-\mathrm{deg}] \\
\left.\mathrm{IC}_{q, z^{-}} \simeq \mathrm{IC}_{q^{\frac{\infty}{2}},-}\right|_{z^{-}}[-\mathrm{deg}]
\end{gathered}
$$

as objects of $\operatorname{Shv}_{\mathcal{G}_{q}^{\text {Conf }}}\left(Z^{-}\right)$.

Proof. When $q$ is non-torsion-valued, the assertion follows from Propositions 5.4.4 and 5.6.5.

Assume now that $q$ is torsion-valued. In this case, the isomorphism (5.23) is a metaplectic version of the combination of Ga1, Theorem 3.2.4] and Ga2, Corollary 3.6.5]. The isomorphism (5.24) is a metaplectic version of [Ga2, Proposition 3.8.3].

\subsection{Gauss objects via the Whittaker and the semi-infinite category.}

5.7.1. Consider the objects

$$
\operatorname{Vac}_{q}, \text { Whit } \stackrel{!}{\otimes} \nabla_{q}^{-}, \quad \operatorname{Vac}_{q}, \text { Whit } \stackrel{!}{\otimes} \Delta_{q}^{-} \text {and } \operatorname{Vac}_{q, \text { Whit }} \stackrel{!}{\otimes} \mathrm{IC}_{q}^{\frac{\infty}{2},-}
$$

in the category

$$
\operatorname{Shv}_{\mathcal{G}_{q}^{\text {Conf }}}\left(\operatorname{Gr}_{G, \text { Conf }}^{\omega^{\rho}}\right)
$$

By construction, they are supported on

$$
\bar{z} \subset \operatorname{Gr}_{G, \text { Conf }}^{\omega^{\rho}}
$$

so we can think of them as objects of $\operatorname{Shv}_{\mathcal{G}_{q} \text { Conf }}(\bar{z})$.

Furthermore, it follows from the definitions that that their further !-restrictions to $\stackrel{\circ}{z} \subset \bar{z}$ all identify canonically with

We claim:

$$
\left.\left.\left.\operatorname{Gauss}_{q, *}\right|_{\check{Z}} \simeq \operatorname{Gauss}_{q, !}\right|_{\mathcal{Z}} \simeq \operatorname{Gauss}_{q, ! *}\right|_{\check{Z}}
$$

Proposition 5.7.2. The above identifications extend to identifications

over all of $\bar{z}$.

$$
\begin{gathered}
\operatorname{Vac}_{q, \text { Whit }} \stackrel{!}{\otimes} \nabla_{q}^{-} \simeq \operatorname{Gauss}_{q, *}, \quad \operatorname{Vac}_{q, \text { Whit }} \stackrel{!}{\otimes} \Delta_{q}^{-} \simeq \operatorname{Gauss}_{q, !}, \\
\operatorname{Vac}_{q, \text { Whit }} \stackrel{!}{\otimes} \mathrm{IC}_{q}^{\frac{\infty}{2},-} \simeq \operatorname{Gauss}_{q, ! *}
\end{gathered}
$$

Proof. By the isomorphism (5.17), the objects in the left-hand side are ${ }^{*}$-extensions of their respective restrictions to $z^{-}$. By Theorem 5.4.7 the same is true for the objects appearing in the right-hand side. Hence, it is enough to establish the corresponding isomorphisms over $z^{-}$. Now the assertion follows from the isomorphisms (5.22), (5.23) and (5.24), respectively, 


\section{Factorization algebras arising from the Whittaker sheaf}

In this section we will state and prove the main results of this paper: they assert that the factorization algebras $\Omega_{q}$, introduced earlier, can be obtained geometrically from sheaves on the Zastava space.

\subsection{Factorization algebras $\Omega_{q, \mathrm{Whit}}^{\mathrm{sml}}, \Omega_{q, \mathrm{Whit}}^{\mathrm{Lus}}$ and $\Omega_{q, \mathrm{Whit}}^{\mathrm{DK}}$.}

6.1.1. We define the objects

$$
\begin{gathered}
\Omega_{q, \text { Whit }}^{\mathrm{DK}}, \Omega_{q, \text { Whit }}^{\mathrm{Lus}} \text { and } \Omega_{q, \mathrm{Whit}}^{\mathrm{sml}} \\
\bar{\pi}_{!}\left(\operatorname{Gauss}_{q, *}\right), \bar{\pi}_{!}\left(\operatorname{Gauss}_{q, !}\right) \text { and } \bar{\pi}_{!}\left(\operatorname{Gauss}_{q, ! *}\right)
\end{gathered}
$$$$
\text { in } \operatorname{Shv}_{\mathcal{G}_{q}^{\text {Conf }}}(\text { Conf }) \text { to be }
$$

respectively.

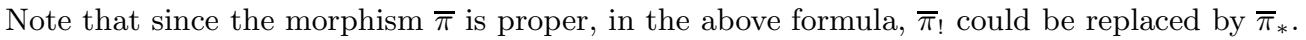

Remark 6.1.2. The interpretation of the Gauss objects given in Sect. 5.7 shows that we can construct $\Omega_{q, \text { Whit }}^{?}$ as a value on $\mathrm{Vac}_{q}$, Whit of a Jacquet-type functor

$$
J_{\text {Whit }}^{?}: \operatorname{Whit}_{q, \text { Conf }}(G) \rightarrow \operatorname{Shv}_{\mathcal{G}_{q}^{\text {Conf }}}(\text { Conf })
$$

defined by

$$
J_{\text {Whit }}^{\mathrm{DK}}(\mathcal{F}):=\bar{\pi}_{!}\left(\mathcal{F} \stackrel{!}{\otimes} \Delta_{q}^{-}\right), \quad J_{\text {Whit }}^{\mathrm{Lus}}(\mathcal{F}):=\bar{\pi}_{!}\left(\mathcal{F} \stackrel{!}{\otimes} \nabla_{q}^{-}\right)
$$

and

respectively.

$$
J_{\text {Whit }}^{\mathrm{sml}}(\mathcal{F}):=\bar{\pi}_{!}\left(\mathcal{F} \stackrel{!}{\otimes} \mathrm{IC}_{q}^{\frac{\infty}{2},-}\right),
$$

6.1.3. The factorization structure on the objects (5.15) gives rise to a structure of factorization algebra in $\operatorname{Shv}_{\mathcal{G}_{q}^{\text {Conf }}}($ Conf) on the objects (6.1).

6.1.4. From Corollary 5.4 .9 we obtain:

Corollary 6.1.5. We have the isomorphisms

$$
\mathbb{D}^{\text {Verdier }}\left(\Omega_{q, \text { Whit }}^{\text {DK }}\right) \simeq \Omega_{q^{-1}, \text { Whit }}^{\text {Lus }} \text { and } \mathbb{D}^{\text {Verdier }}\left(\Omega_{q, \text { Whit }}^{\text {sml }}\right) \simeq \Omega_{q^{-1}, \text { Whit }}^{\text {sml }}
$$

Proof. We only have to show that replacing exp by its inverse does not affect the objects (6.1).

The above replacement can be affected by the action of $(-1) \in \mathbb{G}_{m}$ as an automorphism of $\mathbb{G}_{a}$. The morphism $\chi: \bar{z} \rightarrow \mathbb{G}_{a}$ is $\mathbb{G}_{m}$-equivariant with respect to the action of $\mathbb{G}_{m}$ on $\bar{z}$ given by

$$
\mathbb{G}_{m} \stackrel{2 \rho}{\rightarrow} T \subset \mathfrak{L}^{+}(T)
$$

and the action of the latter on $\operatorname{Gr}_{G}^{\omega^{\rho}}$, and the square of the action of $\mathbb{G}_{m}$ on $\mathbb{G}_{a}$ by dilations.

The result now follows from the fact that the morphism $\bar{\pi}$ is $\mathbb{G}_{m}$-equivariant.

6.1.6. We now claim:

Proposition 6.1.7. The objects (6.1) are perverse.

Proof. Recall that $\pi^{-}$denotes the restriction of $\pi$ to $z^{-}$. Recall also that the morphism $\pi^{-}$is affine, while

$$
\operatorname{Gauss}_{q, ?}^{-}=\left.\operatorname{Gauss}_{q, ?}\right|_{z^{-}}
$$

are perverse for $?=*$, ! or $! *$. Hence $\pi_{!}^{-}\left(\operatorname{Gauss}_{q, ?}^{-}\right)$is cohomologically $\geq 0$, while $\pi_{*}^{-}\left(\right.$Gauss $\left._{q, ?}^{-}\right)$is cohomologically $\leq 0$.

However, by Theorem 5.4.7 we have

$$
\pi_{!}^{-}\left(\operatorname{Gauss}_{q, ?}^{-}\right) \simeq \Omega_{q, \text { Whit }}^{?} \simeq \pi_{*}^{-}\left(\operatorname{Gauss}_{q, ?}^{-}\right)
$$

for each of the three versions. 


\subsection{Statement of the main results.}

6.2.1. First, we claim:

Proposition 6.2.2. Assume that $q$ is non-degenerate. Then the natural maps

$$
\Omega_{q, \text { Whit }}^{\text {Lus }} \rightarrow \Omega_{q, \text { Whit }}^{\text {sml }} \rightarrow \Omega_{q, \text { Whit }}^{\mathrm{DK}}
$$

become isomorphisms after restriction to Conf. The resulting perverse sheaf on $\mathrm{C}^{\circ}$. $\stackrel{\circ}{\Omega}$.

Proof. By factorization, the statement reduces to showing that for every simple coroot, the restriction of the objects (6.1) to Conf $^{-\alpha_{i}}$ identifies with $\mathrm{e}_{X}[1]$. We have:

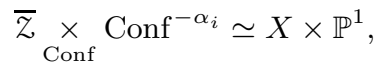

while

$$
\stackrel{\circ}{z} \underset{\text { Conf }}{\times} \operatorname{Conf}^{-\alpha_{i}} \subset z^{-} \underset{\text { Conf }}{\times} \operatorname{Conf}^{-\alpha_{i}} \subset \bar{z} \underset{\text { Conf }}{\times} \operatorname{Conf}^{-\alpha_{i}}
$$

correspond under the identification (6.2)

$$
X \times \mathbb{G}_{m} \subset X \times \mathbb{A}^{1} \subset X \times \mathbb{P}^{1},
$$

respectively.

Now, it is shown in GLys2, Sect. 18.4.10] that in terms of the identification (6.2) (and the trivialization of the gerbe $\mathcal{G}_{q}^{\mathrm{Conf}}$ over $\operatorname{Conf}^{-\alpha_{i}}$ ), we have:

$$
\left.\operatorname{Gauss}_{q, ?}^{-}\right|_{\mathcal{Z}} \simeq \mathrm{e}_{X}[1] \otimes \operatorname{Gauss}_{q\left(\alpha_{i}\right)}[1]
$$

where for $\zeta \in \mathfrak{Z}$ we denote

$$
\operatorname{Gauss}_{\zeta}:=\left.\exp \right|_{\mathbb{G}_{m}} \stackrel{*}{\otimes} \Psi_{\zeta},
$$

where we recall that $\Psi_{\zeta}$ denotes the Kummer local system on $\mathbb{G}_{m}$ corresponding to the element $\zeta \in \mathfrak{Z}$.

The objects

$$
\operatorname{Gauss}_{q, *}^{-}, \operatorname{Gauss}_{q, !}^{-} \operatorname{Gauss}_{q, ! *}^{-}
$$

are given by $*_{-}$, !- and !*- extensions of $\left.\operatorname{Gauss}_{q, ?}^{-}\right|_{\odot}$, respectively. However, for $q\left(\alpha_{i}\right) \neq 0$ (the condition that $q$ is non-degenerate), the extension of $\Psi_{q\left(\alpha_{i}\right)}$ along $\mathbb{G}_{m} \hookrightarrow \mathbb{A}^{1}$ is clean.

Finally, we have:

$$
H^{i}\left(\mathbb{G}_{m}, \operatorname{Gauss}_{\zeta}\right)=\left\{\begin{array}{l}
0 \text { for } i \neq 1 \\
\text { e for } i=1
\end{array}\right.
$$

Remark 6.2.3. The name Gauss stems from the fact that the right-hand side in (6.3) is the local system, whose cohomology is the geometric counterpart of the Gauss sum.

6.2.4. We are now ready to state the two main results of this paper.

Theorem 6.2.5. Assume that $q$ avoids small torsion 17 . Then the identification of Proposition 6.2.2 extends to isomorphisms

$$
\Omega_{q, \text { Whit }}^{\mathrm{DK}} \simeq \Omega_{q}^{\mathrm{DK}} \text { and } \Omega_{q, \mathrm{Whit}}^{\mathrm{Lus}} \simeq \Omega_{q}^{\mathrm{Lus}} .
$$

Note that the two isomorphisms stated in Theorem 6.2.5 are obtained from one another by Verdier duality.

Theorem 6.2.6. Assume that $q$ avoids small torsion. Then the identification of Proposition 6.2.2 extends to an isomorphism

$$
\Omega_{q, \mathrm{Whit}}^{\mathrm{sml}} \simeq \Omega_{q}^{\mathrm{sml}}
$$

\footnotetext{
${ }^{17}$ See Sect. 1.1 .5 for what this means.
} 
Remark 6.2.7. It is shown in $\left[\mathrm{Ras}\right.$, Theorem 3.4.1] that for $q=0$, the object $\Omega_{q, \text { Whit }}^{\mathrm{sml}}$ vanishes. It is then a formal consequence of Theorem 6.6.2 below that in this case $\Omega_{q, \text { Whit }}^{\text {Lus }} \simeq \Omega^{\mathrm{cl}}$.

6.2.8. The plan of the rest of the paper is the following: in the next subsection we will show that Theorem 6.2.6 is equivalent to Theorem 6.3.8 which asserts a certain sub-top cohomology vanishing. In Sect. 6.5 we will show that Theorems 6.2.5 and 6.2.6 are equivalent to one another.

In Sect. 6.6 we will prove Theorems 6.2.5 and 6.2.6 over a ground field of characteristic 0, thereby also proving Theorem 6.3.8.

Finally, in Sect. 7 we will show that the assertion of Theorem 6.3.8 over a ground field of characteristic 0 implies the assertion of Theorem 6.3.8 over any ground field. This will complete the proof of Theorems 6.2 .5 and 6.2.6 over any ground field as well.

6.3. Interpretation as sub-top cohomology vanishing. The goal of this subsection is to reduce the assertion of Theorem 6.2.6 to an essentially combinatorial statement about MV cycles.

6.3.1. Note that by Corollary 6.1.5 induction on $|\lambda|$, étale invariance and factorization, the assertion of Theorem 6.2.6 is equivalent to the fact that

$$
\left(\iota_{\lambda}\right)^{!}\left(\Omega_{q, \mathrm{Whit}}^{\mathrm{sml}}\right) \in \operatorname{Vect}_{\mathcal{G}_{q, x}^{\lambda}}
$$

lives in cohomological degrees $\geq 2$, as long as $\lambda$ is not a negative simple coroot.

Realizing $\Omega_{q, \text { Whit }}^{\mathrm{sml}}$ as

by base change, we have

$$
\pi_{*}^{-}\left(\operatorname{Gauss}_{q, ! *}^{-}\right)
$$

$$
\left(\iota_{\lambda}\right)^{!}\left(\Omega_{q, \mathrm{Whit}}^{\mathrm{sml}}\right) \simeq \mathrm{C} \cdot\left(S^{0} \cap \bar{S}^{-, \lambda},\left.\mathrm{IC}_{q, Z}^{-}\right|_{S^{0} \cap \bar{S}^{-, \lambda}} \stackrel{*}{\otimes} \chi^{*}(\exp )\right) .
$$

Here $S^{0}$ (resp., $\bar{S}^{-, \lambda}$ ) are the fibers of $S_{\text {Conf }}^{0}$ (resp., $S_{\text {Conf }}^{-, \text {Conf }}$ ) over the point $\lambda \cdot x \in$ Conf. In other words, they are the 0 -th semi-infinite orbit (resp., closure of the $\lambda$ negative semi-infinite orbit).

Thus, the assertion of Theorem 6.2 .6 is equivalent to the fact that the cohomology

$$
H^{i}\left(S^{0} \cap \bar{S}^{-, \lambda},\left.\mathrm{IC}_{q, Z}^{-}\right|_{S^{0} \cap \bar{S}^{-, \lambda}} \stackrel{*}{\otimes} \chi^{*}(\exp )\right)
$$

vanishes for $i=1$ as long as $\lambda$ is not a negative simple coroot.

We note also that the cohomology (6.4) vanishes for $i=0$ (for all $\lambda \in \Lambda^{\text {neg }}-0$, including the negative simple coroots). This expresses the fact that $\Omega_{q, \text { Whit }}^{\mathrm{sml}}$ lives in perverse degrees $\geq 0$.

Remark 6.3.2. For future reference, we note that we also have

$$
\begin{aligned}
\left(\iota_{\lambda}\right) !\left(\Omega_{q, \text { Whit }}^{\mathrm{DK}}\right) \simeq \mathrm{C}\left(S^{0} \cap S^{-, \lambda},\left.\mathrm{IC}_{q, \grave{z}}\right|_{S^{0} \cap S^{-, \lambda}} \stackrel{*}{\otimes} \chi^{*}(\exp )\right) & \simeq \\
& \simeq \mathrm{C}\left(S^{0} \cap S^{-, \lambda}, \omega_{S^{0} \cap S^{-, \lambda}}[\langle\lambda, 2 \check{\rho}\rangle] \stackrel{*}{\otimes} \chi^{*}(\exp )\right) .
\end{aligned}
$$

Consider the individual cohomologies.

$$
H^{i}\left(S^{0} \cap S^{-, \lambda^{\prime}}, \omega_{S^{0} \cap S^{-, \lambda^{\prime}}}\left[\left\langle\lambda^{\prime}, 2 \check{\rho}\right\rangle\right] \stackrel{\otimes}{*}^{*}(\exp )\right)
$$

The expression (6.5) vanishes for $i=0$; indeed, this is equivalent to the fact that $\Omega_{q, \text { Whit }}^{\mathrm{DK}}$ lives in perverse degrees $\geq 0$. However, we will shortly see a direct analysis proving this vanishing (this will be a rather simple cohomological estimate), see Sects. 7.2.4 7.2.5.

The vanishing of (6.5) in degree $i=1$ is equivalent to the fact that the map

$$
\Omega_{q, \text { Whit }}^{\mathrm{DK}} \rightarrow H^{0}\left(\left(\jmath^{\lambda}\right)_{*} \circ\left(\jmath^{\lambda}\right)^{*}\left(\Omega_{q, \text { Whit }}^{\mathrm{DK}}\right)\right)
$$

is an injection of perverse sheaves. As we will see in Sect. 6.5 this is essentially equivalent to the assertion of Theorem 6.2.5. In Sect. 6.3.5 we will see that this vanishing is equivalent also to the assertion of Theorem 6.2.6 
Thus, we will eventually prove that the cohomology (6.5) vanishes also in degree $i=1$ (provided that $q$ avoids small torsion). But this will be a much subtler analysis.

6.3.3. First, we claim:

Lemma 6.3.4. The restriction map along $S^{0} \cap S^{-, \lambda} \hookrightarrow S^{0} \cap \bar{S}^{-, \lambda}$

$$
\begin{aligned}
& H^{i}\left(S^{0} \cap \bar{S}^{-, \lambda},\left.\mathrm{IC}_{q, Z}^{-}\right|_{S^{0} \cap \bar{S}^{-, \lambda}} \stackrel{*}{\otimes} \chi^{*}(\exp )\right) \rightarrow \\
& \quad \rightarrow H^{i}\left(S^{0} \cap S^{-, \lambda},\left.\mathrm{IC}_{q, \stackrel{\circ}{z}}\right|_{S^{0} \cap S^{-, \lambda}} \stackrel{*}{\otimes} \chi^{*}(\exp )\right) \simeq H^{i}\left(S^{0} \cap S^{-, \lambda}, \omega_{S^{0} \cap S^{-, \lambda}}[\langle\lambda, 2 \check{\rho}\rangle] \stackrel{*}{\otimes} \chi^{*}(\exp )\right)
\end{aligned}
$$

induces an isomorphism in degree $i=1$.

Proof. Writing $\bar{S}^{-, \lambda}-S^{-, \lambda}$ as the union of $S^{-, \lambda^{\prime}}$ with $\lambda^{\prime}<\lambda$, by the Cousin spectral sequence, it suffices to show that

$$
H^{i}\left(S^{0} \cap S^{-, \lambda^{\prime}},\left.\left(\left.\mathrm{IC}_{q, Z}^{-}\right|_{S^{0} \cap \bar{S}^{-, \lambda}}\right)\right|_{S^{0} \cap S^{-, \lambda^{\prime}}} \stackrel{*}{\otimes} \chi^{*}(\exp )\right)
$$

vanishes for $i=1$ and $i=2$ and $\lambda^{\prime} \neq \lambda$.

First, it follows from Remark 6.7.5 that

$$
\left.\left(\left.\mathrm{IC}_{q, Z}^{-}\right|_{S^{0} \cap \bar{S}^{-, \lambda}}\right)\right|_{S^{0} \cap S^{-, \lambda}}
$$

has the form

$$
\omega_{S^{0} \cap S^{-, \lambda^{\prime}}}\left[\left\langle\lambda^{\prime}, 2 \check{\rho}\right\rangle\right] \otimes \mathcal{E},
$$

where $\mathcal{E} \in$ Vect lives in cohomological degrees $\geq 2$.

So it suffices to show that

$$
H^{i}\left(S^{0} \cap S^{-, \lambda^{\prime}}, \omega_{S^{0} \cap S^{-, \lambda^{\prime}}}\left[\left\langle\lambda^{\prime}, 2 \check{\rho}\right\rangle\right] \stackrel{*}{\otimes}^{*}(\exp )\right)
$$

vanishes for $i \leq 0$. However, this vanishing is the expression of the fact that $\Omega_{q, \text { Whit }}^{\mathrm{DK}}$ is $\geq 0$ in the perverse t-structure, see Remark 6.3.2.

6.3.5. Thus, the assertion of Theorem 6.2.6 is equivalent to the vanishing of

$$
H^{i}\left(S^{0} \cap S^{-, \lambda}, \omega_{S^{0} \cap S^{-, \lambda}}[\langle\lambda, 2 \check{\rho}\rangle] \otimes^{*} \chi^{*}(\exp )\right) \in \operatorname{Vect}_{\mathcal{G}_{q, x}^{\lambda}}
$$

for $i=1$ when $\lambda$ is not a negative simple coroot. (Along the way we will also see that it vanishes for $i=0$ for all $\lambda \in \Lambda^{\text {neg }}-0$ for a much simpler reason.)

In formula (6.6) we view the ${ }^{*}$-restriction of $\chi^{*}(\exp )$ to $S^{0} \cap S^{-, \lambda}$ as a $\mathcal{G}_{q, x^{-}}^{\lambda}$-twisted sheaf via the identifications

$$
\left.\left.\left.\left(\mathcal{G}_{q}^{G}\right)_{0}\right|_{S^{0} \cap S^{-, \lambda}} \simeq \mathcal{G}_{q}^{G}\right|_{S^{0} \cap S^{-, \lambda}} \simeq \mathcal{G}_{q}^{\lambda}\right|_{S^{0} \cap S^{-, \lambda}},
$$

where:

- $\left(\mathcal{G}_{q}^{G}\right)_{0}$ is the trivial gerbe;

- The identification $\left.\left.\left(\mathcal{G}_{q}^{G}\right)_{0}\right|_{S^{0} \cap S^{-, \lambda}} \simeq \mathcal{G}_{q}^{G}\right|_{S^{0} \cap S^{-, \lambda}}$ comes by restriction along $S^{0} \cap S^{-, \lambda} \rightarrow S^{0}$ from the trivialization of $\left.\mathcal{G}_{q}^{G}\right|_{S^{0}}$;

- The identification $\left.\left.\mathcal{G}_{q}^{G}\right|_{S^{0} \cap S^{-, \lambda}} \simeq \mathcal{G}_{q}^{\lambda}\right|_{S^{0} \cap S^{-, \lambda}}$ comes by restriction along $S^{0} \cap S^{-, \lambda} \rightarrow S^{-, \lambda}$ from the identification $\left.\left.\mathcal{G}_{q}^{G}\right|_{S^{-, \lambda}} \simeq \mathcal{G}_{q}^{\lambda}\right|_{S^{-, \lambda}}$. 
6.3.6. Let us choose a trivialization of the fiber of $\mathcal{G}_{q, x}^{\lambda}$ of $\mathcal{G}_{q}^{\lambda}$ at $x \in X$. We obtain that (6.7) gives rise to a local system on $S^{0} \cap S^{-, \lambda}$, to be denoted $\Psi_{q, \lambda}$, which is well-defined up to a $\mathrm{e}^{\times}$-torsor. Moreover, $\Psi_{q, \lambda}$ is twisted $T$-equivariant against a Kummer sheaf on $T$ corresponding to the homomorphism

$$
\Lambda \rightarrow \mathfrak{Z}, \quad \mu \mapsto b(\lambda, \mu) .
$$

We will give a more explicit description of $\Psi_{q, \lambda}$ in Sect. 7.4.4.

We obtain that (6.6), shifted cohomologically by $-[\langle\lambda, 2 \check{\rho}\rangle]$, viewed as a plain vector space (due to the chosen trivialization of $\mathcal{G}_{q, x}^{\lambda}$ ), identifies with

$$
H^{i}\left(S^{0} \cap S^{-, \lambda}, \omega_{S^{0} \cap S^{-, \lambda}} \stackrel{*}{\otimes} \Psi_{q, \lambda} \stackrel{*}{\otimes} \chi^{*}(\exp )\right) .
$$

Thus, Theorem 6.2.6 is equivalent to the vanishing of (6.9) in degree

$$
i=1+\langle\lambda, 2 \check{\rho}\rangle
$$

(and also $i=\langle\lambda, 2 \check{\rho}\rangle$ ).

6.3.7. Up to replacing exp by its inverse, the cohomology in (6.9) is dual to

$$
H_{c}^{i}\left(S^{0} \cap S^{-, \lambda}, \Psi_{q, \lambda} \otimes \chi^{*}(\exp )\right) .
$$

Thus, we obtain that Theorem 6.2.6 is equivalent to the following one:

Theorem 6.3.8. For $q$ that avoids small torsion and $\lambda$ not a negative simple coroot, the cohomology (6.10) vanishes in (the sub-top) degree

$$
i=-\langle\lambda, 2 \check{\rho}\rangle-1 .
$$

We will also see that that the cohomology in (6.10) vanishes in (the top) degree $i=-\langle\lambda, 2 \check{\rho}\rangle$ for all $\lambda \in \Lambda^{\text {neg }}-0$.

\subsection{The non-torsion valued case.}

6.4.1. In this subsection we will prove the following assertion:

Theorem 6.4.2. Let $q$ be non-torsion-valued. Then:

(a) The maps

$$
\Omega_{q, \mathrm{Whit}}^{\mathrm{DK}} \rightarrow \Omega_{q, \mathrm{Whit}}^{\mathrm{sml}} \rightarrow \Omega_{q, \mathrm{Whit}}^{\mathrm{Lus}}
$$

are isomorphisms.

(b) The isomorphism $\left.\Omega_{q, \mathrm{Whit}}^{\mathrm{sml}}\right|_{\mathrm{Conf}} ^{\circ} \simeq \stackrel{\circ}{\Omega}$ extends to an isomorphism

$$
\Omega_{q, \mathrm{Whit}}^{\mathrm{sml}} \simeq \Omega_{q}^{\mathrm{sml}} .
$$

6.4.3. Note that point (a) of Theorem 6.4.2 follows from Proposition 5.4.4. So, the essence of the theorem is point (b), which is a particular case of Theorem 6.2.6.

As we have just seen, Theorem 6.2.6 is equivalent to Theorem 6.3.8. Thus, we claim that Theorem 6.3.8 holds when $q$ is non-torsion valued.

The required cohomological estimate is performed in Sect. 7.5] see Remark 7.5.10. The same calculation is also performed in Ga3, Sects. 6.3-6.5].

$\square[$ Theorem 6.4 .2

6.5. Adding the formal parameter. The goal of this subsection is to show that Theorems 6.2.5 and 6.2 .6 are logically equivalent. 
D. GAITSGORY

6.5.1. Following Sect. A.1 we can introduce a version of $\operatorname{Gauss}_{q, *}$ over e匹 $\hbar \rrbracket$, to be denoted

$$
\operatorname{Gauss}_{\llbracket \llbracket \hbar, *} \in \operatorname{Shv}_{\mathcal{G}_{q \llbracket \hbar \rrbracket}^{\operatorname{Conf}}}\left(\operatorname{Gr}_{G, \text { Conf }}^{\omega^{\rho}}\right) .
$$

Set

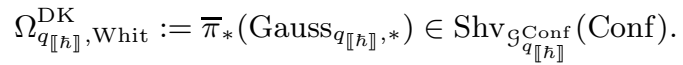

As in Proposition 6.2.2 we have:

Proposition 6.5.2. There exists a canonical isomorphism

$$
\left.\Omega_{q_{\llbracket \hbar \rrbracket}^{\mathrm{DK}}, \text { Whit }}\right|_{\mathrm{Conf}} ^{\circ} \simeq \stackrel{\circ}{\Omega}_{q_{\llbracket \hbar \rrbracket}} .
$$

6.5.3. Let

$$
\Omega_{q_{((\hbar))}, \text { Whit }}:=\bar{\pi}_{*}\left(\operatorname{Gauss}_{q_{((\hbar))}, *}\right) \in \operatorname{Shv}_{\mathcal{G}_{q_{((\hbar))}^{\text {Conf }}}}(\text { Conf })
$$

be the localization of $\Omega_{q_{\llbracket \hbar \rrbracket}}^{\mathrm{DK}}$,Whit .

As in Theorem 6.4.2 we obtain that the isomorphism

$$
\left.\Omega_{q_{((\hbar))}, \text { Whit }}\right|_{\text {Conf }} ^{\circ} \simeq \stackrel{\circ}{\Omega}_{q_{((\hbar))}},
$$

induced by the isomorphism of Proposition 6.5.2 extends to an isomorphism

$$
\Omega_{q_{((\hbar))}, \text { Whit }} \simeq \Omega_{q_{((\hbar))}} .
$$

6.5.4. By construction

$$
\Omega_{q \llbracket \hbar \rrbracket}^{\mathrm{DK}}, \text { Whit } \underset{\mathrm{e} \llbracket \hbar \rrbracket}{\otimes} \mathrm{e} \simeq \Omega_{q, \mathrm{Whit}}^{\mathrm{DK}} ;
$$

in particular, $\Omega_{q_{\llbracket \hbar \rrbracket}^{\mathrm{DK}} \text {, Whit }}^{\mathrm{D}}$ is cohomologically $\leq 0$.

From here and the isomorphism (6.12), we obtain that the isomorphism (6.11) extends uniquely to a map

$$
\Omega_{q_{\llbracket \hbar \rrbracket}^{\mathrm{DK}}, \text { Whit }}^{\mathrm{DK}} \rightarrow \Omega_{q_{\llbracket \hbar \rrbracket}^{\mathrm{DK}}}^{\mathrm{D}}
$$

We claim:

Proposition 6.5.5. For a given $q$, the following assertions are equivalent:

(i) The map (6.13) is an isomorphism;

(ii) The isomorphism of Proposition 6.2.2 extends to an isomorphism

$$
\Omega_{q, \mathrm{Whit}}^{\mathrm{DK}} \simeq \Omega_{q}^{\mathrm{DK}} .
$$

(iii) The isomorphism of Proposition 6.2.2 extends to an isomorphism

$$
\Omega_{q, \mathrm{Whit}}^{\mathrm{sml}} \simeq \Omega_{q}^{\mathrm{sml}} \text {. }
$$

Proof. Assertion (i) implies assertion (ii) by reduction modulo $\hbar$.

Before we prove the other equivalences, let us note that by Remark 6.3.2 and Lemma 6.3.4 point (iii) is equivalent to the fact that the map

$$
\Omega_{q, \text { Whit }}^{\mathrm{DK}} \rightarrow H^{0}\left(\left(\jmath^{\lambda}\right)_{*} \circ\left(\jmath^{\lambda}\right)^{*}\left(\Omega_{q, \text { Whit }}^{\mathrm{DK}}\right)\right)
$$

is an injection of perverse sheaves for any $\lambda$ that is not a negative simple coroot.

Let us now assume that (ii) holds, and let us deduce (iii). Indeed, by the above, this follows from the fact that

is an injection of perverse sheaves.

$$
\Omega_{q}^{\mathrm{DK}} \rightarrow H^{0}\left(\left(\jmath_{\lambda}\right)_{*} \circ\left(\jmath_{\lambda}\right)^{*}\left(\Omega_{q}^{\mathrm{DK}}\right)\right)
$$

Finally, let us assume (iii) and deduce (i). By induction and factorization, we can assume that

$$
\left(\jmath_{\lambda}\right)^{*}\left(\Omega_{q_{\llbracket \hbar \rrbracket} \text {,Whit }}^{\mathrm{DK}}\right) \rightarrow\left(\jmath_{\lambda}\right)^{*}\left(\Omega_{q_{\llbracket \hbar \rrbracket}^{\mathrm{DK}}}^{\mathrm{D}}\right)
$$


is an isomorphism. Furthermore, by (6.12), the map (6.13) becomes an isomorphism after inverting $\hbar$. Let $\mathcal{F}$ denote the cone of $(6.13)$. By the above, $\mathcal{F}$ is $\hbar$-torsion and concentrated in (perverse) degrees $\leq 0$. Hence if $\mathcal{F} \neq 0$, the object $\mathcal{F} \otimes$ e would have non-trivial cohomology in (perverse) degrees $<0$.

This would mean that the map $\mathrm{e} \llbracket \hbar \rrbracket$

$\Omega_{q, \text { Whit }}^{\mathrm{DK}} \rightarrow \Omega_{q}^{\mathrm{DK}}$

is not an injection of perverse sheaves. However, from the commutative diagram

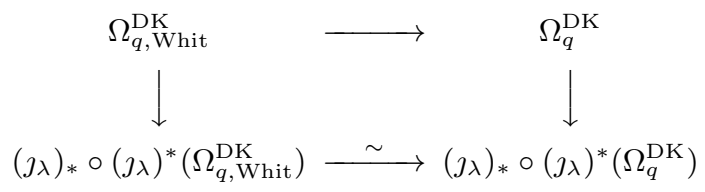

we would obtain that (6.14) is not an injection either, contradicting (iii).

6.5.6. Note that the equivalence (ii) $\Leftrightarrow$ (iii) in Proposition 6.5.5 combined with Theorem 6.4.2 implies the assertion of Theorem 2.1.5

6.6. Quantum Frobenius for $\Omega_{q \text {,Whit }}^{\text {Lus }}$. In this subsection we will state Theorem 6.6.2 and using it will deduce the assertions of Theorems 6.2.5 and 6.2.6 over a ground field of characteristic 0 .

6.6.1. Let $q$ be torsion-valued. We claim:

Theorem 6.6.2. There exists a canonically defined action of $\Omega^{\sharp, \mathrm{cl}}$ on $\Omega_{q, \mathrm{Lh}}^{\mathrm{Lus}}$ such that the map

$$
\Omega_{q, \text { Whit }}^{\text {Lus }} \rightarrow \Omega_{q, \text { Whit }}^{\text {sml }}
$$

is compatible with the trivial $\Omega^{\sharp, \mathrm{cl}}$-action on $\Omega_{q, \mathrm{Whit}}^{\mathrm{sml}}$, and gives rise to an identification

$$
\left(\left(\text { Cone }\left(\Omega^{\sharp, c l}\right)_{X} \rightarrow\left(\Omega_{q, \text { Whit }}^{\text {Lus }}\right)_{X}\right)\right)_{\left(\Omega^{\sharp, c l}\right)_{X}}^{\otimes} \omega_{X} \rightarrow \Omega_{q, \text { Whit }}^{\text {sml }}
$$

This theorem will be proved in Sect. 6.7.

Remark 6.6.3. The assertion of Theorem 6.6.2 holds for any torsion-valued $q$ (i.e., we do not need the assumption that $q$ avoid small torsion).

So, quantum Frobenius exists for $\Omega_{q \text {,Whit }}^{\mathrm{Lus}}$ for all torsion-valued $q$ (including the case when $q$ is degenerate), but for it to exist for $\Omega_{q}^{\mathrm{Lus}}$ (which we already know to be isomorphic to $\Omega_{q, \text { Quant }}^{\mathrm{Lus}}$ ) we need the assumption that $q$ avoid small torsion, see Remark 4.4.8 $\Omega_{q}^{\mathrm{Lus}}$.

This justifies the point of view that $\Omega_{q, \text { Whit }}^{\text {Lus }}$ is, in general, a more relevant object than $\Omega_{q, \text { Quant }}^{\text {Lus }} \simeq$

6.6.4. Note that once Theorems 6.2.5 and 6.2.6 are proved, the assertion of Theorem 4.4.2 would follow from that of Theorem 6.6.2 as in Sects. 4.4.9.4.4.11

6.6.5. Let us assume Theorem 6.6.2 and deduce Theorem 6.2.6 over a ground field of characteristic 0 . (This would also imply Theorem 6.2.5 by Proposition 6.5.5).

By induction and factorization, we can assume that the map

$$
\left(\jmath_{\lambda}\right)^{*}\left(\Omega_{q, \text { Whit }}^{\mathrm{sml}}\right) \rightarrow\left(\jmath_{\lambda}\right)^{*}\left(\Omega_{q}^{\mathrm{sml}}\right)
$$

is an isomorphism. We wish to show that this isomorphism extends across the main diagonal

$$
X \stackrel{\Delta_{\lambda}}{\hookrightarrow} X^{\lambda}
$$

A priori, $\left.\Omega_{q, \text { Whit }}^{\mathrm{sml}}\right|_{X^{\lambda}}$ has a 3 -step filtration

$$
0=F_{0} \subset F_{1} \subset F_{2} \subset F_{3}=\left.\Omega_{q, \text { Whit }}^{\text {sml }}\right|_{X^{\lambda}}
$$

with

$$
F_{2} /\left.F_{1} \simeq \Omega_{q}^{\mathrm{sml}}\right|_{X^{\lambda}}
$$


and $F_{1}$ and $F_{3} / F_{2}$ supported on the main diagonal. We wish to show that $F_{1}=F_{3} / F_{2}=0$. This is equivalent to showing that the (signed) Jordan-Holder contents of

$$
\Delta_{\lambda}^{!}\left(\Omega_{q, \text { Whit }}^{\mathrm{sml}}\right) \text { and } \Delta_{\lambda}^{!}\left(\Omega_{q}^{\mathrm{sml}}\right)
$$

are equal.

Recall that the map (6.13) becomes an isomorphism after inverting $\hbar$. Hence, the same is true for the map

$$
\Delta_{\lambda}^{*}\left(\Omega_{q_{\llbracket \hbar \rrbracket}}^{\mathrm{DK}}, \text { Whit }\right) \rightarrow \Delta_{\lambda}^{*}\left(\Omega_{q_{\llbracket \hbar \rrbracket}^{\mathrm{DK}}, \text { Whit }}^{\mathrm{DK}}\right) .
$$

From this it follows that the (signed) Jordan-Holder contents of

$$
\Delta_{\lambda}^{*}\left(\Omega_{q, \text { Whit }}^{\mathrm{DK}}\right) \text { and } \Delta_{\lambda}^{*}\left(\Omega_{q}^{\mathrm{DK}}\right)
$$

are equal. By Verdier duality, we obtain that the (signed) Jordan-Holder contents of

$$
\Delta_{\lambda}^{!}\left(\Omega_{q, \text { Whit }}^{\text {Lus }}\right) \text { and } \Delta_{\lambda}^{!}\left(\Omega_{q}^{\text {Lus }}\right)
$$

are equal.

This implies that the (signed) Jordan-Holder contents of

$$
\left(\left(\text { Cone }\left(\Omega^{\sharp, c l}\right)_{X} \rightarrow\left(\Omega_{q, \text { Whit }}^{\text {Lus }}\right)_{X}\right)\right) \underset{\left(\Omega^{\sharp, c l}\right)_{X}}{\otimes} \omega_{X}
$$

and

are also equal.

$$
\left(\left(\operatorname{Cone}\left(\Omega^{\sharp, c l}\right)_{X} \rightarrow\left(\Omega_{q}^{\text {Lus }}\right)_{X}\right)\right)_{\left(\Omega^{\sharp, c l}\right)_{X}}^{\otimes} \omega_{X}
$$

Applying Theorem 6.6.2 and Theorem 4.4.2(f), which has been proved over a ground field of characteristic 0 , we obtain that the Jordan-Holder contents of

$$
\Delta_{\lambda}^{!}\left(\Omega_{q, \text { Whit }}^{\text {sml }}\right) \text { and } \Delta_{\lambda}^{!}\left(\Omega_{q}^{\text {sml }}\right)
$$

are also equal, as required.

$\square[$ Theorem 6.2.5

\subsection{Geometric construction of the quantum Frobenius.}

6.7.1. Recall the maps

see (5.14).

$$
\mathbf{i}^{\lambda}: X^{\lambda} \times z^{-} \rightarrow z^{-}
$$

They combine to an action of the semi-group Conf on $z^{-}$, compatible with the projection $z^{-} \rightarrow$ Conf and the section $\mathfrak{s}$ : Conf $\rightarrow z^{-}$.

Pre-composing with Conf $\sharp \rightarrow$ Conf, we obtain an action of Conf ${ }^{\sharp}$ on $z^{-}$. This action induces a monoidal action of $\operatorname{Shv}\left(\operatorname{Conf}^{\sharp}\right)$ on $\operatorname{Shv}_{\mathcal{G}_{q}^{\text {ratio }}}\left(Z^{-}\right)$. Hence, given an algebra in $\operatorname{Shv}\left(\operatorname{Conf} f^{\sharp}\right)$ (with respect to convolution), we can talk about objects in $\operatorname{Shv}_{\mathcal{G}_{q}^{\text {ratio }}}\left(Z^{-}\right)$being modules over this algebra.

The following result is a metaplectic extension of [BG2, Theorems 4.2 and 6.6]:

Theorem 6.7.2.

(a) There exists a canonically defined action of $\Omega^{\sharp, c l}$ on $\nabla_{q, Z}^{-}$.

(b) The map $\nabla_{q, Z}^{-} \rightarrow \mathrm{IC}_{q, Z}^{-}$induces an isomorphism

$$
\operatorname{Bar}\left(\Omega^{\sharp, c l}, \nabla_{q, Z}^{-}\right) \simeq \mathrm{IC}_{q, Z}^{-} .
$$

As a formal corollary, we obtain:

Corollary 6.7.3. There exists a canonically defined action of $\Omega^{\sharp, c l}$ on $\operatorname{Gauss}_{q, !}^{-}$so that the map

$$
\operatorname{Gauss}_{q, !}^{-} \rightarrow \operatorname{Gauss}_{q, ! *}^{-}
$$

induces an isomorphism

$$
\operatorname{Bar}\left(\Omega^{\sharp, c l}, \operatorname{Gauss}_{q, !}^{-}\right) \simeq \operatorname{Gauss}_{q, ! *}^{-} .
$$


6.7.4. Proof of Theorem 6.6.2. The proof is obtained by applying $\pi_{*}^{-}$to the isomorphism of Corollary 6.7.3. see Remark 4.4.4

Remark 6.7.5. As in BG2], Theorem 6.7.2 is closely related the following statement, which we have used in the proof of Lemma 6.3.4 and which is a metaplectic extension of [BFGM, Theorem 4.5] (a full proof in the metaplectic case is given in [Lys1, Theorem 4.1]):

Consider the !-restriction of $\mathrm{IC}_{q, Z}^{-}$

$$
\stackrel{\circ}{z} \simeq\{x\} \times \stackrel{\circ}{\iota^{\iota}} \stackrel{{ }_{\lambda} \times \mathrm{id}}{\longrightarrow} X^{\lambda} \times \stackrel{\circ}{z} \subset X^{\lambda} \times z^{-} \stackrel{\mathbf{i}^{\lambda}}{\longrightarrow} z^{-} .
$$

Then this restriction vanishes unless $\lambda \in \Lambda^{\sharp}$, and in the latter case identifies canonically with

$$
\underset{n>0}{\oplus} \operatorname{Sym}^{n}\left(\left(\mathfrak{n}^{\sharp}\right)^{\vee}\right)(\lambda)[-2 n] \otimes \mathrm{IC}_{q, \stackrel{\circ}{\check{z}}} .
$$

\section{Geometry and combinatorics of Theorem 6.3.8}

\subsection{The goal.}

7.1.1. With no restriction of generality, in this section we will assume that our root system is simple. Write

$$
q=\zeta \cdot q_{\mathbb{Z}}^{\min }
$$

where $q_{Z}^{\min }$ is the minimal integer-valued quadratic form on $\Lambda$ for $\zeta \in \mathfrak{Z}$.

We will assume that $q$ is non-degenerate. This means that $\operatorname{ord}(\zeta)$ is not divisible by

$$
d:=\frac{q_{\mathbb{Z}}^{\min }\left(\alpha_{l}\right)}{q_{\mathbb{Z}}^{\min }\left(\alpha_{s}\right)},
$$

where $\alpha_{l}$ and $\alpha_{s}$ are the long and the short roots, respectively.

In this section we will not be assuming that $q$ avoids small torsion.

We will analyze what Theorem 6.3.8 says in geometric terms for a given value of ord $(\zeta)$. We will show that its assertion is combinatorial in nature. Our concrete goal is to prove the following:

Theorem 7.1.2. The assertion of Theorem 6.3.8 for a given value of $\operatorname{ord}(\zeta)$ over a ground field of characteristic 0 implies the assertion for the same value of $\operatorname{ord}(\zeta)$ over any ground field.

7.1.3. Given that we have already proved Theorem 6.3.8 over a ground field of characteristic 0 (for $q$ that avoids small torsion), we obtain that Theorem 7.1.2 implies Theorem 6.3.8 over any ground field (also, for $q$ that avoids small torsion).

As we have seen in Sect. 6.3 this in turn implies Theorem 6.2.6 and further by Sect. 6.5 also Theorem 6.2.5

\subsection{Scrutinizing irreducible components.}

7.2.1. Recall that Theorem 6.3.8 says that (for $\lambda$ not a negative simple root) the cohomology

$$
H_{c}^{i}\left(S^{0} \cap S^{-, \lambda}, \Psi_{q, \lambda} \stackrel{*}{\otimes} \chi^{*}(\exp )\right)
$$

vanishes in (the sub-top) degree

$$
i=-\langle\lambda, 2 \check{\rho}\rangle-1 .
$$

Recall that $\operatorname{dim}\left(S^{0} \cap S^{-, \lambda}\right)=-\langle\lambda, \check{\rho}\rangle$. Hence, for (the top) degree $i=-\langle\lambda, 2 \check{\rho}\rangle$ and (the sub-top) degree $i=-\langle\lambda, 2 \check{\rho}\rangle-1$, the cohomology in (7.1) receives a surjective map from the direct sum of

$$
H_{c}^{i}\left(Z, \Psi_{q, \lambda} \stackrel{*}{\otimes} \chi^{*}(\exp )\right),
$$

where $Z$ runs over the set of the irreducible components of $S^{0} \cap S^{-, \lambda}$ of the (top) dimension $-\langle\lambda, \check{\rho}\rangle$. (Note, however, that $S^{0} \cap S^{-, \lambda}$ is known to be equidimensional.) 
We will now analyze which irreducible components $Z$ may have a potentially non-vanishing cohomology (7.2) in degree $-\langle\lambda, 2 \check{\rho}\rangle-1$.

Along the way we will see that this cohomology automatically vanishes in (the top) degree $-\langle\lambda, 2 \check{\rho}\rangle$ (for all $\lambda \in \Lambda^{\text {neg }}$ ).

7.2.2. Note that the map $\chi: S^{0} \rightarrow \mathbb{G}_{a}$ naturally factors as

$$
S^{0} \stackrel{\chi_{L}}{\rightarrow} \mathbb{G}_{a}^{I} \rightarrow \mathbb{G}_{a},
$$

where:

- $I$ is the set of vertices of the Dynkin diagram, and $\mathbb{G}_{a}^{I}$ is identified with $N /[N, N]$;

- $\mathbb{G}_{a}^{I} \rightarrow \mathbb{G}_{a}$ is the sum map.

The map $\chi_{I}$ is equivariant with respect to the $T$-action on $S^{0} \cap S^{-, \lambda}$ and the adjoint action of $T$ on $\mathbb{G}_{a}^{I} \simeq N /[N, N]$. Hence, for every irreducible component $Z$ of $S^{0} \cap S^{-, \lambda}$ there exists a subset $I_{Z} \subset I$, such that the map $\left.\chi\right|_{W}$ is a dominant map to $\mathbb{G}_{a}^{I_{Z}} \subset \mathbb{G}_{a}^{I}$.

Moreover, it follows from [FGV, Proposition 7.1.7] that as long as $\lambda$ is non-zero, $I_{Z} \neq \emptyset$.

7.2.3. We will consider two cases:

(i) $\left|I_{Z}\right| \geq 2$;

(ii) $I_{Z}$ is a singleton, i.e., $\{i\}$ for one vertex of the Dynkin diagram;

We will now show that in case (i), the sub-top cohomology of (7.2) vanishes. We declare an irreducible components in case (ii) as under scrutiny, and we will analyze them further in the next subsection.

7.2.4. We interpret

$$
\mathrm{C}_{c}\left(Z, \Psi_{q, \lambda} \stackrel{*}{\otimes} \chi^{*}(\exp )\right)
$$

as the ${ }^{*}$-fiber of the (un-normalized) Fourier transform of

$$
\left(\chi_{I} \mid z\right) !\left(\Psi_{q, \lambda}\right)
$$

at the point of the dual vector space of $\mathbb{G}_{a}^{I}$ corresponding to the sum map

$$
\mathbb{G}_{a}^{I} \rightarrow \mathbb{G}_{a}
$$

The object (7.3) in $\operatorname{Shv}\left(\mathbb{G}_{a}^{I}\right)$ is twisted $T$-equivariant. Hence so is its Fourier transform. In particular, this Fourier transform is lisse on the subset consisting of non-degenerate characters.

Since Fourier transform maps $\operatorname{Perv}\left(\mathbb{G}_{a}^{I}\right)$ to $\operatorname{Perv}\left(\mathbb{G}_{a}^{I}\right)[-|I|]$, it suffices to show that the object (7.3) lives in (perverse) cohomological degrees $\leq-\langle\lambda, 2 \breve{\rho}\rangle-1$, and that the inequality is strict if $\left|I_{Z}\right| \geq 2$.

This estimate would also imply that the top cohomology of (7.1) vanishes for all $\lambda \in \Lambda^{\text {neg }}-0$.

7.2.5. Again, due to the twisted $T$-equivariance, to prove the required cohomological estimate it suffices to show that the ${ }^{*}$-fiber of (7.3) at the generic point of each $\mathbb{A}^{I^{\prime}}$ (for a subset $I^{\prime} \subset I$ ) lives in degrees $\leq-\langle\lambda, 2 \check{\rho}\rangle-\left|I^{\prime}\right|-1$, and that the inequality is strict if $\left|I_{Z}\right| \geq 2$.

A priori, the ${ }^{*}$-fiber of $(7.3)$ at the generic point of $\mathbb{A}^{I^{\prime}}$ lives in degrees

$$
\leq 2(\operatorname{dim}(F)),
$$

where $F$ is the fiber of $\left.\chi_{I}\right|_{Z}$ over this point. Thus, we need to show that

$$
2(\operatorname{dim}(F)) \leq-\langle\lambda, 2 \check{\rho}\rangle-\left|I^{\prime}\right|-1,
$$

and the inequality is strict if $\left|I_{Z}\right| \geq 2$.

We have

$$
\operatorname{dim}(F) \leq \operatorname{dim}(Z)-\left|I^{\prime}\right|=-\langle\lambda, \check{\rho}\rangle-\left|I^{\prime}\right| .
$$

with the equality achieved only if $I^{\prime}=I_{Z}$. This implies the inequality in (7.4). 
7.3. The suspects. Let $Z$ be an irreducible component of the intersection $S^{0} \cap S^{-, \lambda}$, and let $j$ be a vertex of the Dynkin diagram. We will recall, following [BG3], a recipe that attaches to the pair $(Z, j)$ a non-negative integer $\phi_{j}(Z)$.

The main conclusion of this subsection will be that if $Z$ is an irreducible component of $S^{0} \cap S^{-, \lambda}$ under scrutiny with $I_{Z}=\{i\}$ and $\phi_{i}(Z) \geq 2$, then sub-the top cohomology in (7.2) still vanishes.

We will declare the irreducible components $Z$ under scrutiny for which $\phi_{i}(Z)=1$ as suspicious.

7.3.1. Let $P_{j} \subset G$ be the standard sub-minimal parabolic corresponding to $j$. Consider the corresponding diagram

Consider the ind-scheme

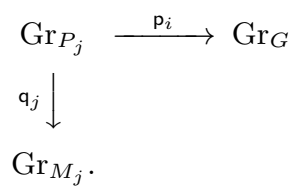

$$
\operatorname{Gr}_{P_{j}} \underset{\operatorname{Gr}_{G}}{\times} S^{-, \lambda}
$$

along with its projection to $\operatorname{Gr}_{M_{j}}$. Note that $\operatorname{Gr}_{P_{j}} \underset{\mathrm{Gr}_{G}}{\times} S^{-, \lambda}$ carries an action of $\mathfrak{L}\left(N_{j}^{-}\right)$, where $N_{j}^{-} \simeq \mathbb{G}_{a}$ is the (negative) maximal unipotent in $M_{j}$.

For a given $\mu \in \Lambda$, let $S_{j}^{-, \mu} \subset \mathrm{Gr}_{M_{j}}$ denote the corresponding orbit of $\mathfrak{L}\left(N_{j}^{-}\right)$. The above action defines an isomorphism

$$
\left(\mathbf{q}_{j}\right)^{-1}\left(S_{j}^{-, \mu}\right) \underset{\mathrm{Gr}_{G}}{\times} S^{-, \lambda} \simeq S_{j}^{-, \mu} \times\left(\left(\mathfrak{L}\left(N^{j}\right) \cdot t^{\mu}\right) \cap S^{-, \lambda}\right),
$$

where $N^{j}$ denotes the unipotent radical of $P_{j}$.

In terms of the isomorphism (7.5), the subset

$$
\left(\mathbf{q}_{j}\right)^{-1}\left(S_{j}^{-, \mu}\right) \underset{\mathrm{Gr}_{G}}{\times}\left(S^{0} \cap S^{-, \lambda}\right) \subset\left(\mathbf{q}_{j}\right)^{-1}\left(S_{j}^{-, \mu}\right) \underset{\mathrm{Gr}_{G}}{\times} S^{-, \lambda}
$$

corresponds to

$$
\left(S_{j}^{0} \cap S_{j}^{-, \mu}\right) \times\left(\left(\mathfrak{L}\left(N^{j}\right) \cdot t^{\mu}\right) \cap S^{-, \lambda}\right) .
$$

Thus, we obtain an isomorphism

$$
\left(\mathbf{q}_{j}\right)^{-1}\left(S_{j}^{-, \mu}\right) \underset{\operatorname{Gr}_{G}}{\times}\left(S^{0} \cap S^{-, \lambda}\right) \simeq\left(S_{j}^{0} \cap S_{j}^{-, \mu}\right) \times\left(\left(\mathfrak{L}\left(N^{j}\right) \cdot t^{\mu}\right) \cap S^{-, \lambda}\right) .
$$

7.3.2. Note that the intersection $S_{j}^{0} \cap S_{j}^{-, \mu}$ is taking place in neutral connected component of the affine Grassmannian of $\mathrm{Gr}_{M_{j}}$, which is a reductive group of semi-simple rank 1, so if the above intersection is non-empty, we have

$$
\mu=m \cdot\left(-\alpha_{j}\right)
$$

for a non-negative integer $m$.

Furthermore, we have:

$$
S_{j}^{0} \cap S_{j}^{-, \mu}=\left\{\begin{array}{l}
\text { pt if } m=0, \\
\mathbb{G}_{m} \text { if } m=1, \\
\mathbb{G}_{m} \times \mathbb{G}_{a}^{m-1} \text { if } m \geq 2 .
\end{array}\right.
$$

7.3.3. Let $Z$ be an irreducible component of $S^{0} \cap S^{-, \lambda}$ of dimension $-\langle\lambda, \check{\rho}\rangle$. One shows (see [BG3, Proposition 3.1]) that there exists a unique element $\mu \in \Lambda$, such that the intersection

$$
Z_{j}:=Z \cap\left(\mathbf{q}_{j}\right)^{-1}\left(S_{j}^{-, \mu}\right)
$$

is dense in $Z$.

We set $\phi_{j}(Z)$ to be the corresponding integer $m$ from (7.7). 
7.3.4. Let $\chi_{j}$ denote the composite

$$
S^{0} \stackrel{\chi_{I}}{\longrightarrow} \mathbb{G}_{a}^{I} \rightarrow \mathbb{G}_{a},
$$

where the last arrow is the projection on the $j$-th coordinate.

For an irreducible component $Z$, let $Z_{j}$ be as above. We obtain that the restriction $\left.\chi_{j}\right|_{Z_{j}}$ can be described as follows:

- It is the zero map if $\phi_{j}(Z)=0$;

- It is is the composite

$$
\begin{aligned}
& \quad Z_{j} \hookrightarrow\left(S_{j}^{0} \cap S_{j}^{-, \mu}\right) \times\left(\left(\mathfrak{L}\left(N^{j}\right) \cdot t^{\mu}\right) \cap S^{-, \lambda}\right) \rightarrow S_{j}^{0} \cap S_{j}^{-, \mu} \simeq \mathbb{G}_{m} \hookrightarrow \mathbb{G}_{a} \\
& \text { if } \phi_{j}(Z)=1 ;
\end{aligned}
$$

- It is is the composite

$$
Z_{j} \hookrightarrow\left(S_{j}^{0} \cap S_{j}^{-, \mu}\right) \times\left(\left(\mathfrak{L}\left(N^{j}\right) \cdot t^{\mu}\right) \cap S^{-, \lambda}\right) \rightarrow S_{j}^{0} \cap S_{j}^{-, \mu} \simeq \mathbb{G}_{m} \times \mathbb{G}_{a}^{m-1} \rightarrow \mathbb{G}_{a}^{m-1} \rightarrow \mathbb{G}_{a},
$$

where the last arrow is the projection on the first $\mathbb{G}_{a}$ factor.

7.3.5. Let $Z$ be a suspicious irreducible component of $S^{0} \cap S^{-, \lambda}$ and let $I_{Z}=\{i\}$. We obtain that $\phi_{j}(Z)=0$ for all $j \neq i$.

We will now show that if $\phi_{i}(Z) \geq 2$, then the sub-top cohomology in (17.2) still vanishes. Indeed, since $Z_{i}$ is dense in $Z$, it suffices to show that the sub-top cohomology in

$$
\mathrm{C}_{c}\left(Z_{i}, \Psi_{q, \lambda} \stackrel{*}{\otimes} \chi^{*}(\exp )\right)
$$

vanishes. However, we claim that (7.8) vanishes entirely.

Indeed, let us calculate (7.8) via the projection formula. We obtain that it identifies with

$$
\mathrm{C}_{c}\left(\mathbb{G}_{a},\left(\chi_{I} \mid z_{i}\right) !\left(\Psi_{q, \lambda}\right) \stackrel{*}{\otimes} \exp \right) .
$$

However, we claim that $\left(\chi_{I} \mid Z_{i}\right) !\left(\Psi_{q, \lambda}\right)$ is a constant complex on $\mathbb{G}_{a}$.

Indeed, in Sect. 7.5.3 we will see that the restriction of the local system $\Psi_{q, \lambda}$ to

$$
\left(\mathbf{q}_{j}\right)^{-1}\left(S_{j}^{-, \mu}\right) \underset{\mathrm{Gr}_{G}}{\times}\left(S^{0} \cap S^{-, \lambda}\right)
$$

is the pullback of a local system along the projection

$$
\begin{aligned}
\left(\mathbf{q}_{j}\right)^{-1}\left(S_{j}^{-, \mu}\right) \underset{\mathrm{Gr}_{G}}{\times}\left(S^{0} \cap S^{-, \lambda}\right) & \simeq\left(S_{j}^{0} \cap S_{j}^{-, \mu}\right) \times\left(\left(\mathfrak{L}\left(N^{j}\right) \cdot t^{\mu}\right) \cap S^{-, \lambda}\right) \simeq \\
& \simeq\left(\mathbb{G}_{m} \times \mathbb{G}_{a}^{m-1}\right) \times\left(\left(\mathfrak{L}\left(N^{j}\right) \cdot t^{\mu}\right) \cap S^{-, \lambda}\right) \rightarrow \mathbb{G}_{m} \times\left(\left(\mathfrak{L}\left(N^{j}\right) \cdot t^{\mu}\right) \cap S^{-, \lambda}\right) .
\end{aligned}
$$

This implies our assertion.

7.3.6. We declare an irreducible component $Z$ under scrutiny with $I_{Z}=\{i\}$ as suspicious if $\phi_{i}(Z)=1$. From what we have seen above, only suspicious irreducible components may contribute to the sub-top cohomology in (7.2).

7.3.7. Here is an example of a suspicious component: take $G=S L_{3}$ with the simple roots $\alpha$ and $\beta$. Take $\lambda=-\alpha-\beta$. Then both irreducible components of $S^{0} \cap S^{-, \lambda}$ are suspicious.

Remark 7.3.8. As is explained in Lys2, Prop. 1.2.4], for a given $G$ there are at most finitely many $\lambda$, such that $S^{0} \cap S^{-, \lambda}$ contains a suspicious irreducible component.

Namely, it is shown in loc.cit. that if $Z$ is such a component and $i$ is the corresponding vertex of the Dynkin diagram, then $\varpi_{i}+\lambda$ must appear as a weight in the irreducible $\check{G}$-representation with highest weight $\varpi_{i}$.

7.4. Interlude: coordinates on the irreducible components. In this subsection we will essentially reproduce a construction from $\mathrm{BaGa}$, Sect. 4]. 
7.4.1. Let $\mathcal{L}^{\text {min }}$ be the minimal line bundle on $\mathrm{Gr}_{G}$. It is $T$-equivariant by construction, with $Z_{G}$ acting trivially, so it is in fact $T_{\text {ad }}$-equivariant.

7.4.2. The action of $T_{\text {ad }}$ on the fiber of $\mathcal{L}^{\text {min }}$ at $t^{\lambda} \in \mathrm{Gr}_{G}$ is given by the character

$$
T_{\text {ad }} \rightarrow \mathbb{G}_{m}
$$

corresponding to

$$
b_{\mathbb{Z}}^{\min }(\lambda,-), \quad \Lambda_{\text {ad }} \rightarrow \mathbb{Z} .
$$

Here $b_{\mathbb{Z}}^{\min }$ is the integer-valued symmetric bilinear form on $\Lambda$, corresponding to the minimal quadratic form $q_{\mathbb{Z}}^{\min }$, and where we note that $b_{\mathbb{Z}}^{\min }$ extends to a pairing

$$
\Lambda \otimes \Lambda_{\mathrm{ad}} \rightarrow \mathbb{Z}
$$

by the formula

$$
b_{\mathbb{Z}}^{\min }\left(\alpha_{i}, \mu\right)=q_{\mathbb{Z}}^{\min }\left(\alpha_{i}\right) \cdot\left\langle\mu, \check{\alpha}_{i}\right\rangle .
$$

7.4.3. The restriction $\left.\mathcal{L}^{\text {min }}\right|_{S^{0}}$ admits a unique $\mathfrak{L}(N)$-equivariant trivialization, compactible with the trivialization of the fiber of $\mathcal{L}^{\text {min }}$ at $1 \in \mathrm{Gr}_{G}$. This trivialization is $T_{\text {ad-equivariant. }}$

Similarly, a choice of a trivialization of the fiber of $\mathcal{L}^{\text {min }}$ at $t^{\lambda} \in \operatorname{Gr}_{G}$ extends uniquely to an $\mathfrak{L}\left(N^{-}\right)$-equivariant trivialization of $\left.\mathcal{L}^{\mathrm{min}}\right|_{S^{-, \lambda}}$. This trivialization is twisted $T_{\text {ad }}$-equivariant against the character (7.9).

7.4.4. We obtain that the restriction $\left.\mathcal{L}^{\text {min }}\right|_{S^{0} \cap S^{-}, \lambda}$ admits two different trivializations (one is defined canonically, and another up to a multiplicative constant). Their discrepancy is a function

$$
f_{\lambda}: S^{0} \cap S^{-, \lambda} \rightarrow \mathbb{G}_{m},
$$

well-defined up to a multiplicative constant. This function in twisted $T_{\text {ad }}$-equivariant, against the character (7.9).

We will now show that irreducible components of the intersections $S^{0} \cap S^{-, \lambda}$ admit rational coordinates, such that the function (7.11) is given by monomials (products of powers of the coordinates), up to a multiplicative constant.

7.4.5. Consider the set

$$
\mathrm{B}(\lambda):=\underset{\lambda^{\prime}}{\sqcup} \mathrm{B}(\lambda)_{\lambda^{\prime}}
$$

where $\mathrm{B}(\lambda)_{\lambda^{\prime}}$ is the set if irreducible components of all the intersections

$$
S^{\lambda^{\prime}} \cap S^{-, \lambda} \text {. }
$$

We will now recall, following [BG3], the construction on the set $\mathrm{B}(\lambda)$ of a structure of Kashiwara's crystal:

First off, the functions $\phi_{j}$ are constructed by the recipe of Sect. 7.3.3 with $S^{0}$ replaced by a general $S^{\lambda^{\prime}}$. The functions $\epsilon_{j}$ are set to take value $\infty$.

The operators $f_{j}$ are defined as follows. Let $Z$ be an irreducible component of $S^{\lambda^{\prime}} \cap S^{-, \lambda}$ such that $\phi_{j}(Z) \neq 0$. Let $\mu$ be the corresponding element of $\Lambda$, see Sect. 7.3.3

Then, in terms of the identification

$$
Z_{j}:=\left(\mathbf{q}_{j}\right)^{-1}\left(S_{j}^{-, \mu}\right) \underset{\operatorname{Gr}_{G}}{\times}\left(S^{\lambda^{\prime}} \cap S^{-, \lambda}\right) \simeq\left(S_{j}^{\lambda^{\prime}} \cap S_{j}^{-, \mu}\right) \times\left(\left(\mathfrak{L}\left(N^{j}\right) \cdot t^{\mu}\right) \cap S^{-, \lambda}\right),
$$

$Z_{j}$ corresponds to a unique irreducible component $Z_{j}^{\prime} \subset\left(\mathfrak{L}\left(N^{j}\right) \cdot t^{\mu}\right) \cap S^{-, \lambda}$.

We let $f_{j}(Z)$ be the closure of the irreducible component

$$
\begin{aligned}
\left(S_{j}^{\lambda^{\prime}-\alpha_{j}} \cap S_{j}^{-, \mu}\right) \times Z_{j}^{\prime} \subset\left(S_{j}^{\lambda^{\prime}-\alpha_{j}} \cap S_{j}^{-, \mu}\right) \times & \left(\left(\mathfrak{L}\left(N^{j}\right) \cdot t^{\mu}\right) \cap S^{-, \lambda}\right) \simeq \\
& \simeq\left(\mathbf{q}_{j}\right)^{-1}\left(S_{j}^{-, \mu}\right) \underset{\operatorname{Gr}_{G}}{\times}\left(S^{\lambda^{\prime}-\alpha_{j}} \cap S^{-, \lambda}\right) \subset S^{\lambda^{\prime}-\alpha_{j}} \cap S^{-, \lambda}
\end{aligned}
$$


The operation $e_{j}$ is uniquely determined by the requirement that $f_{j} \circ e_{j}=\mathrm{id}$.

7.4.6. Let $Z$ be an irreducible component of some $S^{\lambda^{\prime}} \cap S^{-, \lambda}$. We will now use the crystal structure on $\mathrm{B}(\lambda)$ to introduce rational coordinates on $Z$.

Choose a string of vertices of the Dynkin diagram inductively as follows. If $\lambda^{\prime}=\lambda$, we have $Z=\mathrm{pt}$, and there is nothing to do. Otherwise, set $\lambda_{1}^{\prime}=\lambda^{\prime}, Z_{1}=Z$ and choose $j_{1} \in I$ such that $\phi_{j_{1}}\left(Z_{1}\right) \neq 0$. Set $Z_{2}=f_{j}^{\phi_{j_{1}}}\left(Z_{1}\right)$. Set

$$
\lambda_{2}^{\prime}=\lambda_{1}^{\prime}-\phi_{j_{1}}\left(Z_{1}\right) \cdot \alpha_{j_{1}}
$$

Now repeat the process with $Z_{1}$ replaced by $Z_{2}$. This process will terminate by (7.12) because

$$
S^{\lambda^{\prime}} \cap S^{-, \lambda} \neq \emptyset \Rightarrow \lambda^{\prime}-\lambda \in \Lambda^{\text {pos }}
$$

so for some $n$ we will have $\lambda_{n}^{\prime}=\lambda$.

Note that by construction, we have a rational isomorphism

$$
Z_{n} \simeq\left(\mathbb{G}_{m} \times \mathbb{G}_{a}^{\phi_{j_{n}}\left(Z_{n}\right)-1}\right) \times Z_{n+1} .
$$

The above process gives $Z$ rational coordinates. We will denote them by

$$
x_{j_{1}, 1}, \ldots, x_{j_{1}, \phi_{j_{1}}\left(Z_{1}\right)}, x_{j_{2}, 1}, \ldots, x_{j_{2}, \phi_{j_{2}}}\left(Z_{2}\right), \ldots
$$

7.4.7. Note that the construction of Sect. 7.4.4 defines a function (up to a multiplicative scalar) on each intersection $S^{\lambda^{\prime}} \cap S^{-, \lambda}$; let us denote it by $f_{\lambda^{\prime}, \lambda}$. We claim:

Proposition 7.4.8. The restriction of $f_{\lambda^{\prime}, \lambda}$ to a given irreducible component $Z$ equals (up to a multiplicative scalar) in terms of the coordinates (7.14) to

$$
\prod_{n}\left(x_{j_{n}, 1}\right)^{\phi_{j_{n}}\left(Z_{n}\right) \cdot q_{\mathbb{Z}}^{\min }\left(\alpha_{j_{n}}\right)} .
$$

Proof. It follows from the construction that in terms of the rational isomorphism (7.13), we have

$$
f_{\lambda_{n}^{\prime}, \lambda}=g_{n} \cdot f_{\lambda_{n+1}^{\prime}, \lambda}
$$

where $g_{n}$ is an invertible function on

$$
S_{j_{n}}^{\lambda_{n}^{\prime}} \cap S_{j_{n}}^{-, \lambda_{n}^{\prime}-\phi_{j_{n}}\left(Z_{n}\right) \cdot \alpha_{j_{n}}} \simeq \mathbb{G}_{m} \times \mathbb{G}_{a}^{\phi_{j_{n}}\left(Z_{n}\right)-1},
$$

equal to the restriction of $f_{\lambda_{n}^{\prime}, \lambda_{n}^{\prime}-\phi_{j_{n}}\left(Z_{n}\right) \cdot \alpha_{j_{n}}}$ along

$$
S_{j_{n}}^{\lambda_{n}^{\prime}} \cap S_{j_{n}}^{-, \lambda_{n}^{\prime}-\phi_{j_{n}}\left(Z_{n}\right) \cdot \alpha_{j_{n}}} \hookrightarrow S^{\lambda_{n}^{\prime}} \cap S^{-, \lambda_{n}^{\prime}-\phi_{j_{n}}\left(Z_{n}\right) \cdot \alpha_{j_{n}}} .
$$

Being invertible, the function $g_{n}$ equals, up to a multiplicative scalar, to the pullback of some power $m$ of the standard character on the $\mathbb{G}_{m}$ factor. Thus, it remains to show that the power in question equals $\phi_{j_{n}}\left(Z_{n}\right) \cdot q_{\mathbb{Z}}^{\min }\left(\alpha_{j_{n}}\right)$.

The function $x_{j_{n}, 1}$ is $\mathbb{G}_{m}$-equivariant against the character equal to $\check{\alpha}_{j_{n}}$.

The function $g_{n}$ is twisted $T_{\text {ad }}$-equivariant against the character equal to the ratio of the characters corresponding to $f_{\lambda_{n}^{\prime}, \lambda}$ and $f_{\lambda_{n+1}^{\prime}, \lambda}$, respectively. Hence, the character in question corresponds to the homomorphism

Hence, we obtain

$$
b_{\mathbb{Z}}^{\min }\left(\phi_{j_{n}}\left(Z_{n}\right) \cdot \alpha_{j_{n}},-\right): \Lambda_{\mathrm{ad}} \rightarrow \mathbb{G}_{m} .
$$

Evaluating on the fundamental coweight

$$
m \cdot \check{\alpha}_{j_{n}}=b_{\mathbb{Z}}^{\min }\left(\phi_{j_{n}}\left(Z_{n}\right) \cdot \alpha_{j_{n}},-\right) .
$$

$$
\varpi_{j_{n}}: \mathbb{G}_{m} \rightarrow T_{\mathrm{ad}}
$$

we obtain

$$
m=\phi_{j_{n}}\left(Z_{n}\right) \cdot b_{\mathbb{Z}}^{\min }\left(\alpha_{j_{n}}, \varpi_{j_{n}}\right)=\phi_{j_{n}}\left(Z_{n}\right) \cdot q_{\mathbb{Z}}^{\min }\left(\alpha_{j_{n}}\right) .
$$




\subsection{Indictment.}

7.5.1. Let $Z$ be a suspicious irreducible component. By the same analysis as in Sects. 7.2.4 7.2.5 we obtain that the sub-top cohomology in (7.2) (equivalently, in (7.8) ) is non-zero if and only if the restriction of the local system $\Psi_{q, \lambda}$ to $Z_{i}$ is (generically on $Z_{i}$ ) the pullback of a local system along the map

$$
\chi_{i}: Z_{i} \rightarrow \mathbb{G}_{m}
$$

We will call such $Z$ indicted. We will now analyze explicitly what it takes to be indicted.

7.5.2. Recall that our $q$ is written as

$$
\zeta \cdot q_{\mathbb{Z}}^{\min }
$$

where $q_{Z}^{\min }$ is the minimal quadratic form on $\Lambda$.

Note that the gerbe $\mathcal{G}_{q}^{G}$ over $\operatorname{Gr}_{G}$ identifies canonically with $\left(\mathcal{L}^{\mathrm{min}}\right)^{\zeta}$.

7.5.3. We obtain that the local system $\Psi_{q, \lambda}$ on $S^{0} \cap S^{-, \lambda}$ is the pullback of the Kummer local system $\Psi_{\zeta}$ on $\mathbb{G}_{m}$ by means of $f_{\lambda}$.

In particular, for a subscheme $Z \subset S^{0} \cap S^{-, \lambda}$, written as $Z \simeq Z^{\prime} \times \mathbb{G}_{a}$ with $Z^{\prime}$ reduced, the restriction of $\Psi_{q, \lambda}$ to $Z$ is the pullback from the $Z^{\prime}$ factor.

7.5.4. Let $Z$ be a suspicious component. Let us recall the (rational) coordinates on $Z$ constructed in Sect. 7.4.6 Note that we necessarily have $j_{1}=i$ and $\phi_{j_{1}}(Z)=\phi_{i}(Z)=1$. Note also that the map

$$
\left.\chi_{i}\right|_{Z_{i}}: Z_{i} \rightarrow \mathbb{G}_{m}
$$

identifies with the first coordinate function i.e., $x_{j_{1}, 1}$.

Hence, from Proposition 7.4.8 and Sect. 7.5.1 we obtain:

Corollary 7.5.5. A suspicious component $Z$ is indicted if and only if for all $n \geq 2$, the integers

$$
\phi_{j_{n}}\left(Z_{n}\right) \cdot q_{\mathbb{Z}}^{\min }\left(\alpha_{j_{n}}\right)
$$

are divisible by $\operatorname{ord}(\zeta)$.

Remark 7.5.6. One may wonder whether our indictment is non-empty: i.e., whether assuming that $q$ is non-degenerate indicted components exist. In fact, they do:

Take $G=G_{2}$; let $\alpha$ be the short simple root and let $\beta$ be the long simple root. Take $\lambda=-2 \alpha-\beta$. The intersection $S^{0} \cap S^{-, \lambda}$ has three irreducible components, among which exactly one is not annihilated by $e_{\beta}$.

Then this component is indicted for $\zeta=-1$.

Remark 7.5.7. Note that Corollary 7.5.5 implies that if $\zeta$ is non-torsion (i.e., $q$ is not a root of unity), then there are no indicted components.

Indeed, in this case $\operatorname{ord}(\zeta)=\infty$, so we would obtain that $\lambda$ is of the form $m \cdot\left(-\alpha_{i}\right)$, while the condition that $\phi_{i}(Z)=1$ forces $m=1$. I.e., we obtain that $\lambda$ is a negative simple root.

7.5.8. Thus, from now on we will assume that $\zeta$ is torsion (i.e., $q$ is a root of unity). We claim:

Corollary 7.5.9. Let $\lambda$ be such that $S^{0} \cap S^{-, \lambda}$ contains an indicted irreducible component $Z$; let $i$ be the corresponding element of $I$. Then:

(a) $\lambda+\alpha_{i} \in \Lambda^{\sharp}$.

(b) $\lambda \notin \Lambda^{\sharp}$. 
Proof. Let $Z$ be the indicted component. We have

$$
\lambda+\alpha_{i}=-\sum_{j \geq 2} \phi_{j_{n}}\left(Z_{n}\right) \cdot \alpha_{j_{n}} .
$$

Hence, to prove point (a), we need to show that

$$
\sum_{j \geq 2} \phi_{j_{n}}\left(Z_{n}\right) \cdot \alpha_{j_{n}} \in \Lambda^{\sharp}
$$

For this, it is sufficient to show that for every $j \geq 2$, the integer $\phi_{j_{n}}\left(Z_{n}\right)$ is divisible by ord $\left(q\left(\alpha_{j_{n}}\right)\right)$. However, this follows from Corollary 7.5.5

To prove point (b), it suffices to show that $\alpha_{i} \notin \Lambda^{\sharp}$. But this follows from the assumption that $q$ is non-degenerate.

Remark 7.5.10. The two conditions on $\lambda$, namely, that

$$
\lambda+\alpha_{i} \in \Lambda^{\sharp}
$$

and the condition from Remark 7.3.8, impose very stringent constraints.

The result of Lys2, Theorem 1.1.6] says that no indicted components exist, except for a very small number of possibilities for the order of $\zeta$.

7.6. Conviction. Let $Z$ be an indicted irreducible component of $S^{0} \cap S^{-, \lambda}$. This means that the cohomology

$$
H_{c}^{i}\left(Z, \Psi_{q, \lambda} \otimes \chi^{*}(\exp )\right),
$$

is non-zero in degree $i=-\langle\lambda, 2 \check{\rho}\rangle-1$.

In this subsection we will proceed to conviction: we will show that if $S^{0} \cap S^{-, \lambda}$ contains an indicted component $Z$, the cohomology

$$
H_{c}^{i}\left(S^{0} \cap S^{-, \lambda}, \Psi_{q, \lambda} \otimes \chi^{*}(\exp )\right)
$$

is also non-zero in degree $i=-\langle\lambda, 2 \check{\rho}\rangle-1$, in violation of Theorem 6.3 .8

7.6.1. First, we prove:

Proposition 7.6.2. Assume that $\lambda \notin \Lambda^{\sharp}$. Let $Z^{\prime} \subset S^{0} \cap S^{-, \lambda}$ be a closed $T$-stable subscheme of dimension $\leq-\langle\lambda, \check{\rho}\rangle-1$. Then the cohomology

$$
H_{c}^{i}\left(Z^{\prime}, \Psi_{q, \lambda} \otimes \chi^{*}(\exp )\right)
$$

vanishes in degrees $i \geq-\langle\lambda, 2 \check{\rho}\rangle-2$.

Proof. By the analysis in Sects 7.2.4 and 7.2.5. it suffices to consider the case when the the map $\chi_{I}$ sends $Z^{\prime}$ to $0 \in \mathbb{G}_{a}^{I}$. However, we claim that in this case

$$
\left(\left.\chi_{I}\right|_{Z^{\prime}}\right) !\left(\Psi_{q, \lambda}\right)=0 \text {. }
$$

Indeed, the assumption on $\lambda$ implies that the Kummer sheaf on $T$ given by (6.8) is non-trivial, while the point $0 \in \mathbb{G}_{a}^{I}$ does not support sheaves that are twisted $T$-equivariant against a non-trivial character sheaf.

Corollary 7.6.3. Assume that $\lambda \notin \Lambda^{\sharp}$. Then the map

$$
\underset{k}{\oplus} H_{c}^{i}\left(Z_{k}, \Psi_{q, \lambda} \otimes \chi^{*}(\exp )\right) \rightarrow H_{c}^{i}\left(S^{0} \cap S^{-, \lambda}, \Psi_{q, \lambda} \otimes \chi^{*}(\exp )\right)
$$

is injective for $i \geq-\langle\lambda, 2 \check{\rho}\rangle-1$, where the direct sum is taken over the set of irreducible components of $S^{0} \cap S^{-, \lambda}$ of dimension $-\langle\lambda, \check{\rho}\rangle$. 
7.6.4. Combining Corollaries 7.6.3 and 7.5.9 we obtain:

Corollary 7.6.5. Let $\lambda$ be such that $S^{0} \cap S^{-, \lambda}$ contains an indicted irreducible component. Then the cohomology (7.1) is non-zero in degree $-\langle\lambda, 2 \check{\rho}\rangle-1$.

The latter corollary says that the indicted components are indeed guilty of a crime: they bring about the failure of Theorem 6.3 .8

7.7. The verdict. We are finally ready to prove Theorem 7.1.2

Proof. Let us be working in both contexts simultaneously: $\ell$-adic sheaves over a field of positive characteristic or D-modules over a field of characterostic zero. In either case, write

$$
q=\zeta \cdot q_{\mathbb{Z}}^{\min }
$$

and choose $\zeta$ to be of the same order in both contexts.

By Corollary 7.6.5 it suffices to show the presence of an indicted components (for a given ord $(\zeta)$ ) is independent of which context we are working in.

However, by Corollary 7.5 .5 the existence of indicted components for a given root system is a property that can be expressed in terms of the the crystal $B(\lambda)$.

Hence, our assertion follows from Kashiwara's uniqueness theorem, which asserts that $B(\lambda)$ is uniquely recovered from the root system, and, in particular, it does not depend on the ground field.

\section{Appendix A. Sheaves with a formal parameter}

\section{A.1. Digression: sheaves with a formal parameter.}

A.1.1. For a fixed integer $n$ we can consider the sheaf theory, denoted $\operatorname{Shv}_{n}(-)$, obtained from our initial sheaf theory $\operatorname{Shv}(-)$ by tensoring with the ring e[ $[\hbar] / \hbar^{n}$, i.e., it sends a scheme $Y$ to

$$
\operatorname{Shv}_{n}(Y):=\left(\operatorname{Ind}(\operatorname{Shv}(Y)) \underset{\text { Vect }_{\mathrm{e}}}{\otimes} \mathrm{e}[\hbar] / \hbar^{n}-\bmod \right)^{c},
$$

where the superscript $c$ refers to the subcategory of compact objects.

Following GaLu, Sect. 2.3], define the sheaf theory $\mathrm{Shv}_{\llbracket \rrbracket}$ as the limit

$$
\operatorname{Shv}_{\llbracket \hbar \rrbracket}(Y):=\lim _{n} \operatorname{Shv}(Y)_{n},
$$

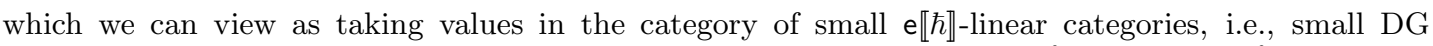
categories equipped with an action of the monoidal category $(e \llbracket \hbar \rrbracket-\bmod )^{\text {perf }}=(e \llbracket \hbar \rrbracket-\bmod )^{f \cdot g}$.

We have a tautological reduction $\bmod \hbar$ functor

$$
\mathcal{F} \mapsto \mathcal{F} \underset{e \llbracket \hbar \rrbracket}{\otimes} \mathrm{e}, \quad \operatorname{Shv}_{\llbracket \hbar \rrbracket}(Y) \rightarrow \operatorname{Shv}(Y) .
$$

A.1.2. Define the sheaf theory $\operatorname{Shv}_{((\hbar))}(-)$ to be the localization of $\operatorname{Shv}_{\llbracket \hbar \rrbracket}(-)$ with respect to $\hbar$, i.e.,

$$
\operatorname{Shv}_{((\hbar))}(Y):=\left(\operatorname{Ind}\left(\operatorname{Shv}_{\llbracket \hbar \rrbracket}(Y)\right) \underset{\mathrm{e} \llbracket \hbar \rrbracket-\bmod }{\otimes} \operatorname{Vect}_{\mathrm{e}((\hbar))}\right)^{c} .
$$

The tautological projection functor $\operatorname{Shv}_{\llbracket \hbar \rrbracket}(Y) \rightarrow \operatorname{Shv}_{((\hbar))}(Y)$ admits a right adjoint with values in the ind-completion $\operatorname{Ind}\left(\operatorname{Shv}_{\llbracket \hbar \rrbracket}(Y)\right)$ of $\operatorname{Shv}_{\llbracket \hbar \rrbracket}(Y)$. The composite functor

is the functor of $\hbar$-localization

$$
\operatorname{Shv}_{\llbracket \hbar \rrbracket}(Y) \rightarrow \operatorname{Shv}_{((\hbar))}(Y) \rightarrow \operatorname{Ind}_{\left(\operatorname{Shv}_{\llbracket \hbar \rrbracket}(Y)\right)}
$$

$$
\mathcal{F} \mapsto \operatorname{colim}(\mathcal{F} \stackrel{\hbar}{\rightarrow} \mathcal{F} \stackrel{\hbar}{\rightarrow} \ldots) .
$$

A.1.3. The standard functors (the !- and *- inverse and direct images) for $\operatorname{Shv}(-)$ induce the corresponding functors for $\operatorname{Shv}_{\llbracket \hbar \rrbracket}(-)$ by passage to the limit. Localizing with respect to $\hbar$, we obtain the corresponding functors for $\operatorname{Shv}_{((\hbar))}(-)$. 
D. GAITSGORY

A.2. The t-structure.

A.2.1. Repeating GaLu, Proposition 2.3.6.1], one proves:

Proposition A.2.2. For a scheme $Y$, the category $\operatorname{Shv}_{\llbracket \hbar \rrbracket}(Y)$ carries a t-structure uniquely characterized by the condition that an object $\mathcal{F} \in \operatorname{Shv}_{\llbracket \hbar \rrbracket}(Y)$ is connective, i.e., lies in $\left.\operatorname{Shv}_{\llbracket \hbar \rrbracket}(Y)\right)^{\leq 0}$, if and only if

$$
\mathcal{F} \underset{\mathrm{e} \llbracket \hbar \rrbracket}{\otimes} \mathrm{e} \in \operatorname{Shv}(Y)
$$

is connective (with respect to the perverse $t$-structure).

Remark A.2.3. The content of Proposition $\underline{A .2 .2(a)}$ is that for a given $\mathcal{F} \in \operatorname{Shv}_{\llbracket \hbar \rrbracket}(Y)$ there exists a fiber sequence

$$
\mathcal{F}_{1} \rightarrow \mathcal{F} \rightarrow \mathcal{F}_{2}
$$

with $\mathcal{F}_{1} \in\left(\operatorname{Shv}_{\llbracket \hbar \rrbracket}(Y)\right)^{\leq 0}$ (for the above definition of $\left.\left(\operatorname{Shv}_{\llbracket \hbar \rrbracket}(Y)\right)^{\leq 0}\right)$ and $\mathcal{F}_{2} \in\left(\left(\operatorname{Shv}_{\llbracket \hbar \rrbracket}(Y)\right)^{\leq 0}\right)^{\perp}$.

Remark A.2.4. The construction of the t-structure from Proposition A.2.2 is applicable in a more general context: we can start with any (small) DG category $\mathbf{C}$, equipped with a t-structure and such that $\mathbf{C}^{\odot}$ is Noetherian, and construct a t-structure on the corresponding category $\mathbf{C}_{\llbracket \hbar \rrbracket}$.

A.2.5. We will denote by $\operatorname{Perv}_{\llbracket \hbar \rrbracket}(Y)$ the heart of the t-structure on $\operatorname{Shv}_{\llbracket \hbar \rrbracket}(Y)$

Let us call an object of

$\hbar$-flat if

$$
\mathcal{F} \in \operatorname{Perv}_{\llbracket \hbar \rrbracket}(Y) \subset \operatorname{Shv}_{\llbracket \hbar \rrbracket}(Y)
$$

$$
\mathcal{F} \underset{\mathrm{e} \llbracket \hbar \rrbracket}{\otimes} \mathrm{e} \in \operatorname{Shv}(Y)
$$

lies in the heart of the t-structure, i.e., lies in $\operatorname{Perv}(Y)$.

For $\mathcal{F} \in \operatorname{Perv}_{\llbracket \hbar \rrbracket}(Y)$ denote

$$
\mathcal{F} / \hbar:=H^{0}(\mathcal{F} \underset{\mathrm{e} \llbracket \hbar \rrbracket}{\otimes} \mathrm{e}) .
$$

Note that if $\mathcal{F}$ is $\hbar$-flat, we have

$$
\mathcal{F} / \hbar \simeq \mathcal{F} \otimes_{\mathrm{e} \llbracket \hbar \rrbracket} \mathrm{e} .
$$

In general, $\mathcal{F} \otimes$ e may have a non-trivial $H^{-1}$, which is isomorphic to $\mathrm{e} \llbracket \hbar \rrbracket$

$$
\mathcal{F}[t]:=\operatorname{ker}(t: \mathcal{F} \rightarrow \mathcal{F}) .
$$

A.2.6. We claim:

Proposition A.2.7. The abelian category $\operatorname{Perv}_{\llbracket \hbar \rrbracket}(Y)$ is Noetherian, i.e., an increasing chain of subobjects in a given object stabilizes.

Proof. We will prove Proposition A.2.7 in the more general context of Remark A.2.4

For an object $\mathbf{c} \in\left(\mathbf{C}_{\llbracket \rrbracket \rrbracket}\right)^{\bigcirc}$ denote

$$
\mathbf{c}\left[\hbar^{d}\right]:=\operatorname{ker}\left(\hbar^{d}: \mathbf{c} \rightarrow \mathbf{c}\right) \in\left(\mathbf{C}_{\llbracket \hbar \rrbracket}\right)^{\odot}
$$

and

$$
\hbar^{d} \mathbf{c}:=\operatorname{Im}\left(\hbar^{d}: \mathbf{c} \rightarrow \mathbf{c}\right) \in\left(\mathbf{C}_{\llbracket \hbar \rrbracket}\right)^{\bigodot} .
$$

Lemma A.2.8. The sequence of subobjects

$$
d \mapsto \mathbf{c}\left[\hbar^{d}\right] \subset \mathbf{c}
$$

stabilizes. 
Let us assume this lemma for a moment and proceed with the proof of Proposition A.2.7

Let $\mathbf{c}\left[\hbar^{\infty}\right]$ denote the subobject of $\mathbf{c}$ equal to the eventual value of $\mathbf{c}\left[\hbar^{d}\right]$.

For a subobject $\mathbf{c}_{1} \subset \mathbf{c}$ we define its saturation $\widetilde{\mathbf{c}}_{1}$ to be the preimage of $\left(\mathbf{c} / \mathbf{c}_{1}\right)\left[\hbar^{\infty}\right]$ under the projection

$$
\mathbf{c} \rightarrow \mathbf{c} / \mathbf{c}_{1}
$$

Let

$$
\mathbf{c}_{1} \subset \mathbf{c}_{2} \subset \ldots \subset \mathbf{c}
$$

be a sequence of subobjects. Consider the corresponding sequence of their saturations

$$
\widetilde{\mathbf{c}}_{1} \subset \widetilde{\mathbf{c}}_{2} \ldots \subset \mathbf{c} .
$$

By construction, the corresponding maps

$$
\widetilde{\mathbf{c}}_{i} / \hbar \widetilde{\mathbf{c}}_{i} \rightarrow \mathbf{c} / \hbar \mathbf{c}
$$

are injective.

It is easy to see that as soon as

$$
\widetilde{\mathbf{c}}_{i} / \hbar \widetilde{\mathbf{c}}_{i} \hookrightarrow \widetilde{\mathbf{c}}_{i+1} / \hbar \widetilde{\mathbf{c}}_{i+1}
$$

is an isomorphism (which happens for some $i$ due to the Noetherianness of $\mathbf{C}^{\odot}$ ), we have $\widetilde{\mathbf{c}}_{i}=\widetilde{\mathbf{c}}_{i+1}$.

Replacing the initial $\mathbf{c}$ by the eventual value of $\widetilde{\mathbf{c}}_{i}$ and reindexing, we can assume that all $\widetilde{\mathbf{c}}_{i}=\mathbf{c}$. Taking the quotient, we can assume that $\mathbf{c}$ is torsion. Then the assertion follows from Lemma A.2.8

Proof of Lemma A.2.8 (due to J. Lurie). Multiplication by $\hbar^{n}$ defines surjective maps

$$
\mathbf{c} / \hbar \mathbf{c} \rightarrow \hbar^{n} \mathbf{c} / \hbar^{n+1} \mathbf{c} .
$$

By Noetherianness, the kernels of these maps stabilize. Hence, for some $n_{0}$ and all $n \geq n_{0}$, the maps

$$
\hbar^{n} \mathbf{c} / \hbar^{n+1} \mathbf{c} \stackrel{\hbar}{\rightarrow} \hbar^{n+1} \mathbf{c} / \hbar^{n+2} \mathbf{c}
$$

are isomorphisms.

By $\hbar$-completeness, the maps

$$
\hbar^{n} \mathbf{c} \stackrel{\hbar}{\rightarrow} \hbar^{n+1} \mathbf{c}
$$

are also isomorphisms. Hence, $\hbar$ is torsion-free on $\hbar^{n} \mathbf{c}$.

Hence, for any $d$,

$$
\mathbf{c}\left[\hbar^{d}\right] \hookrightarrow\left(\mathbf{c} / \hbar^{n} \mathbf{c}\right)\left[\hbar^{d}\right]
$$

Now, the assertion follows by Noetherianness.

A.2.9. We define a t-structure on $\operatorname{Shv}_{((\hbar))}(Y)$ to be uniquely characterized by the property that the localization functor

$$
\operatorname{Shv}_{\llbracket \hbar \rrbracket}(Y) \rightarrow \operatorname{Shv}_{((\hbar))}(Y)
$$

is t-exact.

The t-structure on $\operatorname{Shv}_{\llbracket \hbar \rrbracket}(Y)$ induces one on $\operatorname{Ind}\left(\operatorname{Shv}_{\llbracket \hbar \rrbracket}(Y)\right)$. The functor

$$
\operatorname{Shv}_{((\hbar))}(Y) \rightarrow \operatorname{Ind}\left(\operatorname{Shv}_{\llbracket \hbar \rrbracket}(Y)\right)
$$

right adjoint to the projection is also t-exact.

\section{A.3. Gerbes with a formal parameter.}


D. GAITSGORY

A.3.1. For a fixed $n$, one can talk about $\left(\mathrm{e}[\hbar] / \hbar^{n}\right)^{\times}$-gerbes on a given scheme/prestack $Y$, defined as in Sect. 0.5.6 Denote this category by $\operatorname{Ge}_{n}(Y)$. Define the category $\mathrm{Ge}_{\hbar}(Y)$ by

$$
\operatorname{Ge}_{\hbar}(Y):=\lim _{n} \operatorname{Ge}_{n}(Y)
$$

Given an object $\mathcal{G} \in \mathrm{Ge}_{\hbar}(Y)$, one can consider the category

$$
\operatorname{Shv}_{\mathcal{G}, \llbracket \hbar \rrbracket}(y)
$$

and its localization $\operatorname{Shv}_{\mathcal{G},((\hbar))}(y)$.

A.3.2. Define the group $\mathfrak{Z}_{n}$ in each of the sheaf theories from Sect. 0.5.4 as follows:

- For $\operatorname{Shv}(-)$ being constructible sheaves in classical topology with e-coefficients, set

$$
\mathfrak{Z}_{n}=\left(\mathrm{e}[\hbar] / \hbar^{n}\right)^{\times}
$$

- For $\operatorname{Shv}(-)$ being constructible $\ell$-adic sheaves, set $\mathfrak{Z}_{n}=\left(\overline{\mathbb{Z}}_{\ell}[\hbar] / \hbar^{n}\right)^{\times}$;

- For $\operatorname{Shv}(-)$ being holonomic D-modules, set $\mathfrak{Z}_{n}=\left(k[\hbar] / \hbar^{n}\right) / \mathbb{Z}$.

In all of these cases, an element $\zeta \in \mathfrak{Z}_{n}$ gives rise to a Kummer character sheaf $\Psi_{\zeta} \in \operatorname{Shv}_{n}\left(\mathbb{G}_{m}\right)$. Hence, for a line bundle $\mathcal{L}$ on $Y$ and $\zeta$ as above, we obtain a well-defined object

$$
\mathcal{L}^{\zeta} \in \mathrm{Ge}_{n}(Y) \text {. }
$$

A.3.3. Define

$$
\mathfrak{Z}_{\hbar}:=\lim _{n} \mathfrak{Z}_{n}
$$

We obtain that for a line bundle $\mathcal{L}$ on $Y$ and $\zeta \in \mathfrak{Z}_{\hbar}$, we obtain a well-defined object

$$
\mathcal{L}^{\zeta} \in \mathrm{Ge}_{\hbar}(Y) \text {. }
$$

\section{REFERENCES}

[BFGM] A. Braverman, M. Finkelberg, D. Gaitsgory and I. Mirkovic, Intersection cohomology of Drinfeld compactifications, Selecta Mathematica N.S. 8 (2002), 381-418.

[BFS] R. Bezrukavnikov, M. Finkelberg, V. Schechtman, Factorizable sheaves and quantum groups, Lecture Notes in Mathematics 1691 (1998).

[BaGa] P. Baumann and S. Gaussent, On Mirkovic-Vilonen cycles and crystals combinatorics, Jour. of Rep. Theory 12 (2008), 83-130.

[BG1] A. Braverman and D. Gaitsgory, Geometric Eisenstein series, Invent. Math. 150 (2002), 287-84.

[BG2] A. Braverman and D. Gaitsgory, Deformations of local system and Eisenstein series, GAFA 17 (2008), 1788-1850.

[BG3] A. Braverman and D. Gaitsgory, Crystals via the affine Gassmannian, Duke Math. Journal 107 (2001), 561-575.

[Camp] J. Campbell, A resolution of singularities for Drinfeld's compactification via stable maps, Journal of Alg. Geom. 28 (2019), 153-167.

[CD] J. Campbell and G. Dhillon, The tamely ramified Fundamental Local Equivalence at integral level, arXiv:1907.03204

[FGV] E. Frenkel, D. Gaitsgory and K. Vilonen, Whittaker patterns in the geometry of moduli spaces of bundles on curves, Annals of Math. 153 (2001), no. 3, 699-748.

[Ga1] D. Gaitsgory, The semi-infinite intersection cohomology sheaf, Adv. in Math. 327 (2018), 789-868. Also: arXiv: 1703.04199.

[Ga2] D. Gaitsgory, The semi-infinite intersection cohomology sheaf-II: the Ran space version, arXiv:1708.07205

[Ga3] D. Gaitsgory, Twisted Whittaker category and factorizable sheaves, Sel. Math., New ser. 13 (2008), 617-659.

[GaLu] D. Gaitsgory and J. Lurie, Weil's conjecture for function fields-1, Annals of Mathematical Studies 360, Princeton University Press (2018).

[GLys1] D. Gaitsgory and S. Lysenko, Parameters and duality for the metaplectic geometric Langlands theory, Selecta Math. New Ser. 24 (2018), 227-301. Also arXiv: 1608.00284

[GLys2] D. Gaitsgory and S. Lysenko, Metaplectic Whittaker category and quantum groups : the "small" FLE, arXiv: 1903.02279

[Geo] University of Georgia VIGRE algebra group, An analog of Kostant's theorem for the cohomology of quantum groups, Proc. AMS 138 (2010), 85-99.

[Lus] G. Lusztig, Introduction to quantum groups, Progress in Mathematics 110, Birkhäuser Boston (1993).

[Lys1] S. Lysenko, Geometric Eisenstein series: twisted setting, J. of the EMS 19 (2014), 3179-3252.

[Lys2] S. Lysenko, Twisted Whittaker models for metaplectic groups, GAFA 27 (2017), 289-372.

[Ras] S. Raskin, Chiral principal series categories I: finite-dimensional calculations, available from http://math.mit.edu/ sraskin/

[Ro] M. Rosso, Certaines formes bilinéaires sur les groupes quantiques et une conjecture de Schechtman et Varchenko, C. R. Acad. Sci. Paris Sér. I Math. 314 (1992), no. 1, 5-8. 